\title{
Design of cobalt Fischer-Tropsch catalysts for the combined production of liquid fuels and olefin chemicals from hydrogen-rich syngas
}

Kai Jeske ${ }^{1}$ Ali Can Kizilkaya ${ }^{2}$, Iván López-Luque ${ }^{3}$, Norbert Pfänder ${ }^{4}$, Matthias Bartsch, ${ }^{5}$ Patricia Concepción ${ }^{3}$, Gonzalo Prieto1,3,*

${ }^{1}$ Max-Planck-Institut für Kohlenforschung, Kaiser-Wilhelm-Platz 1, 45470 Mülheim an der Ruhr, Germany.

2 Department of Chemical Engineering, Izmir Institute of Technology, Gülbahçe Kampüsü, 35430 Izmir, Turkey.

${ }^{3}$ ITQ Instituto de Tecnología Química, Universitat Politècnica de València-Consejo Superior de Investigaciones Científicas (UPV-CSIC), Avenida de los Naranjos s/n, 46022 Valencia, Spain.

${ }^{4}$ Max-Planck-Institut für chemische Energiekonversion, Stiftstraße, 45470 Mülheim an der Ruhr, Germany.

${ }^{5}$ Faculty of Physics and CENIDE, Universität Duisburg-Essen, 47048 Duisburg, Germany.

*Email: prieto@mpi-muelheim.mpg.de; prieto@itq.upv.es

6. Structure files (DFT calculations)

i) Absolute energies of the structures reported in the manuscript (eV)

\begin{tabular}{|c|c|}
\hline 1) $\mathrm{PrO}_{2}-\mathrm{Co}(111)-2 \times 2$ & -128.89302516 \\
\hline 2) $\mathrm{PrO}_{2}-\mathrm{Co}(211)-1 \times 2$ & -238.57085732 \\
\hline 3) $\mathrm{Na}_{2} \mathrm{O}-\mathrm{Co}(111)-2 \times 2$ & -117.56242049 \\
\hline 4) $\mathrm{Na}_{2} \mathrm{O}-\mathrm{Co}(211)-1 \times 2$ & -227.52687570 \\
\hline 5) $\mathrm{PrO}_{2}-\mathrm{Co}(111)-3 \times 3$ & -262.49976992 \\
\hline 6) $\mathrm{PrO}_{2}-\mathrm{Co}(211)-1 \times 3$ & -346.22925763 \\
\hline 7) $\mathrm{Na}_{2} \mathrm{O}-\mathrm{Co}(111)-3 \times 3$ & -252.23074347 \\
\hline 8) $\mathrm{Na}_{2} \mathrm{O}-\mathrm{Co}(211)-1 \times 3$ & -335.67823594 \\
\hline 9) $\mathrm{H}-\mathrm{Co}(111)-3 \times 3$ & -244.23380844 \\
\hline 10) $\mathrm{H}-\mathrm{PrO}_{2}-\mathrm{Co}(111)-3 \times 3$ & -266.33876573 \\
\hline 11) $\mathrm{H}-\mathrm{Na}_{2} \mathrm{O}-\mathrm{Co}(111)-3 \times 3$ & -255.99209598 \\
\hline
\end{tabular}




\begin{tabular}{|c|c|}
\hline 12) $\mathrm{CO}-\mathrm{Co}(111)-3 \times 3$ & -256.82825463 \\
\hline 13) $\mathrm{CO}-\mathrm{PrO}_{2}-\mathrm{Co}(111)-3 \times 3$ & -279.67890552 \\
\hline 14) $\mathrm{CO}-\mathrm{Na}_{2} \mathrm{O}-\mathrm{Co}(111)-3 \times 3$ & -269.35040455 \\
\hline 15) $\mathrm{H}-\mathrm{Co}(211)-1 \times 3$ & -326.93677101 \\
\hline 16) $\mathrm{H}-\mathrm{PrO}_{2}-\mathrm{Co}(211)-1 \times 3$ & -350.05488436 \\
\hline 17) $\mathrm{H}-\mathrm{Na}_{2} \mathrm{O}-\mathrm{Co}(211)-1 \times 3$ & -339.52004017 \\
\hline 18) $\mathrm{CO}-\mathrm{Co}(211)-1 \times 3$ & -339.56825882 \\
\hline 19) $\mathrm{CO}-\mathrm{PrO}_{2}-\mathrm{Co}(211)-1 \times 3$ & -363.32903626 \\
\hline 20) $\mathrm{CO}-\mathrm{Na}_{2} \mathrm{O}-\mathrm{Co}(211)-1 \times 3$ & -352.67612255 \\
\hline 21) $\mathrm{C}_{2} \mathrm{H}_{4}-\mathrm{Co}(111)-3 \times 3$ & -272.86550325 \\
\hline 22) $\mathrm{C}_{2} \mathrm{H}_{4}-\mathrm{PrO}_{2}-\mathrm{Co}(111)-3 \times 3$ & -294.62661654 \\
\hline 23) $\mathrm{C}_{2} \mathrm{H}_{4}-\mathrm{Co}(211)-1 \times 3$ & -356.23248497 \\
\hline 24) $\mathrm{C}_{2} \mathrm{H}_{4}-\mathrm{PrO}_{2}-\mathrm{Co}(211)-1 \times 3$ & -378.9933074 \\
\hline 25) C-Co(111)-3x3 & -248.51457208 \\
\hline 26) $\mathrm{C}-\mathrm{PrO}_{2}-\mathrm{Co}(111)-3 \times 3$ & -270.88401842 \\
\hline 27) $\mathrm{C}-\mathrm{Na}_{2} \mathrm{O}-\mathrm{Co}(111)-3 \times 3$ & -260.63216452 \\
\hline 28) C-Co(211)-1x3 & -331.33418986 \\
\hline 29) $\mathrm{C}-\mathrm{PrO}_{2}-\mathrm{Co}(211)-1 \times 3$ & -354.63076785 \\
\hline 30) $\mathrm{C}-\mathrm{Na}_{2} \mathrm{O}-\mathrm{Co}(211)-1 \times 3$ & -344.1383606 \\
\hline
\end{tabular}

\section{ii) VASP POSCAR files}

1) $\mathrm{PrO}_{2}-\mathrm{Co}(111)-2 \times 2$

Co $\operatorname{Pr} \mathrm{O}$

1.00000000000000

$\begin{array}{llll}4.9752000000000001 & 0.0000000000000000 & 0.0000000000000000\end{array}$

$\begin{array}{llll}-2.4876000000000000 & 4.3086495888999998 & 0.0000000000000000\end{array}$

$\begin{array}{llll}0.0000000000000000 & 0.0000000000000000 & 21.0933999999999990\end{array}$

Co $\operatorname{Pr} \mathrm{O}$

$\begin{array}{lll}16 & 1 & 2\end{array}$

Selective dynamics

Direct

$\begin{array}{llllllll}0.4445037044393629 & 0.4722398417457470 & 0.0024679757649295 & F & F & F\end{array}$ $\begin{array}{llllllllll}0.9445037044393629 & 0.4722398417457470 & 0.0024679757649295 & F & F & F\end{array}$ $\begin{array}{lllllll}0.4445037282925455 & 0.9722398894521049 & 0.0024679757649295 & F & F & F\end{array}$ $\begin{array}{llllllll}0.9445037282925455 & 0.9722398894521049 & 0.0024679757649295 & F & F & F\end{array}$ $\begin{array}{llllllll}0.1114513153999539 & 0.3057535714656083 & 0.0948550731508462 & F & F & F\end{array}$ $\begin{array}{llllllll}0.6114513153999539 & 0.3057535714656083 & 0.0948550731508462 & F & F & F\end{array}$ $\begin{array}{lllllllll}0.1114513392531364 & 0.8057536191719734 & 0.0948550731508462 & F & F & F\end{array}$ $\begin{array}{lllllllll}0.6114513392531364 & 0.8057536191719734 & 0.0948550731508462 & F & F & F\end{array}$ 
$\begin{array}{lllllll}0.2731032588298067 & 0.1388428258455599 & 0.1928027940928242 & \mathrm{~T} & \mathrm{~T} & \mathrm{~T}\end{array}$ $\begin{array}{llllllllll}0.7775714905794112 & 0.1383873554515214 & 0.1934177543917787 & \mathrm{~T} & \mathrm{~T} & \mathrm{~T}\end{array}$ $\begin{array}{lllllllll}0.2746379882948871 & 0.6356880804870526 & 0.1937469262252913 & \mathrm{~T} & \mathrm{~T} & \mathrm{~T}\end{array}$ $\begin{array}{llllllllll}0.7744995809387224 & 0.6377803447482050 & 0.1948657948326394 & \mathrm{~T} & \mathrm{~T} & \mathrm{~T}\end{array}$ $\begin{array}{lllllllll}0.4455856674148032 & 0.4781918645404295 & 0.2898307587559638 & \mathrm{~T} & \mathrm{~T} & \mathrm{~T}\end{array}$ $\begin{array}{llllllll}0.9412495153114611 & 0.4687342373096127 & 0.2865710806432946 & \mathrm{~T} & \mathrm{~T} & \mathrm{~T}\end{array}$ $\begin{array}{llllllll}0.4452219384921141 & 0.9698445726518042 & 0.2839912253299769 & \mathrm{~T} & \mathrm{~T} & \mathrm{~T}\end{array}$ $\begin{array}{llllllll}0.9317520968679313 & 0.9641852533621050 & 0.2899777763113320 & \mathrm{~T} & \mathrm{~T} & \mathrm{~T}\end{array}$ $\begin{array}{llllllll}0.4084913512254580 & 0.8451342412281843 & 0.4189876214004703 & \mathrm{~T} & \mathrm{~T} & \mathrm{~T}\end{array}$ $\begin{array}{llllllll}0.2807349002319417 & 0.1328592794712665 & 0.3490955823335268 & \mathrm{~T} & \mathrm{~T} & \mathrm{~T}\end{array}$ $\begin{array}{lllllllllll}0.9516422081300646 & 0.4949668301481068 & 0.4390906674975716 & \mathrm{~T} & \mathrm{~T} & \mathrm{~T}\end{array}$

2) $\mathrm{PrO}_{2}-\mathrm{Co}(211)-1 \times 2$

Co $\operatorname{Pr} \mathrm{O}$

1.00000000000000

$\begin{array}{llll}6.0933999999999999 & 0.0000000000000000 & 0.0000000000000000\end{array}$

$\begin{array}{llll}0.0000000000000000 & 4.9752000000000001 & 0.0000000000000000\end{array}$

$\begin{array}{llll}0.0000000000000000 & 0.0000000000000000 & 25.7715999999999994\end{array}$

Co $\operatorname{Pr} \mathrm{O}$

$\begin{array}{lll}32 & 1 & 2\end{array}$

Selective dynamics

Direct

$\begin{array}{lllllll}0.3012132799999989 & 0.0000000000000000 & 0.0046948579056050 & \mathrm{~F} & \mathrm{~F} & \mathrm{~F}\end{array}$

$\begin{array}{llllllll}0.3012132799999989 & 0.5000000000000000 & 0.0046948579056050 & F & F & F\end{array}$

$\begin{array}{lllllll}0.6286634719999995 & 0.2500000000000000 & 0.0308760418445075 & F & F & F\end{array}$

$\begin{array}{lllllllll}0.6286634719999995 & 0.7500000000000000 & 0.0308760418445075 & F & F & F\end{array}$

$\begin{array}{lllllllllllll}0.9633304890000005 & 0.0000000000000000 & 0.0539849291468144 & F & F & F\end{array}$

$\begin{array}{lllllllllll}0.9633304890000005 & 0.5000000000000000 & 0.0539849291468144 & F & F & F\end{array}$

$\begin{array}{llllllllll}0.2960606560000016 & 0.2500000000000000 & 0.0837748529388946 & F & F & F\end{array}$

$\begin{array}{llllllll}0.2960606560000016 & 0.7500000000000000 & 0.0837748529388946 & F & F & F\end{array}$

$\begin{array}{llllllll}0.6289869370000005 & 0.0000000000000000 & 0.1118942944947179 & F & F & F\end{array}$

$\begin{array}{llllllll}0.6289869370000005 & 0.5000000000000000 & 0.1118942944947179 & F & F & F\end{array}$ $\begin{array}{llllllllll}0.9626366230000016 & 0.2500000000000000 & 0.1384130593366351 & F & F & F\end{array}$ $\begin{array}{lllllllll}0.9626366230000016 & 0.7500000000000000 & 0.1384130593366351 & F & F & F\end{array}$ $\begin{array}{lllllllll}0.2974300059999990 & 0.0000000000000000 & 0.1677073600397350 & F & F & F\end{array}$ $\begin{array}{llllllll}0.2974300059999990 & 0.5000000000000000 & 0.1677073600397350 & F & F & F\end{array}$ $\begin{array}{lllllll}0.6301967699999977 & 0.2500000000000000 & 0.1957913361995409 & F & F & F\end{array}$ $\begin{array}{llllllll}0.6301967699999977 & 0.7500000000000000 & 0.1957913361995409 & F & F & F\end{array}$ $\begin{array}{llllllll}0.9612393345043201 & 0.0001654511557178 & 0.2217530779742082 & \text { T } & \text { T } & \text { T }\end{array}$ $\begin{array}{llllllllll}0.9619602252223010 & 0.5007080034786174 & 0.2223508555645184 & \mathrm{~T} & \mathrm{~T} & \mathrm{~T}\end{array}$ $\begin{array}{llllllllll}0.2932882650735641 & 0.2509469490134886 & 0.2503303216932884 & \mathrm{~T} & \mathrm{~T} & \mathrm{~T}\end{array}$ $\begin{array}{llllllll}0.2952132657754088 & 0.7518241800958301 & 0.2508991737638424 & \mathrm{~T} & \mathrm{~T} & \mathrm{~T}\end{array}$ $\begin{array}{llllllll}0.6274213143218794 & 0.0033536120609225 & 0.2791209538839414 & \mathrm{~T} & \mathrm{~T} & \mathrm{~T}\end{array}$ $\begin{array}{llllllll}0.6295366930833761 & 0.4967964024061680 & 0.2797256661253752 & \mathrm{~T} & \mathrm{~T} & \mathrm{~T}\end{array}$ $\begin{array}{lllllllll}0.9619202854679206 & 0.2459953049042675 & 0.3048451383238431 & \mathrm{~T} & \mathrm{~T} & \mathrm{~T}\end{array}$ $\begin{array}{llllllllll}0.9626914900602375 & 0.7534252876280481 & 0.3061211429073926 & \mathrm{~T} & \mathrm{~T} & \mathrm{~T}\end{array}$ $\begin{array}{lllllllll}0.2949105437012648 & 0.0047520241691534 & 0.3350009482826016 & \mathrm{~T} & \mathrm{~T} & \mathrm{~T}\end{array}$ $\begin{array}{llllllllll}0.2976579892100570 & 0.4967995017534598 & 0.3346541804789939 & \text { T } & \text { T } & \text { T }\end{array}$ $\begin{array}{llllllll}0.6192171601653956 & 0.2452566944705538 & 0.3676970544897814 & \mathrm{~T} & \mathrm{~T} & \mathrm{~T}\end{array}$ $\begin{array}{lllllll}0.6224272945515560 & 0.7539016396365075 & 0.3595286477993909 & \mathrm{~T} & \mathrm{~T} & \mathrm{~T}\end{array}$ $\begin{array}{lllllllll}0.9653321004684635 & 0.0031621961181977 & 0.3895328192885472 & \mathrm{~T} & \mathrm{~T} & \mathrm{~T}\end{array}$ $\begin{array}{lllllllll}0.9644658444022296 & 0.4975548052334668 & 0.3838548951817233 & \mathrm{~T} & \mathrm{~T} & \mathrm{~T}\end{array}$ $\begin{array}{llllllllll}0.2915578224101827 & 0.2605495254074769 & 0.4194275015122335 & \mathrm{~T} & \mathrm{~T} & \mathrm{~T}\end{array}$ 
$\begin{array}{lllllll}0.2763778939310634 & 0.7568547364505791 & 0.4150143802033285 & \mathrm{~T} & \mathrm{~T} & \mathrm{~T}\end{array}$ $\begin{array}{lllllll}0.7026291092202090 & 0.6726542584438787 & 0.4674838650212905 & \mathrm{~T} & \mathrm{~T} & \mathrm{~T}\end{array}$ $\begin{array}{lllllll}0.8059941344833528 & 0.2513628050176295 & 0.4376669279132808 & \mathrm{~T} & \mathrm{~T} & \mathrm{~T}\end{array}$ $\begin{array}{llllllll}0.3552186555693522 & 0.5245467981836430 & 0.4712444666277791 & \mathrm{~T} & \mathrm{~T} & \mathrm{~T}\end{array}$

3) $\mathrm{Na}_{2} \mathrm{O}-\mathrm{Co}(111)-2 \times 2$

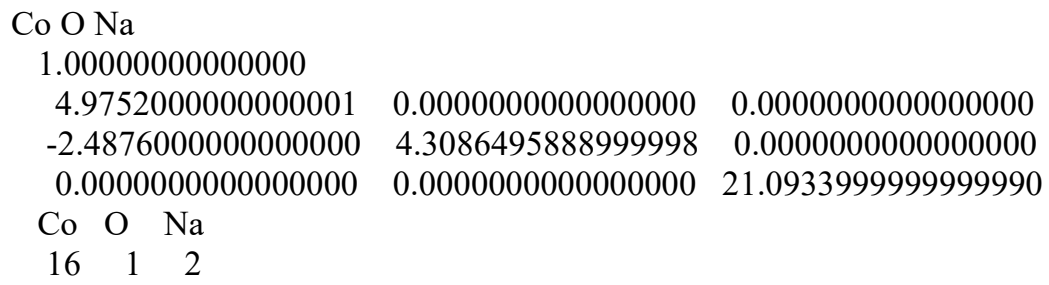

Selective dynamics

Direct

$\begin{array}{lllllllll}0.4445036999999985 & 0.4722397999999970 & 0.0024680000000004 & F & F & F\end{array}$ $\begin{array}{lllllllll}0.9445036999999985 & 0.4722397999999970 & 0.0024680000000004 & F & F & F\end{array}$ $\begin{array}{llllllll}0.4445036999999985 & 0.9722398999999982 & 0.0024680000000004 & F & F & F\end{array}$ $\begin{array}{lllllll}0.9445036999999985 & 0.9722398999999982 & 0.0024680000000004 & F & F & F\end{array}$ $\begin{array}{llllllll}0.11145129999999988 & 0.3057536000000027 & 0.09485509999999666 & F & F & F\end{array}$ $\begin{array}{llllllll}0.6114512999999988 & 0.3057536000000027 & 0.0948550999999966 & F & F & F\end{array}$ $\begin{array}{llllllll}0.11145129999999988 & 0.8057536000000027 & 0.0948550999999966 & \mathrm{~F} & \mathrm{~F} & \mathrm{~F}\end{array}$ $\begin{array}{llllllll}0.6114512999999988 & 0.8057536000000027 & 0.0948550999999966 & F & F & F\end{array}$ $\begin{array}{llllllllll}0.2776521855817350 & 0.1381852048054523 & 0.1930355853846610 & \text { T } & \text { T } & \text { T }\end{array}$ $\begin{array}{lllllll}0.7784782771831753 & 0.1359161925104969 & 0.1935274566575672 & \mathrm{~T} & \mathrm{~T} & \mathrm{~T}\end{array}$ $\begin{array}{llllllll}0.2781755830846162 & 0.6413971381731766 & 0.1922670644672179 & \mathrm{~T} & \mathrm{~T} & \mathrm{~T}\end{array}$ $\begin{array}{lllllll}0.7784940572443121 & 0.6414059959255419 & 0.1930918701225801 & \mathrm{~T} & \mathrm{~T} & \mathrm{~T}\end{array}$ $\begin{array}{llllllll}0.4376959212810057 & 0.4736629485519732 & 0.2852953436066404 & \mathrm{~T} & \mathrm{~T} & \mathrm{~T}\end{array}$ $\begin{array}{llllllll}0.9414012107139454 & 0.4704683161905035 & 0.2824699997488989 & \mathrm{~T} & \mathrm{~T} & \mathrm{~T}\end{array}$ $\begin{array}{llllllll}0.4454287581270113 & 0.9716778432436751 & 0.2883652703952925 & \mathrm{~T} & \mathrm{~T} & \mathrm{~T}\end{array}$ $\begin{array}{llllllll}0.9502930257784340 & 0.9725144480852879 & 0.2931521944187467 & \mathrm{~T} & \mathrm{~T} & \mathrm{~T}\end{array}$ $\begin{array}{llllllllll}0.6039263627627998 & 0.7947550804827977 & 0.3527009919514289 & \mathrm{~T} & \mathrm{~T} & \mathrm{~T}\end{array}$ $\begin{array}{lllllllllll}0.2071085627995353 & 0.6783597355252474 & 0.4178522269062981 & \mathrm{~T} & \mathrm{~T} & \mathrm{~T}\end{array}$ $\begin{array}{llllllllll}0.5498419048112312 & 0.3488324443604114 & 0.4050519521764563 & \mathrm{~T} & \mathrm{~T} & \mathrm{~T}\end{array}$

4) $\mathrm{Na}_{2} \mathrm{O}-\mathrm{Co}(211)-1 \times 2$

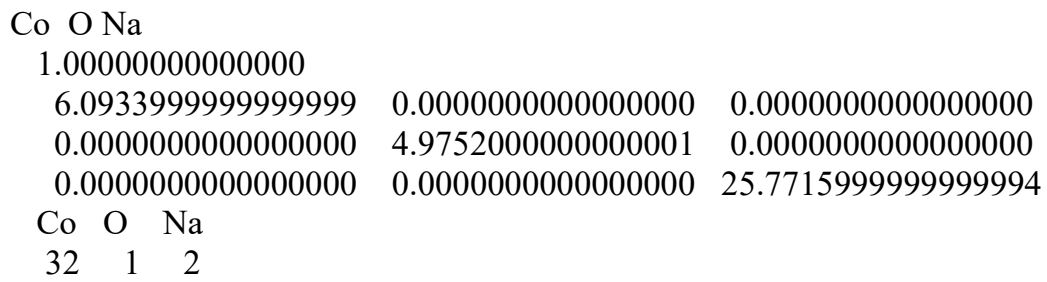

Selective dynamics

Direct

$\begin{array}{lllllll}0.3012132799999989 & 0.0000000000000000 & 0.0046948579056121 & F & F & F\end{array}$ $\begin{array}{lllllllll}0.3012132799999989 & 0.5000000000000000 & 0.0046948579056121 & F & F & F\end{array}$ $\begin{array}{lllllll}0.6286634719999995 & 0.2500000000000000 & 0.0308760418445075 & F & F & F\end{array}$ $\begin{array}{llllllll}0.6286634719999995 & 0.7500000000000000 & 0.0308760418445075 & F & F & F\end{array}$ $\begin{array}{llllllll}0.9633304890000005 & 0.0000000000000000 & 0.0539849291468073 & F & F & F\end{array}$ $\begin{array}{llllllll}0.9633304890000005 & 0.5000000000000000 & 0.0539849291468073 & F & F & F\end{array}$ 
$\begin{array}{llllllll}0.2960606560000016 & 0.2500000000000000 & 0.0837748529388875 & F & F & F\end{array}$ $\begin{array}{lllllll}0.2960606560000016 & 0.7500000000000000 & 0.0837748529388875 & F & F & F\end{array}$ $\begin{array}{lllllllll}0.6289869370000005 & 0.0000000000000000 & 0.1118942944947179 & F & F & F\end{array}$ $\begin{array}{lllllllll}0.6289869370000005 & 0.5000000000000000 & 0.1118942944947179 & F & F & F\end{array}$ $\begin{array}{llllllll}0.9626366230000016 & 0.2500000000000000 & 0.1384130593366422 & F & F & F\end{array}$ $\begin{array}{lllllll}0.9626366230000016 & 0.7500000000000000 & 0.1384130593366422 & F & F & F\end{array}$ $\begin{array}{llllllll}0.2974300059999990 & 0.0000000000000000 & 0.1677073600397421 & F & F & F\end{array}$ $\begin{array}{lllllllll}0.2974300059999990 & 0.5000000000000000 & 0.1677073600397421 & F & F & F\end{array}$ $\begin{array}{llllllll}0.6301967699999977 & 0.2500000000000000 & 0.1957913361995409 & F & F & F\end{array}$ $\begin{array}{llllllllll}0.6301967699999977 & 0.7500000000000000 & 0.1957913361995409 & F & F & F\end{array}$ $\begin{array}{llllllll}0.9619978788376849 & -0.0003490539629597 & 0.2221417784026110 & \text { T } & \text { T } & \text { T }\end{array}$ $\begin{array}{lllllllll}0.9606365063938908 & 0.4996067533403309 & 0.2216319089297358 & \mathrm{~T} & \mathrm{~T} & \mathrm{~T}\end{array}$ $\begin{array}{llllllll}0.2934335824692851 & 0.2502414615288501 & 0.2505753684110027 & \mathrm{~T} & \mathrm{~T} & \mathrm{~T}\end{array}$ $\begin{array}{llllllll}0.2939168337344539 & 0.7486304575455618 & 0.2507821647662609 & \mathrm{~T} & \mathrm{~T} & \mathrm{~T}\end{array}$ $\begin{array}{llllllll}0.6289541009653027 & -0.0005456994246533 & 0.2797308738848672 & \mathrm{~T} & \mathrm{~T} & \mathrm{~T}\end{array}$ $\begin{array}{llllllllll}0.6255320386667752 & 0.4990560074259988 & 0.2795970363039872 & \mathrm{~T} & \mathrm{~T} & \mathrm{~T}\end{array}$ $\begin{array}{lllllllll}0.9613166009727856 & 0.2533160153964640 & 0.3055465320799379 & \mathrm{~T} & \mathrm{~T} & \mathrm{~T}\end{array}$ $\begin{array}{llllllll}0.9626608326994592 & 0.7459595917245979 & 0.3052787713899660 & \mathrm{~T} & \mathrm{~T} & \mathrm{~T}\end{array}$ $\begin{array}{llllllll}0.2948539972108672 & 0.0019008051795465 & 0.3340351808133730 & \mathrm{~T} & \mathrm{~T} & \mathrm{~T}\end{array}$ $\begin{array}{lllllllll}0.2929097363045683 & 0.4975662258682926 & 0.3347014665044555 & \mathrm{~T} & \mathrm{~T} & \mathrm{~T}\end{array}$ $\begin{array}{llllllllll}0.6230062937322909 & 0.2536507104197284 & 0.3629776697897348 & \mathrm{~T} & \mathrm{~T} & \mathrm{~T}\end{array}$ $\begin{array}{lllllllll}0.6253553230172375 & 0.7463329594566810 & 0.3626999500570093 & \mathrm{~T} & \mathrm{~T} & \mathrm{~T}\end{array}$ $\begin{array}{lllllll}0.9576911850290702 & -0.0022316683258602 & 0.3835783501964002 & \mathrm{~T} & \mathrm{~T} & \mathrm{~T}\end{array}$ $\begin{array}{lllllllll}0.9607062413742035 & 0.4996541716991075 & 0.3888888756724684 & \mathrm{~T} & \mathrm{~T} & \mathrm{~T}\end{array}$ $\begin{array}{llllllll}0.2751859196504718 & 0.2415330201427859 & 0.4183535254682993 & \mathrm{~T} & \mathrm{~T} & \mathrm{~T}\end{array}$ $\begin{array}{llllllll}0.2919632663196226 & 0.7541449270527810 & 0.4149315122915813 & \mathrm{~T} & \mathrm{~T} & \mathrm{~T}\end{array}$ $\begin{array}{llllllllll}0.3130056802450303 & 0.9883250648918248 & 0.4724912681101834 & \mathrm{~T} & \mathrm{~T} & \mathrm{~T}\end{array}$ $\begin{array}{llllllllll}0.6831934584206851 & 0.0538965219853949 & 0.4720938927767471 & \mathrm{~T} & \mathrm{~T} & \mathrm{~T}\end{array}$ $\begin{array}{llllllllll}0.1613909137757520 & 0.5974497287993746 & 0.5095076993344319 & \mathrm{~T} & \mathrm{~T} & \mathrm{~T}\end{array}$

5) $\mathrm{PrO}_{2}-\mathrm{Co}(111)-3 \times 3$

Co111_3X3

1.00000000000000

$\begin{array}{llll}7.4587998390197754 & 0.0000000000000000 & 0.0000000000000000\end{array}$

$\begin{array}{llll}-3.7294002959821051 & 6.4595099249780574 & 0.0000000000000000\end{array}$

$\begin{array}{lll}-0.0000010106117090 & -0.0000017504309440 & 23.1201000213622159\end{array}$

Co $\operatorname{Pr} \mathrm{O}$

$\begin{array}{lll}36 & 1 & 2\end{array}$

Selective dynamics

Direct

$\begin{array}{llllllll}0.0000352770987675 & 0.9999648825964869 & 0.0907110695049838 & F & F & F\end{array}$

$\begin{array}{lllllllll}0.3333557427970177 & 0.0000001793936022 & 0.0907175141137841 & F & F & F\end{array}$

$\begin{array}{lllllll}0.6666918534736581 & 0.9999938321653516 & 0.0907267268766958 & F & F & F\end{array}$ $\begin{array}{llllllll}0.0000062578975388 & 0.3333084372988537 & 0.0907267268766958 & F & F & F\end{array}$ $\begin{array}{lllllllll}0.3333357007148621 & 0.3333426504206471 & 0.0907265971194704 & F & F & F\end{array}$ $\begin{array}{llllllll}0.6666945969032270 & 0.3333051862794676 & 0.0907313116319486 & F & F & F\end{array}$ $\begin{array}{lllllllll}0.9999998575876035 & 0.6666444062796870 & 0.0907175141137841 & F & F & F\end{array}$ $\begin{array}{lllllllll}0.3333385397113773 & 0.6666614354387903 & 0.0907302303217463 & F & F & F\end{array}$ $\begin{array}{lllllllll}0.6666573639132167 & 0.6666642220269168 & 0.0907265971194704 & F & F & F\end{array}$ $\begin{array}{llllllll}0.1111175321760314 & 0.2222028177229376 & 0.1750651163386081 & F & F & F\end{array}$ $\begin{array}{lllllll}0.4444257597051688 & 0.2222195372577005 & 0.1750655488626904 & F & F & F\end{array}$ $\begin{array}{llllllll}0.7777781372906531 & 0.2222218594174734 & 0.1750735938105805 & F & F & F\end{array}$ $\begin{array}{llllllll}0.1111123692640206 & 0.5555522552209098 & 0.1750674087162309 & F & F & F\end{array}$ 
$\begin{array}{llllllll}0.4444440697289238 & 0.5555561254847916 & 0.1750719502190776 & F & F & F\end{array}$ $\begin{array}{lllllll}0.7777804782179913 & 0.5555742383122606 & 0.1750655488626904 & F & F & F\end{array}$ $\begin{array}{llllllll}0.1111199658994551 & 0.8888800192425492 & 0.1750494157144900 & F & F & F\end{array}$ $\begin{array}{llllllll}0.4444476042812084 & 0.8888872953412061 & 0.1750674087162309 & F & F & F\end{array}$ $\begin{array}{lllllllll}0.7777970141657633 & 0.8888823414043898 & 0.1750651163386081 & F & F & F\end{array}$ $\begin{array}{llllllll}0.2205507349655056 & 0.1094534330080543 & 0.2654766529655608 & \mathrm{~T} & \mathrm{~T} & \mathrm{~T}\end{array}$ $\begin{array}{llllllll}0.5554005973357555 & 0.1113674967882625 & 0.2649708104635769 & \mathrm{~T} & \mathrm{~T} & \mathrm{~T}\end{array}$ $\begin{array}{llllllll}0.8896503450394350 & 0.1122865792695520 & 0.26588699550440838 & \mathrm{~T} & \mathrm{~T} & \mathrm{~T}\end{array}$ $\begin{array}{lllllllllll}0.2179021402998755 & 0.4457232492530531 & 0.2640837498937665 & \mathrm{~T} & \mathrm{~T} & \mathrm{~T}\end{array}$ $\begin{array}{llllllll}0.5584798439606136 & 0.4445939023372237 & 0.2633480531223145 & \mathrm{~T} & \mathrm{~T} & \mathrm{~T}\end{array}$ $\begin{array}{llllllllll}0.8901559781317090 & 0.4448783373487781 & 0.2669891098999089 & \mathrm{~T} & \mathrm{~T} & \mathrm{~T}\end{array}$ $\begin{array}{llllllll}0.2196254967451199 & 0.7749854356544967 & 0.2666214237726218 & \mathrm{~T} & \mathrm{~T} & \mathrm{~T}\end{array}$ $\begin{array}{lllllllll}0.5537604336581013 & 0.7775207297538278 & 0.2652377348272291 & \mathrm{~T} & \mathrm{~T} & \mathrm{~T}\end{array}$ $\begin{array}{lllllll}0.8897381939651556 & 0.7773281259294238 & 0.2657989644324691 & \mathrm{~T} & \mathrm{~T} & \mathrm{~T}\end{array}$ $\begin{array}{llllllll}0.9925022627991740 & -0.0063480794855958 & 0.3517869473176718 & \mathrm{~T} & \mathrm{~T} & \mathrm{~T}\end{array}$ $\begin{array}{lllllll}0.3373528351197560 & -0.0029213908661792 & 0.3509113587001192 & \mathrm{~T} & \mathrm{~T} & \mathrm{~T}\end{array}$ $\begin{array}{llllllll}0.6649668686147857 & -0.0006575821916713 & 0.3492862213176870 & \mathrm{~T} & \mathrm{~T} & \mathrm{~T}\end{array}$ $\begin{array}{llllllll}-0.0068948876795418 & 0.3296442740893500 & 0.3543386472388838 & \text { T } & \text { T } & \text { T }\end{array}$ $\begin{array}{llllllll}0.3393333738924498 & 0.3408694234210600 & 0.3456303495064795 & \mathrm{~T} & \mathrm{~T} & \mathrm{~T}\end{array}$ $\begin{array}{lllllllll}0.6638482412461191 & 0.3313166729382071 & 0.3503934128369377 & \mathrm{~T} & \mathrm{~T} & \mathrm{~T}\end{array}$ $\begin{array}{llllllll}-0.0062826353240214 & 0.6677193866483342 & 0.3545378466138289 & \mathrm{~T} & \mathrm{~T} & \mathrm{~T}\end{array}$ $\begin{array}{llllllll}0.3378884539168539 & 0.6684976163161643 & 0.3531670235977714 & \mathrm{~T} & \mathrm{~T} & \mathrm{~T}\end{array}$ $\begin{array}{llllllllll}0.6654273185013881 & 0.6649922329133168 & 0.3483132922319320 & \mathrm{~T} & \mathrm{~T} & \mathrm{~T}\end{array}$ $\begin{array}{llllllllll}0.2925201052479974 & 0.2097423465642124 & 0.4515667883134011 & \mathrm{~T} & \mathrm{~T} & \mathrm{~T}\end{array}$ $\begin{array}{llllllll}0.2236252164455444 & 0.4423866230300575 & 0.4102540780261008 & \mathrm{~T} & \mathrm{~T} & \mathrm{~T}\end{array}$ $\begin{array}{llllllll}0.1141684589323647 & -0.1041958024132253 & 0.4127388392188282 & \mathrm{~T} & \mathrm{~T} & \mathrm{~T}\end{array}$

6) $\mathrm{PrO}_{2}-\mathrm{Co}(211)-1 \mathrm{x} 3$

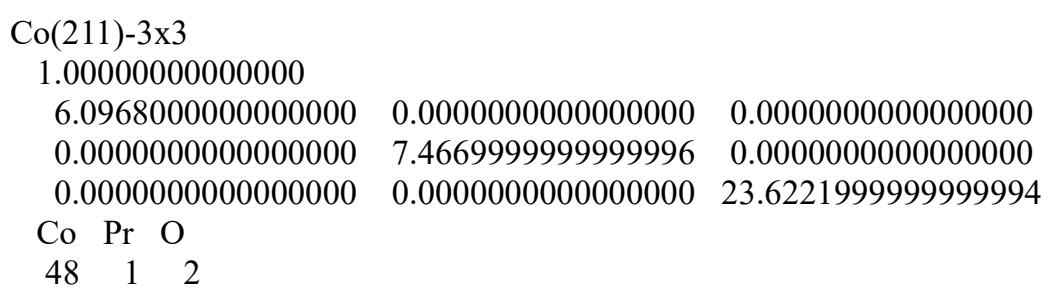

Selective dynamics

Direct

$\begin{array}{lllllllll}0.0039768403096687 & 0.1666663988214836 & 0.9745822997011260 & F & F & F\end{array}$ $\begin{array}{llllllll}0.0039765122687285 & 0.5000000000000000 & 0.9745820457027676 & F & F & F\end{array}$ $\begin{array}{llllllll}0.0039768403096687 & 0.83333336011785164 & 0.9745822997011260 & F & F & F\end{array}$ $\begin{array}{llllllll}0.3315888662905095 & 0.0000000000000000 & 0.0034278771663949 & F & F & F\end{array}$ $\begin{array}{lllllll}0.3315855858811148 & 0.3333350743270387 & 0.0034291471581795 & F & F & F\end{array}$ $\begin{array}{lllllllll}0.3315855858811148 & 0.6666647917503710 & 0.0034291471581795 & \mathrm{~F} & \mathrm{~F} & \mathrm{~F}\end{array}$ $\begin{array}{llllllll}0.6664853693741009 & 0.1666655952859273 & 0.0285661792720404 & F & F & F\end{array}$ $\begin{array}{llllllll}0.6664884857630256 & 0.5000000000000000 & 0.0285654596100287 & F & F & F\end{array}$ $\begin{array}{llllllll}0.6664853693741009 & 0.83333444047140727 & 0.0285661792720404 & F & F & F\end{array}$ $\begin{array}{lllllllll}0.9983825941477491 & 0.0000000000000000 & 0.0609119811025209 & F & F & F\end{array}$ $\begin{array}{llllllll}0.9983791497178842 & 0.33333453386366702 & 0.0609079171288016 & F & F & F\end{array}$ $\begin{array}{llllllll}0.9983791497178842 & 0.6666654613633298 & 0.0609079171288016 & F & F & F\end{array}$ $\begin{array}{llllllll}0.3314264860254568 & 0.1666650595955517 & 0.0918800111759310 & F & F & F\end{array}$ $\begin{array}{llllllll}0.3314281262301506 & 0.5000000000000000 & 0.0918805615057039 & F & F & F\end{array}$ $\begin{array}{lllllll}0.3314264860254568 & 0.8333349404044483 & 0.0918800111759310 & F & F & F\end{array}$ $\begin{array}{llllllll}0.6654751673008761 & 0.0000000000000000 & 0.1205095207050988 & F & F & F\end{array}$ 
$\begin{array}{llllllll}0.6654631938065876 & 0.3333330654881479 & 0.1205040597404121 & F & F & F\end{array}$ $\begin{array}{llllllll}0.6654631938065876 & 0.6666669345118521 & 0.1205040597404121 & F & F & F\end{array}$ $\begin{array}{llllllll}0.0009121178323070 & 0.1666669345118521 & 0.1526111031148645 & F & F & F\end{array}$ $\begin{array}{llllllll}0.0009114617504267 & 0.5000000000000000 & 0.1526112301140472 & F & F & F\end{array}$ $\begin{array}{lllllll}0.0009121178323070 & 0.8333330654881479 & 0.1526111031148645 & F & F & F\end{array}$ $\begin{array}{lllllllll}0.3340214538774404 & 0.0000000000000000 & 0.1833969740329024 & F & F & F\end{array}$ $\begin{array}{llllllllll}0.3340229300616713 & 0.3333327976429601 & 0.1833968470337197 & F & F & F\end{array}$ $\begin{array}{lllllllll}0.3340229300616713 & 0.6666672023570399 & 0.1833968470337197 & F & F & F\end{array}$ $\begin{array}{llllllll}0.6647434005567515 & 0.1665774013671639 & 0.2117360831755252 & \mathrm{~T} & \mathrm{~T} & \mathrm{~T}\end{array}$ $\begin{array}{llllllll}0.6661519577181387 & 0.4996734608718044 & 0.2119139648101470 & \mathrm{~T} & \mathrm{~T} & \mathrm{~T}\end{array}$ $\begin{array}{llllllll}0.6659141096602573 & 0.8342002262019684 & 0.2118690743323975 & \mathrm{~T} & \mathrm{~T} & \mathrm{~T}\end{array}$ $\begin{array}{llllllll}0.9964393114311343 & 0.0009227820541037 & 0.2431542600300359 & \mathrm{~T} & \mathrm{~T} & \mathrm{~T}\end{array}$ $\begin{array}{lllllll}-0.0011432956328016 & 0.3333778118860782 & 0.2429869856777780 & \mathrm{~T} & \mathrm{~T} & \mathrm{~T}\end{array}$ $\begin{array}{lllllll}-0.0002089003064927 & 0.6661987203426186 & 0.2427287716086656 & \mathrm{~T} & \mathrm{~T} & \mathrm{~T}\end{array}$ $\begin{array}{lllllll}0.3314599722444967 & 0.1671835516208988 & 0.2757971695707473 & \mathrm{~T} & \mathrm{~T} & \mathrm{~T}\end{array}$ $\begin{array}{llllllll}0.3345632533814381 & 0.4985795541405580 & 0.2755451716167761 & \mathrm{~T} & \mathrm{~T} & \mathrm{~T}\end{array}$ $\begin{array}{llllllll}0.3318229527220464 & 0.8350489727305822 & 0.2736918115721638 & \mathrm{~T} & \mathrm{~T} & \mathrm{~T}\end{array}$ $\begin{array}{lllllll}0.6649016366256801 & 0.0018221812058383 & 0.3035486815530158 & \mathrm{~T} & \mathrm{~T} & \mathrm{~T}\end{array}$ $\begin{array}{llllllll}0.6682134036001685 & 0.3294688816297060 & 0.3021783541257580 & \mathrm{~T} & \mathrm{~T} & \mathrm{~T}\end{array}$ $\begin{array}{llllllll}0.6659778889987896 & 0.6676940121105575 & 0.3019670862987719 & \mathrm{~T} & \mathrm{~T} & \mathrm{~T}\end{array}$ $\begin{array}{llllllll}0.9982792811797545 & 0.1684834865515520 & 0.3353824638116968 & \mathrm{~T} & \mathrm{~T} & \mathrm{~T}\end{array}$ $\begin{array}{llllllll}0.0029077364228150 & 0.4989424816448920 & 0.3347768770371820 & \mathrm{~T} & \mathrm{~T} & \mathrm{~T}\end{array}$ $\begin{array}{llllllll}0.9973720717684887 & 0.8325619969512291 & 0.3343715689230554 & \mathrm{~T} & \mathrm{~T} & \mathrm{~T}\end{array}$ $\begin{array}{llllllll}0.3283272693411702 & 0.9965530945411605 & 0.3663266051835712 & \mathrm{~T} & \mathrm{~T} & \mathrm{~T}\end{array}$ $\begin{array}{llllllll}0.3256942897623311 & 0.3313773457506297 & 0.3697735229751465 & \mathrm{~T} & \mathrm{~T} & \mathrm{~T}\end{array}$ $\begin{array}{llllllll}0.3274083206636151 & 0.6745745372857700 & 0.3642271721797583 & \mathrm{~T} & \mathrm{~T} & \mathrm{~T}\end{array}$ $\begin{array}{lllllll}0.6711602876081163 & 0.1608480675955824 & 0.3966851276477509 & \mathrm{~T} & \mathrm{~T} & \mathrm{~T}\end{array}$ $\begin{array}{llllllll}0.6699000001952580 & 0.5008209129673641 & 0.3872377107990902 & \mathrm{~T} & \mathrm{~T} & \mathrm{~T}\end{array}$ $\begin{array}{llllllll}0.6639892542033518 & 0.8347984798637287 & 0.3912170579799690 & \mathrm{~T} & \mathrm{~T} & \mathrm{~T}\end{array}$ $\begin{array}{llllllll}0.9950575859126305 & 0.9952136632865903 & 0.4202637815395033 & \mathrm{~T} & \mathrm{~T} & \mathrm{~T}\end{array}$ $\begin{array}{llllllll}0.9970782071186626 & 0.3386946583382129 & 0.4265180209270397 & \mathrm{~T} & \mathrm{~T} & \mathrm{~T}\end{array}$ $\begin{array}{llllllll}0.9756689453573376 & 0.6708183985952250 & 0.4239015393492523 & \mathrm{~T} & \mathrm{~T} & \mathrm{~T}\end{array}$ $\begin{array}{lllllll}0.4024443334021542 & 0.5932255357087505 & 0.4823653893281696 & \mathrm{~T} & \mathrm{~T} & \mathrm{~T}\end{array}$ $\begin{array}{llllllll}0.5172612014174336 & 0.3406377852776554 & 0.4413523363336903 & \mathrm{~T} & \mathrm{~T} & \mathrm{~T}\end{array}$ $\begin{array}{lllllll}0.0514696918509387 & 0.5117638694791101 & 0.4847024061000213 & \mathrm{~T} & \mathrm{~T} & \mathrm{~T}\end{array}$

7) $\mathrm{Na}_{2} \mathrm{O}-\mathrm{Co}(111)-3 \times 3$

$\mathrm{CoO} \mathrm{Oa}$

1.00000000000000

$\begin{array}{llll}7.4587998390197754 & 0.0000000000000000 & 0.0000000000000000\end{array}$

$\begin{array}{llll}-3.7294002959821051 & 6.4595099249780574 & 0.0000000000000000\end{array}$

$\begin{array}{lll}-0.0000010106117090 & -0.0000017504309440 & 23.1201000213622159\end{array}$

Co $\mathrm{O} \quad \mathrm{Na}$

$\begin{array}{lll}36 & 1 & 2\end{array}$

Selective dynamics

Direct

$\begin{array}{llllllll}0.0000353225999987 & 0.9999648941999979 & 0.0907110695000028 & \mathrm{~F} & \mathrm{~F} & \mathrm{~F}\end{array}$ $\begin{array}{llllllll}0.3333557428000020 & 0.0000001793999971 & 0.0907175140999996 & F & F & F\end{array}$ $\begin{array}{llllllll}0.6666917649000013 & 0.9999938437999987 & 0.0907267269000016 & F & F & F\end{array}$ $\begin{array}{llllllll}0.0000062578999973 & 0.3333084372999977 & 0.0907267269000016 & F & F & F\end{array}$ $\begin{array}{lllllllll}0.3333357006999975 & 0.3333426503999988 & 0.0907265971000015 & \mathrm{~F} & \mathrm{~F} & \mathrm{~F}\end{array}$ $\begin{array}{lllllllll}0.6666945969000011 & 0.3333051863000023 & 0.0907313116000026 & F & F & F\end{array}$ $\begin{array}{lllllllll}0.9999998791999971 & 0.6666444063000014 & 0.0907175140999996 & F & F & F\end{array}$ 
$\begin{array}{llllllll}0.3333385397000015 & 0.6666614354000018 & 0.0907302302999966 & F & F & F\end{array}$ $\begin{array}{llllllll}0.6666573639000006 & 0.6666642220000014 & 0.0907265971000015 & F & F & F\end{array}$ $\begin{array}{lllllll}0.1111175321999980 & 0.2222028177000013 & 0.1750651162999972 & F & F & F\end{array}$ $\begin{array}{llllllll}0.4444257597000032 & 0.2222195372999991 & 0.1750655489000010 & F & F & F\end{array}$ $\begin{array}{lllllllll}0.7777781372999968 & 0.2222218594000012 & 0.1750735938000005 & F & F & F\end{array}$ $\begin{array}{llllllll}0.1111123693000025 & 0.5555522551999985 & 0.1750674087000021 & F & F & F\end{array}$ $\begin{array}{llllllll}0.4444440696999976 & 0.5555561254999972 & 0.17507195019999995 & F & F & F\end{array}$ $\begin{array}{lllllll}0.7777804782000004 & 0.5555742382999966 & 0.1750655489000010 & F & F & F\end{array}$ $\begin{array}{llllllll}0.1111199659000022 & 0.8888800192000019 & 0.1750494157000020 & F & F & F\end{array}$ $\begin{array}{llllllll}0.4444476043000023 & 0.8888872953000018 & 0.1750674087000021 & F & F & F\end{array}$ $\begin{array}{lllllllll}0.7777970141999972 & 0.8888823413999987 & 0.1750651162999972 & F & F & F\end{array}$ $\begin{array}{llllllll}0.2221375239702549 & 0.1108236384194744 & 0.2657001400775192 & \mathrm{~T} & \mathrm{~T} & \mathrm{~T}\end{array}$ $\begin{array}{llllllll}0.5538450150951526 & 0.1095131094696231 & 0.2648837721616484 & \mathrm{~T} & \mathrm{~T} & \mathrm{~T}\end{array}$ $\begin{array}{lllllll}0.8889445048430319 & 0.1090274766311578 & 0.2645677596331018 & \mathrm{~T} & \mathrm{~T} & \mathrm{~T}\end{array}$ $\begin{array}{llllllll}0.2242068901455473 & 0.4451793931308817 & 0.2663897570801773 & \mathrm{~T} & \mathrm{~T} & \mathrm{~T}\end{array}$ $\begin{array}{llllllll}0.5552506221723262 & 0.4443108394725988 & 0.2656473229574078 & \mathrm{~T} & \mathrm{~T} & \mathrm{~T}\end{array}$ $\begin{array}{llllllll}0.8893721638800284 & 0.4460756912415831 & 0.2641928419378674 & \mathrm{~T} & \mathrm{~T} & \mathrm{~T}\end{array}$ $\begin{array}{llllllll}0.2228258765026604 & 0.7771693752484282 & 0.2659593443576885 & \mathrm{~T} & \mathrm{~T} & \mathrm{~T}\end{array}$ $\begin{array}{llllllll}0.5538708322168195 & 0.7772885041014707 & 0.2660576322194308 & \mathrm{~T} & \mathrm{~T} & \mathrm{~T}\end{array}$ $\begin{array}{lllllllllll}0.8889343303881384 & 0.7777209264526380 & 0.2654570282682344 & \mathrm{~T} & \mathrm{~T} & \mathrm{~T}\end{array}$ $\begin{array}{llllllll}-0.0003289555853119 & 0.0002457014848329 & 0.3506051186187231 & \mathrm{~T} & \mathrm{~T} & \mathrm{~T}\end{array}$ $\begin{array}{lllllllll}0.3338314578165443 & -0.0008273913146767 & 0.3501954245568780 & \mathrm{~T} & \mathrm{~T} & \mathrm{~T}\end{array}$ $\begin{array}{llllllllll}0.6701152097306753 & 0.0057977508861954 & 0.3499077571317304 & \mathrm{~T} & \mathrm{~T} & \mathrm{~T}\end{array}$ $\begin{array}{llllllll}0.9928932049338628 & 0.3304678726635799 & 0.3476629385132990 & \text { T } & \text { T } & \text { T }\end{array}$ $\begin{array}{llllllllll}0.3355696877805055 & 0.3303691952865750 & 0.3528461434693643 & \mathrm{~T} & \mathrm{~T} & \mathrm{~T}\end{array}$ $\begin{array}{llllllll}0.6703716961885422 & 0.3305174698880314 & 0.3488655271307697 & \mathrm{~T} & \mathrm{~T} & \mathrm{~T}\end{array}$ $\begin{array}{llllllll}0.0001064177965198 & 0.6646029288281463 & 0.3503147932921035 & \mathrm{~T} & \mathrm{~T} & \mathrm{~T}\end{array}$ $\begin{array}{llllllll}0.3329702395991189 & 0.6664923141241721 & 0.3549101549016222 & \mathrm{~T} & \mathrm{~T} & \mathrm{~T}\end{array}$ $\begin{array}{lllllllll}0.6665515438554770 & 0.6696537078962550 & 0.3508712602443770 & \mathrm{~T} & \mathrm{~T} & \mathrm{~T}\end{array}$ $\begin{array}{lllllllll}0.4496804987777677 & 0.5532233989202600 & 0.4107895599536002 & \mathrm{~T} & \mathrm{~T} & \mathrm{~T}\end{array}$ $\begin{array}{llllllll}0.1389244463031845 & 0.3606218161921505 & 0.4581212788291053 & \mathrm{~T} & \mathrm{~T} & \mathrm{~T}\end{array}$ $\begin{array}{lllllllllll}0.7415198015536109 & 0.5852251281331488 & 0.4586396278861144 & \mathrm{~T} & \mathrm{~T} & \mathrm{~T}\end{array}$

8) $\mathrm{Na}_{2} \mathrm{O}-\mathrm{Co}(211)-1 \times 3$

Co(211)-3x3

1.00000000000000

$\begin{array}{rrr}6.0968000000000000 & 0.0000000000000000 & 0.0000000000000000\end{array}$

$\begin{array}{llll}0.0000000000000000 & 7.46699999999999996 & 0.0000000000000000\end{array}$

$\begin{array}{llll}0.0000000000000000 & 0.0000000000000000 & 23.6221999999999994\end{array}$

Co $\mathrm{O} \quad \mathrm{Na}$

$\begin{array}{lll}48 & 1 & 2\end{array}$

Selective dynamics

Direct

$\begin{array}{lllllllll}0.0039768403096687 & 0.1666663988214765 & 0.9745822997011331 & F & F & F\end{array}$ $\begin{array}{llllllll}0.0039765122687285 & 0.5000000000000000 & 0.9745822997011331 & F & F & F\end{array}$ $\begin{array}{llllllll}0.0039768403096687 & 0.83333360011785235 & 0.9745822997011331 & F & F & F\end{array}$ $\begin{array}{llllllll}0.3315888662905095 & 0.0000000000000000 & 0.0034278771663878 & F & F & F\end{array}$ $\begin{array}{lllllll}0.3315855858811219 & 0.3333350743270387 & 0.0034291471581795 & F & F & F\end{array}$ $\begin{array}{llllllll}0.3315855858811219 & 0.6666647917503710 & 0.0034291471581795 & F & F & F\end{array}$ $\begin{array}{lllllll}0.6664853693741009 & 0.1666655952859273 & 0.0285661792720404 & F & F & F\end{array}$ $\begin{array}{lllllllll}0.6664884857630327 & 0.5000000000000000 & 0.0285654596100287 & F & F & F\end{array}$ $\begin{array}{llllllll}0.6664853693741009 & 0.8333344047140727 & 0.0285661792720404 & \mathrm{~F} & \mathrm{~F} & \mathrm{~F}\end{array}$ $\begin{array}{llllllll}0.9983825941477491 & 0.0000000000000000 & 0.0609119811025209 & F & F & F\end{array}$ 
$\begin{array}{llllllll}0.9983791497178913 & 0.3333345386366702 & 0.0609079171288016 & F & F & F\end{array}$ $\begin{array}{lllllllll}0.9983791497178913 & 0.6666654613633298 & 0.0609079171288016 & F & F & F\end{array}$ $\begin{array}{llllllll}0.3314264860254568 & 0.1666650595955517 & 0.0918800111759310 & F & F & F\end{array}$ $\begin{array}{llllllll}0.3314281262301506 & 0.5000000000000000 & 0.0918805615056968 & F & F & F\end{array}$ $\begin{array}{llllllll}0.3314264860254568 & 0.8333349404044483 & 0.0918800111759310 & F & F & F\end{array}$ $\begin{array}{lllllllll}0.6654751673008832 & 0.0000000000000000 & 0.1205095207050988 & F & F & F\end{array}$ $\begin{array}{llllllll}0.6654631938065876 & 0.3333330654881479 & 0.1205040597404121 & F & F & F\end{array}$ $\begin{array}{llllllll}0.6654631938065876 & 0.6666669345118521 & 0.1205040597404121 & F & F & F\end{array}$ $\begin{array}{llllllll}0.0009121178323070 & 0.1666669345118521 & 0.1526111031148716 & F & F & F\end{array}$ $\begin{array}{lllllllll}0.0009114617504267 & 0.5000000000000000 & 0.1526112301140472 & F & F & F\end{array}$ $\begin{array}{llllllll}0.0009121178323070 & 0.8333330654881479 & 0.1526111031148716 & F & F & F\end{array}$ $\begin{array}{lllllllll}0.3340214538774404 & 0.0000000000000000 & 0.1833969740329024 & F & F & F\end{array}$ $\begin{array}{lllllllll}0.3340229300616713 & 0.3333327976429601 & 0.1833968470337197 & F & F & F\end{array}$ $\begin{array}{lllllllll}0.3340229300616713 & 0.6666672023570399 & 0.1833968470337197 & F & F & F\end{array}$ $\begin{array}{lllllll}0.6655821287910602 & 0.1668431383189340 & 0.2117088250351775 & \mathrm{~T} & \mathrm{~T} & \mathrm{~T}\end{array}$ $\begin{array}{llllllll}0.6668572790929191 & 0.5001748255385742 & 0.2117210293961458 & \mathrm{~T} & \mathrm{~T} & \mathrm{~T}\end{array}$ $\begin{array}{llllllll}0.6656478898741726 & 0.8334977678343171 & 0.2117280225223900 & \mathrm{~T} & \mathrm{~T} & \mathrm{~T}\end{array}$ $\begin{array}{lllllllll}0.9976654652466771 & 0.0002760802133924 & 0.2434013885829756 & \mathrm{~T} & \mathrm{~T} & \mathrm{~T}\end{array}$ $\begin{array}{lllllll}0.9990461964891442 & 0.3333576400088915 & 0.2431517729846123 & \mathrm{~T} & \mathrm{~T} & \mathrm{~T}\end{array}$ $\begin{array}{llllllllll}0.9991068720503014 & 0.6669258733323830 & 0.2431345948961191 & \mathrm{~T} & \mathrm{~T} & \mathrm{~T}\end{array}$ $\begin{array}{lllllllll}0.3322741382127590 & 0.1657073138076697 & 0.2748862694046491 & \mathrm{~T} & \mathrm{~T} & \mathrm{~T}\end{array}$ $\begin{array}{lllllll}0.3332729227843304 & 0.5002925476014702 & 0.2741535734974035 & \mathrm{~T} & \mathrm{~T} & \mathrm{~T}\end{array}$ $\begin{array}{llllllll}0.3324364872068558 & 0.8350908039651603 & 0.2749325767103811 & \mathrm{~T} & \mathrm{~T} & \mathrm{~T}\end{array}$ $\begin{array}{llllllll}0.6660352724136506 & 0.0004368406273403 & 0.3031945854837930 & \mathrm{~T} & \mathrm{~T} & \mathrm{~T}\end{array}$ $\begin{array}{llllllll}0.6677715423790579 & 0.3332722304922451 & 0.3025606624715578 & \mathrm{~T} & \mathrm{~T} & \mathrm{~T}\end{array}$ $\begin{array}{lllllll}0.6677199126723382 & 0.6674864880295392 & 0.3025741510549418 & \mathrm{~T} & \mathrm{~T} & \mathrm{~T}\end{array}$ $\begin{array}{llllllll}0.9995878652569509 & 0.1670625395948408 & 0.3351874023648010 & \mathrm{~T} & \mathrm{~T} & \mathrm{~T}\end{array}$ $\begin{array}{llllllll}-0.0007561895679957 & 0.5005315361135679 & 0.3354785858588274 & \mathrm{~T} & \mathrm{~T} & \mathrm{~T}\end{array}$ $\begin{array}{llllllll}0.9997046719551758 & 0.8340647390133386 & 0.3351816261628597 & \mathrm{~T} & \mathrm{~T} & \mathrm{~T}\end{array}$ $\begin{array}{lllllll}0.3290263867039529 & 0.0006104057561895 & 0.3693972950926733 & \mathrm{~T} & \mathrm{~T} & \mathrm{~T}\end{array}$ $\begin{array}{llllllll}0.3311108846346809 & 0.3326208677232209 & 0.3633912610284071 & \mathrm{~T} & \mathrm{~T} & \mathrm{~T}\end{array}$ $\begin{array}{llllllll}0.3312471216413790 & 0.6682728643536109 & 0.3634031042584279 & \mathrm{~T} & \mathrm{~T} & \mathrm{~T}\end{array}$ $\begin{array}{lllllll}0.6669684752085723 & 0.1665625385715724 & 0.3912602739867361 & \mathrm{~T} & \mathrm{~T} & \mathrm{~T}\end{array}$ $\begin{array}{lllllll}0.6621268316075702 & 0.5005881335914051 & 0.3900951564383917 & \mathrm{~T} & \mathrm{~T} & \mathrm{~T}\end{array}$ $\begin{array}{llllllllll}0.6671220837383890 & 0.8344367243648314 & 0.3911912807950934 & \mathrm{~T} & \mathrm{~T} & \mathrm{~T}\end{array}$ $\begin{array}{llllllll}0.9879154868941545 & 0.0005794670418491 & 0.4213351610769437 & \mathrm{~T} & \mathrm{~T} & \mathrm{~T}\end{array}$ $\begin{array}{lllllll}0.9866982507998551 & 0.3369719419383915 & 0.4255181656644864 & \mathrm{~T} & \mathrm{~T} & \mathrm{~T}\end{array}$ $\begin{array}{lllllll}0.9858227194948890 & 0.6641003444936842 & 0.4253789963442483 & \text { T } & \text { T } & \text { T }\end{array}$ $\begin{array}{lllllll}0.1059669506924052 & 0.5012360443191954 & 0.4771498312023839 & \mathrm{~T} & \mathrm{~T} & \mathrm{~T}\end{array}$ $\begin{array}{llllllll}0.3787526831448976 & 0.7323050011145081 & 0.4844344581755369 & \mathrm{~T} & \mathrm{~T} & \mathrm{~T}\end{array}$ $\begin{array}{lllllll}0.3835036096373457 & 0.2778411376652029 & 0.4846225228807932 & \mathrm{~T} & \mathrm{~T} & \mathrm{~T}\end{array}$

9) $\mathrm{H}-\mathrm{Co}(111)-3 \times 3$

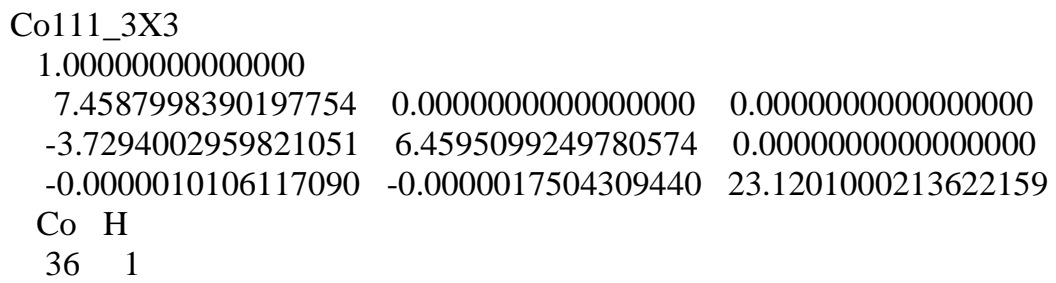

Selective dynamics

Direct

$\begin{array}{llllllll}0.0000353225881327 & 0.9999648942106703 & 0.0907110695049838 & F & F & F\end{array}$ $\begin{array}{llllllll}0.3333557427970177 & 0.0000001793936022 & 0.0907175141137841 & F & F & F\end{array}$ $\begin{array}{llllllll}0.6666917648931943 & 0.9999938437795350 & 0.0907267268766958 & F & F & F\end{array}$ 
$\begin{array}{llllllll}0.0000062578975388 & 0.3333084372988537 & 0.0907267268766958 & F & F & F\end{array}$ $\begin{array}{llllllll}0.3333357007148621 & 0.3333426504206471 & 0.0907265971194704 & F & F & F\end{array}$ $\begin{array}{lllllllll}0.6666945969032270 & 0.3333051862794676 & 0.0907313116319486 & F & F & F\end{array}$ $\begin{array}{llllllll}0.9999998791701969 & 0.6666444062796870 & 0.0907175141137841 & F & F & F\end{array}$ $\begin{array}{lllllllll}0.3333385397113773 & 0.6666614354387903 & 0.0907302303217463 & F & F & F\end{array}$ $\begin{array}{lllllllll}0.6666573639132167 & 0.6666642220269168 & 0.0907265971194704 & F & F & F\end{array}$ $\begin{array}{llllllll}0.1111175321760314 & 0.2222028177229376 & 0.1750651163386081 & F & F & F\end{array}$ $\begin{array}{lllllllll}0.4444257597051688 & 0.2222195372577005 & 0.1750655488626904 & F & F & F\end{array}$ $\begin{array}{llllllll}0.7777781372906531 & 0.2222218594174734 & 0.1750735938105805 & F & F & F\end{array}$ $\begin{array}{lllllllll}0.1111123692640206 & 0.5555522552209098 & 0.1750674087162309 & F & F & F\end{array}$ $\begin{array}{lllllllll}0.4444440697289238 & 0.5555561254847916 & 0.1750719502190776 & F & F & F\end{array}$ $\begin{array}{lllllllll}0.7777804782179913 & 0.5555742383122606 & 0.1750655488626904 & F & F & F\end{array}$ $\begin{array}{lllllllll}0.1111199658994551 & 0.8888800192425492 & 0.1750494157144900 & F & F & F\end{array}$ $\begin{array}{lllllllll}0.4444476042812084 & 0.8888872953412061 & 0.1750674087162309 & F & F & F\end{array}$ $\begin{array}{llllllll}0.7777970141657633 & 0.8888823414043898 & 0.1750651163386081 & F & F & F\end{array}$ $\begin{array}{lllllll}0.2224291914823709 & 0.1114860978844242 & 0.2654663925162335 & \mathrm{~T} & \mathrm{~T} & \mathrm{~T}\end{array}$ $\begin{array}{llllllll}0.5558656833659361 & 0.1112327459103788 & 0.2653033147277880 & \mathrm{~T} & \mathrm{~T} & \mathrm{~T}\end{array}$ $\begin{array}{llllllll}0.8887504041543466 & 0.1112492744758355 & 0.2652816691922229 & \mathrm{~T} & \mathrm{~T} & \mathrm{~T}\end{array}$ $\begin{array}{llllllll}0.2221893963104555 & 0.4443877503904330 & 0.2655908375963801 & \mathrm{~T} & \mathrm{~T} & \mathrm{~T}\end{array}$ $\begin{array}{lllllllll}0.5556521774696990 & 0.4443477858388194 & 0.2655970907706153 & \mathrm{~T} & \mathrm{~T} & \mathrm{~T}\end{array}$ $\begin{array}{llllllll}0.8887672650646076 & 0.4441342206638560 & 0.2653033147277880 & \mathrm{~T} & \mathrm{~T} & \mathrm{~T}\end{array}$ $\begin{array}{llllllll}0.2223941094648475 & 0.7776057166172979 & 0.2654428983024547 & \mathrm{~T} & \mathrm{~T} & \mathrm{~T}\end{array}$ $\begin{array}{llllllll}0.5556123405712013 & 0.7778103591848184 & 0.2655908375963801 & \mathrm{~T} & \mathrm{~T} & \mathrm{~T}\end{array}$ $\begin{array}{lllllllll}0.8885137127344428 & 0.7775707296764752 & 0.2654663925162335 & \mathrm{~T} & \mathrm{~T} & \mathrm{~T}\end{array}$ $\begin{array}{llllllll}-0.0000215697849555 & 0.0000214189168304 & 0.3494035012962458 & \mathrm{~T} & \mathrm{~T} & \mathrm{~T}\end{array}$ $\begin{array}{lllllll}0.3333626060572703 & 0.0000335932143061 & 0.3494121225901914 & \mathrm{~T} & \mathrm{~T} & \mathrm{~T}\end{array}$ $\begin{array}{lllllll}0.6673371618904517 & 0.0012292132406806 & 0.3494884600222356 & \mathrm{~T} & \mathrm{~T} & \mathrm{~T}\end{array}$ $\begin{array}{lllllll}0.9987706651408281 & 0.3326627396522843 & 0.3494884600222356 & \mathrm{~T} & \mathrm{~T} & \mathrm{~T}\end{array}$ $\begin{array}{lllllll}0.3329571874349466 & 0.3325087390401797 & 0.3511022364418594 & \mathrm{~T} & \mathrm{~T} & \mathrm{~T}\end{array}$ $\begin{array}{llllllll}0.6673557599760700 & 0.3326440652483155 & 0.3495047093800124 & \mathrm{~T} & \mathrm{~T} & \mathrm{~T}\end{array}$ $\begin{array}{lllllll}-0.0000334474763423 & 0.6666375947809595 & 0.3494121225901914 & \mathrm{~T} & \mathrm{~T} & \mathrm{~T}\end{array}$ $\begin{array}{lllllll}0.3328327348728022 & 0.6671674380287202 & 0.3510722523910971 & \mathrm{~T} & \mathrm{~T} & \mathrm{~T}\end{array}$ $\begin{array}{lllllll}0.6674912915028620 & 0.6670429447278637 & 0.3511022364418594 & \mathrm{~T} & \mathrm{~T} & \mathrm{~T}\end{array}$ $\begin{array}{lllllll}0.4442402352599193 & 0.5557600786825238 & 0.3933913343539523 & \mathrm{~T} & \mathrm{~T} & \mathrm{~T}\end{array}$

10) $\mathrm{H}-\mathrm{PrO}_{2}-\mathrm{Co}(111)-3 \times 3$

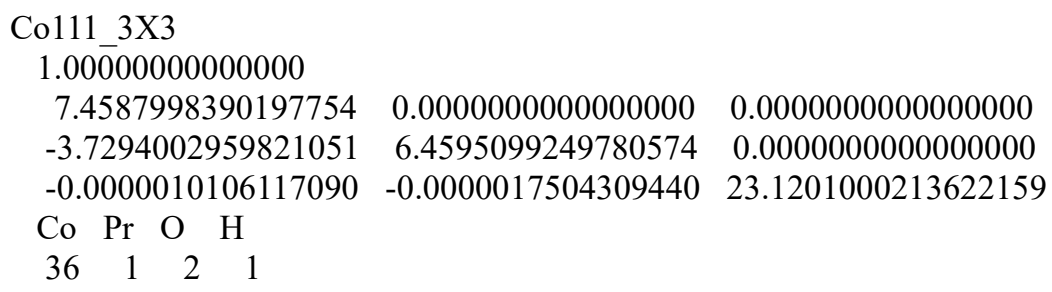

Selective dynamics

Direct

$\begin{array}{llllllll}0.0000353225881327 & 0.9999648942106703 & 0.0907110695049838 & F & F & F\end{array}$ $\begin{array}{llllllll}0.3333557427970177 & 0.0000001793936022 & 0.0907175141137841 & F & F & F\end{array}$ $\begin{array}{lllllllll}0.6666917648931943 & 0.9999938437795350 & 0.0907267268766958 & F & F & F\end{array}$ $\begin{array}{lllllllll}0.0000062578975388 & 0.3333084372988537 & 0.0907267268766958 & F & F & F\end{array}$ $\begin{array}{llllllll}0.3333357007148621 & 0.3333426504206471 & 0.0907265971194704 & F & F & F\end{array}$ $\begin{array}{llllllll}0.6666945969032270 & 0.3333051862794676 & 0.0907313116319486 & F & F & F\end{array}$ $\begin{array}{lllllllll}0.9999998791701969 & 0.6666444062796870 & 0.0907175141137841 & F & F & F\end{array}$ $\begin{array}{llllllll}0.3333385397113773 & 0.6666614354387903 & 0.0907302303217463 & F & F & F\end{array}$ $\begin{array}{lllllllll}0.6666573639132167 & 0.6666642220269168 & 0.0907265971194704 & F & F & F\end{array}$ 
$\begin{array}{lllllll}0.1111175321760314 & 0.2222028177229376 & 0.1750651163386081 & F & F & F\end{array}$ $\begin{array}{llllllll}0.4444257597051688 & 0.2222195372577005 & 0.1750655488626904 & \mathrm{~F} & \mathrm{~F} & \mathrm{~F}\end{array}$ $\begin{array}{lllllllll}0.7777781372906531 & 0.2222218594174734 & 0.1750735938105805 & F & F & F\end{array}$ $\begin{array}{llllllll}0.1111123692640206 & 0.5555522552209098 & 0.1750674087162309 & F & F & F\end{array}$ $\begin{array}{llllllll}0.4444440697289238 & 0.5555561254847916 & 0.1750719502190776 & F & F & F\end{array}$ $\begin{array}{llllllllll}0.7777804782179913 & 0.5555742383122606 & 0.1750655488626904 & \mathrm{~F} & \mathrm{~F} & \mathrm{~F}\end{array}$ $\begin{array}{llllllll}0.1111199658994551 & 0.8888800192425492 & 0.1750494157144900 & F & F & F\end{array}$ $\begin{array}{llllllll}0.4444476042812084 & 0.8888872953412061 & 0.1750674087162309 & F & F & F\end{array}$ $\begin{array}{llllllll}0.7777970141657633 & 0.8888823414043898 & 0.1750651163386081 & F & F & F\end{array}$ $\begin{array}{lllllllll}0.2191479633409761 & 0.1077986947864803 & 0.2644190925534561 & \mathrm{~T} & \mathrm{~T} & \mathrm{~T}\end{array}$ $\begin{array}{llllllll}0.5547020951815628 & 0.1110556478749043 & 0.2650369120607196 & \mathrm{~T} & \mathrm{~T} & \mathrm{~T}\end{array}$ $\begin{array}{lllllllllllll}0.8888881379693437 & 0.1121111827377106 & 0.2657295298291154 & \mathrm{~T} & \mathrm{~T} & \mathrm{~T}\end{array}$ $\begin{array}{llllllll}0.2164033529507331 & 0.4457303361059829 & 0.2630829286001410 & \text { T } & \text { T } & \text { T }\end{array}$ $\begin{array}{lllllll}0.5581614066197580 & 0.4441259826017648 & 0.2633187266039544 & \mathrm{~T} & \mathrm{~T} & \mathrm{~T}\end{array}$ $\begin{array}{llllllll}0.8892466348925674 & 0.4448663300582069 & 0.2672400397372539 & \mathrm{~T} & \mathrm{~T} & \mathrm{~T}\end{array}$ $\begin{array}{llllllllll}0.2182326166180671 & 0.7741645511320467 & 0.2666262824704302 & \mathrm{~T} & \mathrm{~T} & \mathrm{~T}\end{array}$ $\begin{array}{llllllllll}0.5534671054439910 & 0.7763371945688036 & 0.2657150756983881 & \mathrm{~T} & \mathrm{~T} & \mathrm{~T}\end{array}$ $\begin{array}{llllllllll}0.8890250925352465 & 0.7769019827857860 & 0.2664033998911977 & \mathrm{~T} & \mathrm{~T} & \mathrm{~T}\end{array}$ $\begin{array}{llllllll}0.9948437201046559 & 0.9959777563969873 & 0.3517382432137072 & \text { T } & \text { T } & \text { T }\end{array}$ $\begin{array}{llllllll}0.3359843862957707 & 0.9959822160505551 & 0.3498883544570784 & \text { T } & \text { T } & \text { T }\end{array}$ $\begin{array}{llllllll}0.6653541717899776 & 0.9986626018550370 & 0.3490953257136969 & \mathrm{~T} & \mathrm{~T} & \mathrm{~T}\end{array}$ $\begin{array}{lllllllll}0.9935736476130420 & 0.3293737058484049 & 0.3540130100213965 & \mathrm{~T} & \mathrm{~T} & \mathrm{~T}\end{array}$ $\begin{array}{llllllll}0.3327135497530770 & 0.3383807152484827 & 0.3431273192806615 & \mathrm{~T} & \mathrm{~T} & \mathrm{~T}\end{array}$ $\begin{array}{lllllllllll}0.6629884243189098 & 0.3300592112767888 & 0.3518276733600922 & \mathrm{~T} & \mathrm{~T} & \mathrm{~T}\end{array}$ $\begin{array}{lllllllll}-0.0043425160718574 & 0.6698529504151302 & 0.3568212378598039 & \text { T } & \text { T } & \text { T }\end{array}$ $\begin{array}{llllllllll}0.3366925423771928 & 0.6676347293078732 & 0.3530429350671345 & \mathrm{~T} & \mathrm{~T} & \mathrm{~T}\end{array}$ $\begin{array}{llllllll}0.6642444268131792 & 0.6639343340313545 & 0.3497863560168522 & \mathrm{~T} & \mathrm{~T} & \mathrm{~T}\end{array}$ $\begin{array}{lllllllll}0.3320186768621581 & 0.2293676480123676 & 0.4494713401881902 & \mathrm{~T} & \mathrm{~T} & \mathrm{~T}\end{array}$ $\begin{array}{lllllllll}0.2271885861212594 & 0.4392906274623051 & 0.4097660960665513 & \mathrm{~T} & \mathrm{~T} & \mathrm{~T}\end{array}$ $\begin{array}{lllllllll}0.1274272498665911 & 0.9132561936378002 & 0.4135729059342196 & \mathrm{~T} & \mathrm{~T} & \mathrm{~T}\end{array}$ $\begin{array}{lllllllll}0.7540335426856951 & 0.5326592052211282 & 0.3972744612015613 & \mathrm{~T} & \mathrm{~T} & \mathrm{~T}\end{array}$

\section{1) $\mathrm{H}-\mathrm{Na}_{2} \mathrm{O}-\mathrm{Co}(111)-3 \times 3$}

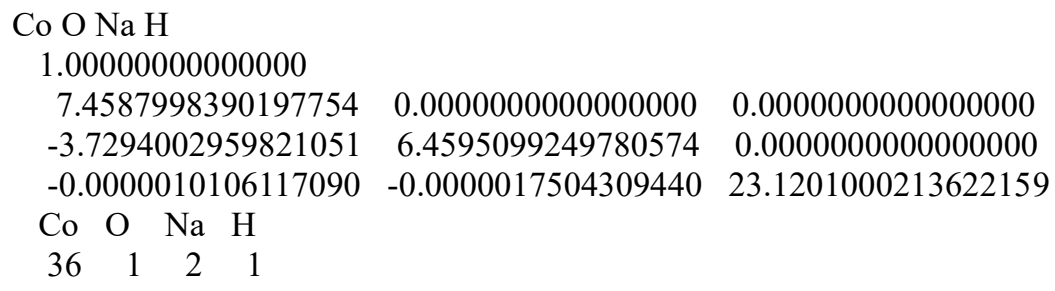

Selective dynamics

Direct

$\begin{array}{llllllll}0.0000353225881327 & 0.9999648942106703 & 0.0907110695049766 & F & F & F\end{array}$ $\begin{array}{llllllll}0.3333557427970177 & 0.0000001793936022 & 0.0907175141137770 & F & F & F\end{array}$ $\begin{array}{lllllll}0.6666917648932014 & 0.9999938437795421 & 0.0907267268767029 & F & F & F\end{array}$ $\begin{array}{lllllll}0.0000062578975388 & 0.3333084372988466 & 0.0907267268767029 & F & F & F\end{array}$ $\begin{array}{lllllllll}0.3333357007148621 & 0.3333426504206471 & 0.0907265971194704 & F & F & F\end{array}$ $\begin{array}{lllllllll}0.6666945969032270 & 0.3333051862794676 & 0.0907313116319486 & F & F & F\end{array}$ $\begin{array}{llllllll}0.9999998791701969 & 0.6666444062796870 & 0.0907175141137770 & F & F & F\end{array}$ $\begin{array}{llllllll}0.3333385397113773 & 0.6666614354387903 & 0.0907302303217534 & F & F & F\end{array}$ $\begin{array}{lllllllll}0.6666573639132167 & 0.6666642220269168 & 0.0907265971194704 & F & F & F\end{array}$ $\begin{array}{llllllll}0.1111175321760314 & 0.2222028177229376 & 0.1750651163386081 & F & F & F\end{array}$ $\begin{array}{llllllllll}0.4444257597051688 & 0.2222195372577005 & 0.1750655488626904 & F & F & F\end{array}$ 
$\begin{array}{llllllll}0.7777781372906531 & 0.2222218594174734 & 0.1750735938105805 & F & F & F\end{array}$ $\begin{array}{llllllll}0.1111123692640206 & 0.5555522552209098 & 0.1750674087162309 & F & F & F\end{array}$ $\begin{array}{llllllll}0.4444440697289167 & 0.5555561254847916 & 0.1750719502190776 & F & F & F\end{array}$ $\begin{array}{llllllll}0.7777804782179913 & 0.5555742383122606 & 0.1750655488626904 & F & F & F\end{array}$ $\begin{array}{lllllllll}0.1111199658994622 & 0.8888800192425492 & 0.1750494157144900 & F & F & F\end{array}$ $\begin{array}{llllllll}0.4444476042812084 & 0.8888872953412132 & 0.1750674087162309 & F & F & F\end{array}$ $\begin{array}{llllllll}0.7777970141657633 & 0.8888823414043898 & 0.1750651163386081 & F & F & F\end{array}$ $\begin{array}{llllllllll}0.2220336880034814 & 0.1103178370011476 & 0.2655455311724691 & \mathrm{~T} & \mathrm{~T} & \mathrm{~T}\end{array}$ $\begin{array}{llllllll}0.5545196097401827 & 0.1099351487842747 & 0.2652706876494403 & \text { T } & \text { T } & \text { T }\end{array}$ $\begin{array}{llllllll}0.8888093143999289 & 0.1084257349884131 & 0.2644440007831217 & \mathrm{~T} & \mathrm{~T} & \mathrm{~T}\end{array}$ $\begin{array}{llllllll}0.2242505714226629 & 0.4452187157969892 & 0.2657896014704487 & \mathrm{~T} & \mathrm{~T} & \mathrm{~T}\end{array}$ $\begin{array}{llllllll}0.5554704360603749 & 0.4439521219343788 & 0.2656342208764292 & \mathrm{~T} & \mathrm{~T} & \mathrm{~T}\end{array}$ $\begin{array}{llllllll}0.8889372104003841 & 0.4465409979669758 & 0.2639444387473621 & \mathrm{~T} & \mathrm{~T} & \mathrm{~T}\end{array}$ $\begin{array}{lllllll}0.2227663146891153 & 0.7774438039533232 & 0.2658277166235924 & \mathrm{~T} & \mathrm{~T} & \mathrm{~T}\end{array}$ $\begin{array}{lllllll}0.5541245980090254 & 0.7776000584814576 & 0.2660095512679364 & \mathrm{~T} & \mathrm{~T} & \mathrm{~T}\end{array}$ $\begin{array}{llllllll}0.8888663813522090 & 0.7776800842128224 & 0.2650154902384230 & \mathrm{~T} & \mathrm{~T} & \mathrm{~T}\end{array}$ $\begin{array}{lllllll}0.9989399003128685 & -0.0006280610299482 & 0.3497974240970271 & \mathrm{~T} & \mathrm{~T} & \mathrm{~T}\end{array}$ $\begin{array}{lllllll}0.3315620622894667 & 0.9981871764899126 & 0.3510007538373205 & \mathrm{~T} & \mathrm{~T} & \mathrm{~T}\end{array}$ $\begin{array}{lllllll}0.6705990469366677 & 0.0052990926475594 & 0.3511806651809586 & \mathrm{~T} & \mathrm{~T} & \mathrm{~T}\end{array}$ $\begin{array}{llllllll}0.9933789854474215 & 0.3308128488377471 & 0.3468777636870993 & \mathrm{~T} & \mathrm{~T} & \mathrm{~T}\end{array}$ $\begin{array}{lllllll}0.3332618234031243 & 0.3310121940015572 & 0.3521696650103656 & \mathrm{~T} & \mathrm{~T} & \mathrm{~T}\end{array}$ $\begin{array}{lllllll}0.6708812817444638 & 0.3305595303049425 & 0.3503563639212385 & \mathrm{~T} & \mathrm{~T} & \mathrm{~T}\end{array}$ $\begin{array}{llllllll}-0.0006316172099991 & 0.6644525754182483 & 0.3493852256552255 & \mathrm{~T} & \mathrm{~T} & \mathrm{~T}\end{array}$ $\begin{array}{llllllll}0.3307646249012560 & 0.6641973788539290 & 0.3543583296509578 & \mathrm{~T} & \mathrm{~T} & \mathrm{~T}\end{array}$ $\begin{array}{lllllll}0.6664458882876343 & 0.6694040445299623 & 0.3501074430284503 & \mathrm{~T} & \mathrm{~T} & \mathrm{~T}\end{array}$ $\begin{array}{lllllll}0.4514718611410282 & 0.5540878727318208 & 0.4102626316358868 & \mathrm{~T} & \mathrm{~T} & \mathrm{~T}\end{array}$ $\begin{array}{llllllll}0.1409595328448180 & 0.3595239767581421 & 0.4572753099306215 & \mathrm{~T} & \mathrm{~T} & \mathrm{~T}\end{array}$ $\begin{array}{lllllll}0.7446096333102815 & 0.5895126693954520 & 0.4576938552972054 & \mathrm{~T} & \mathrm{~T} & \mathrm{~T}\end{array}$ $\begin{array}{lllllll}0.5531782915113247 & 0.1122612188743686 & 0.3940216082450360 & \mathrm{~T} & \mathrm{~T} & \mathrm{~T}\end{array}$

\section{2) $\mathrm{CO}-\mathrm{Co}(111)-3 \times 3$}

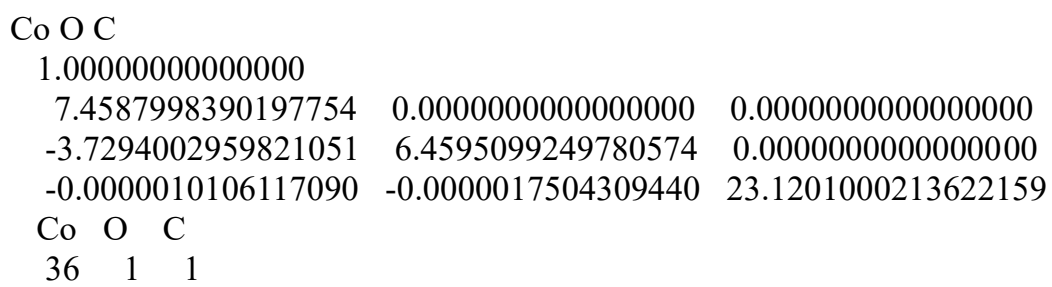

Selective dynamics

Direct

$\begin{array}{llllllll}0.0000353225881327 & 0.9999648942106703 & 0.0907110695049766 & F & F & F\end{array}$ $\begin{array}{llllllll}0.3333557427970177 & 0.0000001793936022 & 0.0907175141137770 & F & F & F\end{array}$ $\begin{array}{llllllll}0.6666917648932014 & 0.9999938437795421 & 0.0907267268767029 & F & F & F\end{array}$ $\begin{array}{lllllllll}0.0000062578975388 & 0.3333084372988466 & 0.0907267268767029 & F & F & F\end{array}$ $\begin{array}{llllllll}0.3333357007148621 & 0.3333426504206471 & 0.0907265971194704 & F & F & F\end{array}$ $\begin{array}{llllllll}0.6666945969032270 & 0.3333051862794676 & 0.0907313116319486 & F & F & F\end{array}$ $\begin{array}{lllllllll}0.9999998791701969 & 0.6666444062796870 & 0.0907175141137770 & F & F & F\end{array}$ $\begin{array}{lllllllll}0.3333385397113773 & 0.6666614354387903 & 0.0907302303217534 & F & F & F\end{array}$ $\begin{array}{lllllllll}0.6666573639132167 & 0.6666642220269168 & 0.0907265971194704 & F & F & F\end{array}$ $\begin{array}{llllllll}0.1111175321760314 & 0.2222028177229376 & 0.1750651163386081 & F & F & F\end{array}$ $\begin{array}{llllllll}0.4444257597051688 & 0.2222195372577005 & 0.1750655488626904 & F & F & F\end{array}$ $\begin{array}{llllllll}0.7777781372906531 & 0.2222218594174734 & 0.1750735938105805 & F & F & F\end{array}$ $\begin{array}{llllllll}0.1111123692640206 & 0.5555522552209098 & 0.1750674087162309 & F & F & F\end{array}$ 
$\begin{array}{llllllll}0.4444440697289167 & 0.5555561254847916 & 0.1750719502190776 & F & F & F\end{array}$ $\begin{array}{llllllll}0.7777804782179913 & 0.5555742383122606 & 0.1750655488626904 & F & F & F\end{array}$ $\begin{array}{llllllll}0.1111199658994622 & 0.8888800192425492 & 0.1750494157144900 & F & F & F\end{array}$ $\begin{array}{llllllll}0.4444476042812084 & 0.8888872953412132 & 0.1750674087162309 & F & F & F\end{array}$ $\begin{array}{llllllll}0.7777970141657633 & 0.8888823414043898 & 0.1750651163386081 & F & F & F\end{array}$ $\begin{array}{llllllll}0.2221036974284626 & 0.1108669016928796 & 0.2650251033425200 & \mathrm{~T} & \mathrm{~T} & \mathrm{~T}\end{array}$ $\begin{array}{llllllll}0.5554764457769095 & 0.1108803365706740 & 0.2650265186596194 & \mathrm{~T} & \mathrm{~T} & \mathrm{~T}\end{array}$ $\begin{array}{llllllll}0.8889069478731588 & 0.1110929363341530 & 0.2649879941267082 & \mathrm{~T} & \mathrm{~T} & \mathrm{~T}\end{array}$ $\begin{array}{llllllll}0.2220873552126460 & 0.4445307453312975 & 0.2650259249668122 & \mathrm{~T} & \mathrm{~T} & \mathrm{~T}\end{array}$ $\begin{array}{lllllll}0.5555638123215225 & 0.4444410809561343 & 0.2663685461318546 & \mathrm{~T} & \mathrm{~T} & \mathrm{~T}\end{array}$ $\begin{array}{llllllll}0.8891219341067715 & 0.4445257665880609 & 0.2650253595715871 & \mathrm{~T} & \mathrm{~T} & \mathrm{~T}\end{array}$ $\begin{array}{llllllll}0.2222231281630335 & 0.7777782381542686 & 0.2650931804688371 & \mathrm{~T} & \mathrm{~T} & \mathrm{~T}\end{array}$ $\begin{array}{llllllll}0.5554673009132628 & 0.7779130286603022 & 0.2650275140874026 & \mathrm{~T} & \mathrm{~T} & \mathrm{~T}\end{array}$ $\begin{array}{lllllll}0.8891326671982605 & 0.7779012885493886 & 0.2650259637089440 & \mathrm{~T} & \mathrm{~T} & \mathrm{~T}\end{array}$ $\begin{array}{llllllll}-0.0001400996809232 & 0.0001400943895795 & 0.3486062486709154 & \mathrm{~T} & \mathrm{~T} & \mathrm{~T}\end{array}$ $\begin{array}{lllllll}0.3322933912777389 & 0.9978779787815756 & 0.3493625904538315 & \mathrm{~T} & \mathrm{~T} & \mathrm{~T}\end{array}$ $\begin{array}{llllllll}0.6669540885494030 & 0.0001441053840270 & 0.3486042788051772 & \mathrm{~T} & \mathrm{~T} & \mathrm{~T}\end{array}$ $\begin{array}{lllllll}-0.0001456771256583 & 0.3330430722955081 & 0.3486032654043956 & \mathrm{~T} & \mathrm{~T} & \mathrm{~T}\end{array}$ $\begin{array}{lllllll}0.3311943308095472 & 0.3322629339010165 & 0.3521607889053422 & \mathrm{~T} & \mathrm{~T} & \mathrm{~T}\end{array}$ $\begin{array}{lllllllll}0.6677402652691588 & 0.3322586939784946 & 0.3521635815894781 & \mathrm{~T} & \mathrm{~T} & \mathrm{~T}\end{array}$ $\begin{array}{llllllll}0.0021166883284463 & 0.6677085770723037 & 0.3493629441061497 & \mathrm{~T} & \mathrm{~T} & \mathrm{~T}\end{array}$ $\begin{array}{llllllll}0.3322750211082653 & 0.6677059568972596 & 0.3493655412845165 & \mathrm{~T} & \mathrm{~T} & \mathrm{~T}\end{array}$ $\begin{array}{llllllll}0.6677389575800685 & 0.6688122093019238 & 0.3521631872160244 & \mathrm{~T} & \mathrm{~T} & \mathrm{~T}\end{array}$ $\begin{array}{lllllllll}0.5555440019058292 & 0.4444667002070210 & 0.4616508139109228 & \mathrm{~T} & \mathrm{~T} & \mathrm{~T}\end{array}$ $\begin{array}{lllllll}0.5555585436222646 & 0.4444471693398092 & 0.4098158261853002 & \mathrm{~T} & \mathrm{~T} & \mathrm{~T}\end{array}$

\section{3) $\mathrm{CO}-\mathrm{PrO}_{2}-\mathrm{Co}(111)-3 \times 3$}

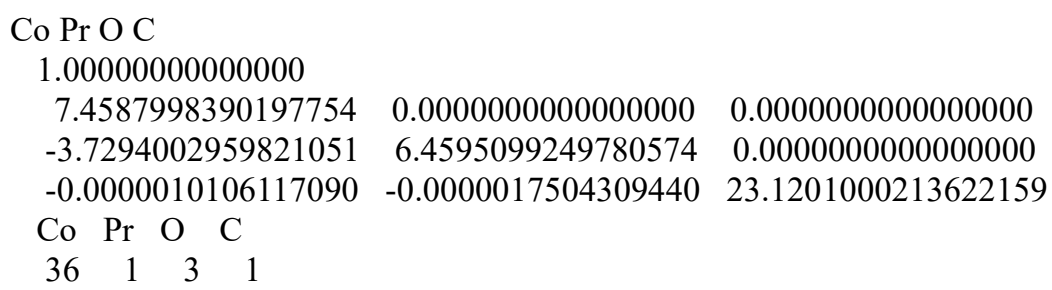

Selective dynamics

Direct

$\begin{array}{lllllll}0.0000353225881327 & 0.9999648942106703 & 0.0907110695049766 & F & F & F\end{array}$ $\begin{array}{llllllll}0.3333557427970177 & 0.0000001793936022 & 0.0907175141137770 & F & F & F\end{array}$ $\begin{array}{llllllll}0.6666917648932014 & 0.9999938437795421 & 0.0907267268767029 & F & F & F\end{array}$ $\begin{array}{lllllllll}0.0000062578975388 & 0.3333084372988466 & 0.0907267268767029 & F & F & F\end{array}$ $\begin{array}{lllllllll}0.3333357007148621 & 0.3333426504206471 & 0.0907265971194704 & F & F & F\end{array}$ $\begin{array}{lllllllll}0.6666945969032270 & 0.3333051862794676 & 0.0907313116319486 & F & F & F\end{array}$ $\begin{array}{llllll}0.9999998791701969 & 0.6666444062796870 & 0.0907175141137770 & F & F & F\end{array}$ $\begin{array}{llllllll}0.3333385397113773 & 0.6666614354387903 & 0.0907302303217534 & F & F & F\end{array}$ $\begin{array}{lllllllll}0.6666573639132167 & 0.6666642220269168 & 0.0907265971194704 & F & F & F\end{array}$ $\begin{array}{llllllll}0.1111175321760314 & 0.2222028177229376 & 0.1750651163386081 & F & F & F\end{array}$ $\begin{array}{lllllllll}0.4444257597051688 & 0.2222195372577005 & 0.1750655488626904 & F & F & F\end{array}$ $\begin{array}{lllllllll}0.7777781372906531 & 0.2222218594174734 & 0.1750735938105805 & F & F & F\end{array}$ $\begin{array}{lllllllll}0.1111123692640206 & 0.5555522552209098 & 0.1750674087162309 & F & F & F\end{array}$ $\begin{array}{llllllll}0.4444440697289167 & 0.5555561254847916 & 0.1750719502190776 & F & F & F\end{array}$ $\begin{array}{llllllll}0.7777804782179913 & 0.5555742383122606 & 0.1750655488626904 & F & F & F\end{array}$ $\begin{array}{llllllll}0.1111199658994622 & 0.8888800192425492 & 0.1750494157144900 & F & F & F\end{array}$ $\begin{array}{llllllll}0.4444476042812084 & 0.8888872953412132 & 0.1750674087162309 & F & F & F\end{array}$ 
$\begin{array}{llllllll}0.7777970141657633 & 0.8888823414043898 & 0.1750651163386081 & F & F & F\end{array}$ $\begin{array}{llllllllll}0.2246204323930829 & 0.1133391550036773 & 0.2658276030001413 & \mathrm{~T} & \mathrm{~T} & \mathrm{~T}\end{array}$ $\begin{array}{lllllllll}0.5541713353262103 & 0.1100014613146186 & 0.2640123926539331 & \mathrm{~T} & \mathrm{~T} & \mathrm{~T}\end{array}$ $\begin{array}{lllllllll}0.8898993497032982 & 0.1123094112548517 & 0.2645471850347881 & \mathrm{~T} & \mathrm{~T} & \mathrm{~T}\end{array}$ $\begin{array}{llllllll}0.2210885426706755 & 0.4458598360629237 & 0.26457940744435593 & \mathrm{~T} & \mathrm{~T} & \mathrm{~T}\end{array}$ $\begin{array}{llllllllll}0.5576773693763727 & 0.4451470709112306 & 0.2649468187374439 & \mathrm{~T} & \mathrm{~T} & \mathrm{~T}\end{array}$ $\begin{array}{llllllll}0.8913436892489106 & 0.4461092891940112 & 0.2650190181547976 & \mathrm{~T} & \mathrm{~T} & \mathrm{~T}\end{array}$ $\begin{array}{llllllll}0.2215643484408887 & 0.7778075551209848 & 0.2666735642558533 & \mathrm{~T} & \mathrm{~T} & \mathrm{~T}\end{array}$ $\begin{array}{llllllllll}0.5536610888178682 & 0.7764350927744694 & 0.2649301268503573 & \mathrm{~T} & \mathrm{~T} & \mathrm{~T}\end{array}$ $\begin{array}{llllllll}0.8905342548718762 & 0.7764208964470659 & 0.2649190301361435 & \mathrm{~T} & \mathrm{~T} & \mathrm{~T}\end{array}$ $\begin{array}{lllllllll}0.9939195788799134 & 0.9955037388540243 & 0.3490326042370613 & \mathrm{~T} & \mathrm{~T} & \mathrm{~T}\end{array}$ $\begin{array}{llllllll}0.3354125956071713 & 0.9979449343467273 & 0.3522763554554164 & \mathrm{~T} & \mathrm{~T} & \mathrm{~T}\end{array}$ $\begin{array}{llllllllll}0.6653548787904541 & 0.9982830947628608 & 0.3479013818888871 & \mathrm{~T} & \mathrm{~T} & \mathrm{~T}\end{array}$ $\begin{array}{lllllllllll}0.9904870576075824 & 0.3281736009348064 & 0.3511469355499036 & \mathrm{~T} & \mathrm{~T} & \mathrm{~T}\end{array}$ $\begin{array}{llllllll}0.3412677865475350 & 0.3416720471603109 & 0.3519131903318257 & \mathrm{~T} & \mathrm{~T} & \mathrm{~T}\end{array}$ $\begin{array}{llllllllll}0.6688227605838196 & 0.3295892972094258 & 0.3506555156975117 & \mathrm{~T} & \mathrm{~T} & \mathrm{~T}\end{array}$ $\begin{array}{llllllllll}0.9986773813777425 & 0.6704056590253886 & 0.3530409060552684 & \mathrm{~T} & \mathrm{~T} & \mathrm{~T}\end{array}$ $\begin{array}{llllllllll}0.3373299384665452 & 0.6726091769618736 & 0.3518221686071695 & \mathrm{~T} & \mathrm{~T} & \mathrm{~T}\end{array}$ $\begin{array}{lllllllllll}0.6689453437808269 & 0.6694519349845311 & 0.3518799883867835 & \mathrm{~T} & \mathrm{~T} & \mathrm{~T}\end{array}$ $\begin{array}{llllllllll}0.1956288523832697 & 0.1891781418754368 & 0.4582191725521584 & \mathrm{~T} & \mathrm{~T} & \mathrm{~T}\end{array}$ $\begin{array}{llllllllll}0.2009808186302912 & 0.4506353041051009 & 0.4088110330178136 & \mathrm{~T} & \mathrm{~T} & \mathrm{~T}\end{array}$ $\begin{array}{lllllllll}0.1116329003371559 & 0.8936502927781822 & 0.4113691139093410 & \mathrm{~T} & \mathrm{~T} & \mathrm{~T}\end{array}$ $\begin{array}{lllllllll}0.5624823659074319 & 0.4334785591848711 & 0.4617079351304048 & \mathrm{~T} & \mathrm{~T} & \mathrm{~T}\end{array}$ $\begin{array}{lllllllllll}0.5761598656011695 & 0.4460733485274896 & 0.4065385691988560 & \mathrm{~T} & \mathrm{~T} & \mathrm{~T}\end{array}$

14) $\mathrm{CO}-\mathrm{Na}_{2} \mathrm{O}-\mathrm{Co}(111)-3 \times 3$

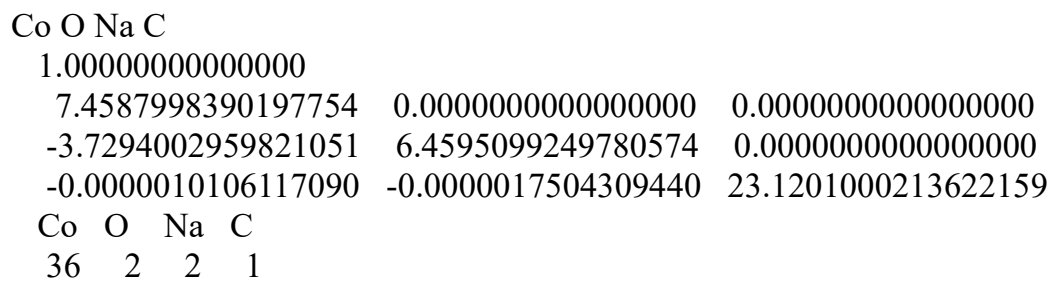

Selective dynamics

Direct

$\begin{array}{llllllll}0.0000353225881327 & 0.9999648942106703 & 0.0907110695049766 & F & F & F\end{array}$ $\begin{array}{llllllll}0.3333557427970177 & 0.0000001793936022 & 0.0907175141137770 & \mathrm{~F} & \mathrm{~F} & \mathrm{~F}\end{array}$ $\begin{array}{llllllll}0.6666917648932014 & 0.99999384377795421 & 0.0907267268767029 & F & F & F\end{array}$ $\begin{array}{lllllll}0.0000062578975388 & 0.33330843729884666 & 0.0907267268767029 & F & F & F\end{array}$ $\begin{array}{llllllll}0.3333357007148621 & 0.3333426504206471 & 0.0907265971194704 & F & F & F\end{array}$ $\begin{array}{llllllllll}0.6666945969032270 & 0.3333051862794676 & 0.0907313116319486 & F & F & F\end{array}$ $\begin{array}{lllllllll}0.9999998791701969 & 0.6666444062796870 & 0.0907175141137770 & F & F & F\end{array}$ $\begin{array}{llllllll}0.3333385397113773 & 0.6666614354387903 & 0.0907302303217534 & F & F & F\end{array}$ $\begin{array}{lllllllll}0.6666573639132167 & 0.6666642220269168 & 0.0907265971194704 & F & F & F\end{array}$ $\begin{array}{llllllll}0.1111175321760314 & 0.2222028177229376 & 0.1750651163386081 & F & F & F\end{array}$ $\begin{array}{lllllllll}0.4444257597051688 & 0.2222195372577005 & 0.1750655488626904 & F & F & F\end{array}$ $\begin{array}{llllllll}0.7777781372906531 & 0.2222218594174734 & 0.1750735938105805 & F & F & F\end{array}$ $\begin{array}{lllllllll}0.1111123692640206 & 0.5555522552209098 & 0.1750674087162309 & \mathrm{~F} & \mathrm{~F} & \mathrm{~F}\end{array}$ $\begin{array}{llllllll}0.4444440697289167 & 0.55555612548477916 & 0.1750719502190776 & F & F & F\end{array}$ $\begin{array}{llllllll}0.7777804782179913 & 0.5555742383122606 & 0.1750655488626904 & F & F & F\end{array}$ $\begin{array}{lllllll}0.1111199658994622 & 0.8888800192425492 & 0.1750494157144900 & F & F & F\end{array}$ $\begin{array}{llllllll}0.4444476042812084 & 0.8888872953412132 & 0.1750674087162309 & F & F & F\end{array}$ $\begin{array}{llllllll}0.7777970141657633 & 0.8888823414043898 & 0.1750651163386081 & F & F & F\end{array}$ 
$\begin{array}{lllllll}0.2226599004348977 & 0.1121061546981159 & 0.2653456600965885 & \mathrm{~T} & \mathrm{~T} & \mathrm{~T}\end{array}$ $\begin{array}{llllllll}0.5547213267799483 & 0.1105638157776499 & 0.2646009590223369 & \mathrm{~T} & \mathrm{~T} & \mathrm{~T}\end{array}$ $\begin{array}{llllllll}0.8888696872656416 & 0.1102462204533707 & 0.2653687238078457 & \mathrm{~T} & \mathrm{~T} & \mathrm{~T}\end{array}$ $\begin{array}{llllllll}0.2240607022663711 & 0.4443289132927175 & 0.2661265318169739 & \mathrm{~T} & \mathrm{~T} & \mathrm{~T}\end{array}$ $\begin{array}{llllllll}0.5536989755726143 & 0.4427655013792817 & 0.2654364956850360 & \mathrm{~T} & \mathrm{~T} & \mathrm{~T}\end{array}$ $\begin{array}{llllllll}0.8886873497797644 & 0.4459019154056134 & 0.2637844648571075 & \mathrm{~T} & \mathrm{~T} & \mathrm{~T}\end{array}$ $\begin{array}{lllllll}0.2224125151728259 & 0.7773518841563372 & 0.2651039622049164 & \mathrm{~T} & \mathrm{~T} & \mathrm{~T}\end{array}$ $\begin{array}{lllllll}0.5553685795652969 & 0.7770575004983036 & 0.2653480976954286 & \mathrm{~T} & \mathrm{~T} & \mathrm{~T}\end{array}$ $\begin{array}{lllllll}0.8886110753474302 & 0.7776884573663773 & 0.2647905801946258 & \mathrm{~T} & \mathrm{~T} & \mathrm{~T}\end{array}$ $\begin{array}{lllllll}0.0016099844740726 & -0.0009435951152835 & 0.3519168862307597 & \mathrm{~T} & \mathrm{~T} & \mathrm{~T}\end{array}$ $\begin{array}{lllllll}0.3334868960320312 & 0.9993515959305129 & 0.3488473430394419 & \mathrm{~T} & \mathrm{~T} & \mathrm{~T}\end{array}$ $\begin{array}{llllllll}0.6671829757792229 & 0.0036906634778825 & 0.3521825937468550 & \mathrm{~T} & \mathrm{~T} & \mathrm{~T}\end{array}$ $\begin{array}{lllllll}0.9949314558822192 & 0.3332568193041241 & 0.3503182918049295 & \mathrm{~T} & \mathrm{~T} & \mathrm{~T}\end{array}$ $\begin{array}{llllllll}0.3389614803561818 & 0.3341391424361143 & 0.3539099499186197 & \mathrm{~T} & \mathrm{~T} & \mathrm{~T}\end{array}$ $\begin{array}{llllllll}0.6691963246859348 & 0.3321083970320101 & 0.3486177392686298 & \mathrm{~T} & \mathrm{~T} & \mathrm{~T}\end{array}$ $\begin{array}{llllllll}0.0015012880888389 & 0.6660005678259459 & 0.3493023254699507 & \mathrm{~T} & \mathrm{~T} & \mathrm{~T}\end{array}$ $\begin{array}{llllllll}0.3358397186949341 & 0.6701436224220975 & 0.3522704955085173 & \mathrm{~T} & \mathrm{~T} & \mathrm{~T}\end{array}$ $\begin{array}{llllllll}0.6661628261162766 & 0.6606347645985137 & 0.3495311303091539 & \mathrm{~T} & \mathrm{~T} & \mathrm{~T}\end{array}$ $\begin{array}{llllllll}0.4532359436579617 & 0.5638892597075303 & 0.4103487418522269 & \mathrm{~T} & \mathrm{~T} & \mathrm{~T}\end{array}$ $\begin{array}{lllllll}0.8963756332869324 & 0.1156894208806303 & 0.4616256752648365 & \mathrm{~T} & \mathrm{~T} & \mathrm{~T}\end{array}$ $\begin{array}{llllllll}0.1564103136326972 & 0.4641062300338658 & 0.4611549521352474 & \mathrm{~T} & \mathrm{~T} & \mathrm{~T}\end{array}$ $\begin{array}{llllllll}0.7644123521711568 & 0.7602125264291260 & 0.4610694240340514 & \mathrm{~T} & \mathrm{~T} & \mathrm{~T}\end{array}$ $\begin{array}{llllllll}0.8952425055837765 & 0.1199411817164053 & 0.4063412535039359 & \mathrm{~T} & \mathrm{~T} & \mathrm{~T}\end{array}$

15) $\mathrm{H}-\mathrm{Co}(211)-1 \times 3$

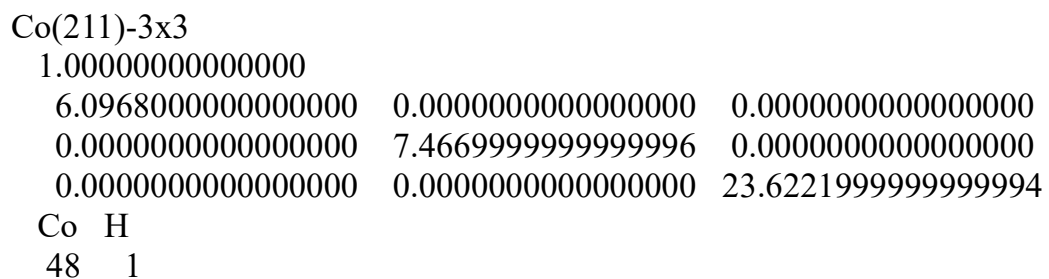

Selective dynamics

Direct

$\begin{array}{lllllllll}0.0039768403096687 & 0.1666663988214836 & 0.9745822997011260 & \mathrm{~F} & \mathrm{~F} & \mathrm{~F}\end{array}$ $\begin{array}{llllllll}0.0039765122687285 & 0.5000000000000000 & 0.9745820457027676 & F & F & F\end{array}$ $\begin{array}{llllllll}0.0039768403096687 & 0.8333336011785164 & 0.9745822997011260 & F & F & F\end{array}$ $\begin{array}{llllllll}0.3315888662905095 & 0.0000000000000000 & 0.0034278771663949 & F & F & F\end{array}$ $\begin{array}{llllllll}0.3315855858811148 & 0.3333350743270387 & 0.0034291471581795 & F & F & F\end{array}$ $\begin{array}{lllllllll}0.3315855858811148 & 0.6666647917503710 & 0.0034291471581795 & \mathrm{~F} & \mathrm{~F} & \mathrm{~F}\end{array}$ $\begin{array}{lllllllll}0.6664853693741009 & 0.1666655952859273 & 0.0285661792720404 & F & F & F\end{array}$ $\begin{array}{lllllllll}0.6664884857630256 & 0.5000000000000000 & 0.0285654596100287 & F & F & F\end{array}$ $\begin{array}{llllllll}0.6664853693741009 & 0.8333344047140727 & 0.0285661792720404 & F & F & F\end{array}$ $\begin{array}{lllllllll}0.9983825941477491 & 0.0000000000000000 & 0.0609119811025209 & F & F & F\end{array}$ $\begin{array}{llllllll}0.9983791497178842 & 0.3333345386366702 & 0.0609079171288016 & F & F & F\end{array}$ $\begin{array}{lllllll}0.9983791497178842 & 0.6666654613633298 & 0.0609079171288016 & F & F & F\end{array}$ $\begin{array}{lllllllll}0.3314264860254568 & 0.1666650595955517 & 0.0918800111759310 & F & F & F\end{array}$ $\begin{array}{lllllllllll}0.3314281262301506 & 0.5000000000000000 & 0.0918805615057039 & \mathrm{~F} & \mathrm{~F} & \mathrm{~F}\end{array}$ $\begin{array}{llllllll}0.3314264860254568 & 0.8333349404044483 & 0.0918800111759310 & F & F & F\end{array}$ $\begin{array}{lllllll}0.6654751673008761 & 0.0000000000000000 & 0.1205095207050988 & F & F & F\end{array}$ $\begin{array}{lllllllll}0.6654631938065876 & 0.3333330654881479 & 0.1205040597404121 & F & F & F\end{array}$ $\begin{array}{llllllll}0.6654631938065876 & 0.6666669345118521 & 0.1205040597404121 & F & F & F\end{array}$ $\begin{array}{llllllll}0.0009121178323070 & 0.1666669345118521 & 0.1526111031148645 & F & F & F\end{array}$ 
$\begin{array}{lllllll}0.0009114617504267 & 0.5000000000000000 & 0.1526112301140472 & \mathrm{~F} & \mathrm{~F} & \mathrm{~F}\end{array}$ $\begin{array}{lllllllll}0.0009121178323070 & 0.8333330654881479 & 0.1526111031148645 & \mathrm{~F} & \mathrm{~F} & \mathrm{~F}\end{array}$ $\begin{array}{llllllllll}0.3340214538774404 & 0.0000000000000000 & 0.1833969740329024 & F & F & F\end{array}$ $\begin{array}{llllllllll}0.3340229300616713 & 0.3333327976429601 & 0.1833968470337197 & F & F & F\end{array}$ $\begin{array}{lllllllll}0.3340229300616713 & 0.6666672023570399 & 0.1833968470337197 & F & F & F\end{array}$ $\begin{array}{lllllllllll}0.6659075478749005 & 0.1667425763836577 & 0.2120608371868066 & \mathrm{~T} & \mathrm{~T} & \mathrm{~T}\end{array}$ $\begin{array}{llllllll}0.6654222140421522 & 0.4999804391611274 & 0.2120747318145073 & \mathrm{~T} & \mathrm{~T} & \mathrm{~T}\end{array}$ $\begin{array}{llllllll}0.6659060550970877 & 0.8332131059645395 & 0.2120587887988211 & \mathrm{~T} & \mathrm{~T} & \mathrm{~T}\end{array}$ $\begin{array}{lllllll}0.9993331675290085 & 0.0000025241197139 & 0.2425556302596794 & \mathrm{~T} & \mathrm{~T} & \mathrm{~T}\end{array}$ $\begin{array}{lllllll}0.9982086379092424 & 0.3333517232772652 & 0.2426471252536520 & \mathrm{~T} & \mathrm{~T} & \mathrm{~T}\end{array}$ $\begin{array}{lllllllll}0.9982071957486578 & 0.6666102478270592 & 0.2426436655430493 & \mathrm{~T} & \mathrm{~T} & \mathrm{~T}\end{array}$ $\begin{array}{lllllllll}0.3346643569559713 & 0.1666807671243641 & 0.2747684054115404 & \mathrm{~T} & \mathrm{~T} & \mathrm{~T}\end{array}$ $\begin{array}{lllllll}0.3337915709990743 & 0.4999831682990099 & 0.2749892740702782 & \text { T } & \text { T } & \text { T }\end{array}$ $\begin{array}{llllllll}0.3346772120115538 & 0.8332931158111589 & 0.2747723822142937 & \mathrm{~T} & \mathrm{~T} & \mathrm{~T}\end{array}$ $\begin{array}{lllllllll}0.6691866228884117 & -0.0000409899969991 & 0.3033163937320021 & \mathrm{~T} & \mathrm{~T} & \mathrm{~T}\end{array}$ $\begin{array}{lllllllll}0.6674888440355041 & 0.3339969845417470 & 0.3037061707485622 & \mathrm{~T} & \mathrm{~T} & \mathrm{~T}\end{array}$ $\begin{array}{lllllllll}0.6674732661394517 & 0.6659386861055385 & 0.3037262996008369 & \mathrm{~T} & \mathrm{~T} & \mathrm{~T}\end{array}$ $\begin{array}{llllllllll}0.0018387078492426 & 0.1662691251799880 & 0.3340110045415151 & \mathrm{~T} & \mathrm{~T} & \mathrm{~T}\end{array}$ $\begin{array}{llllllll}0.0003819898157567 & 0.4999423300563042 & 0.3343458796982838 & \mathrm{~T} & \mathrm{~T} & \mathrm{~T}\end{array}$ $\begin{array}{lllllllllll}0.0018506325352221 & 0.8336872350016312 & 0.3339937148428434 & \mathrm{~T} & \mathrm{~T} & \mathrm{~T}\end{array}$ $\begin{array}{llllllllll}0.3328946935284433 & -0.0000211635235944 & 0.3663192053489376 & \mathrm{~T} & \mathrm{~T} & \mathrm{~T}\end{array}$ $\begin{array}{llllllll}0.3334100934074803 & 0.3332126409893287 & 0.3666987885690342 & \mathrm{~T} & \mathrm{~T} & \mathrm{~T}\end{array}$ $\begin{array}{llllllllll}0.3334141418636439 & 0.6667322570659311 & 0.3667085494275082 & \mathrm{~T} & \mathrm{~T} & \mathrm{~T}\end{array}$ $\begin{array}{lllllllll}0.6679883450717899 & 0.1656694592774273 & 0.3916581283242920 & \mathrm{~T} & \mathrm{~T} & \mathrm{~T}\end{array}$ $\begin{array}{llllllll}0.6676555225948456 & 0.4999024987587957 & 0.3939746017344007 & \mathrm{~T} & \mathrm{~T} & \mathrm{~T}\end{array}$ $\begin{array}{llllllll}0.6679396612973107 & 0.8341522832419273 & 0.3916580213788689 & \mathrm{~T} & \mathrm{~T} & \mathrm{~T}\end{array}$ $\begin{array}{lllllllll}0.9974811386156500 & -0.0001728302591857 & 0.4198030710211847 & \text { T } & \text { T } & \text { T }\end{array}$ $\begin{array}{llllllll}0.9952222112097231 & 0.3318765641747736 & 0.4210868539526317 & \mathrm{~T} & \mathrm{~T} & \mathrm{~T}\end{array}$ $\begin{array}{lllllllll}0.9951967524766275 & 0.6679774903050043 & 0.4210847767529043 & \mathrm{~T} & \mathrm{~T} & \mathrm{~T}\end{array}$ $\begin{array}{lllllllll}0.8464472131059547 & 0.4998689792134908 & 0.4547233256787429 & \mathrm{~T} & \mathrm{~T} & \mathrm{~T}\end{array}$

16) $\mathrm{H}-\mathrm{PrO}_{2}-\mathrm{Co}(211)-1 \times 3$

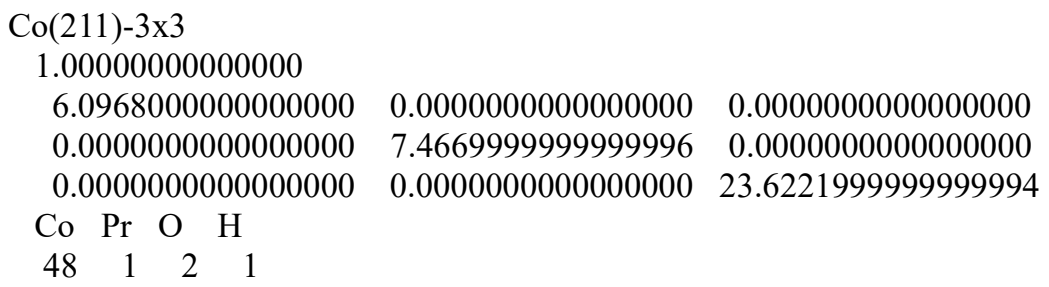

Selective dynamics

Direct

$\begin{array}{llllllll}0.0039768403096687 & 0.1666663988214836 & 0.9745822997011260 & F & F & F\end{array}$ $\begin{array}{llllllll}0.0039765122687285 & 0.5000000000000000 & 0.9745819610366553 & F & F & F\end{array}$ $\begin{array}{llllllll}0.0039768403096687 & 0.8333336011785164 & 0.9745822997011260 & F & F & F\end{array}$ $\begin{array}{lllllll}0.3315888662905095 & 0.0000000000000000 & 0.0034278771663949 & F & F & F\end{array}$ $\begin{array}{llllllll}0.3315855858811148 & 0.3333350743270387 & 0.0034291471581795 & F & F & F\end{array}$ $\begin{array}{llllllll}0.3315855858811148 & 0.6666647917503710 & 0.0034291471581795 & F & F & F\end{array}$ $\begin{array}{llllllll}0.6664853693741009 & 0.1666655952859273 & 0.0285661792720404 & F & F & F\end{array}$ $\begin{array}{llllllll}0.6664884857630256 & 0.5000000000000000 & 0.0285654596100287 & F & F & F\end{array}$ $\begin{array}{llllllll}0.6664853693741009 & 0.8333344047140727 & 0.0285661792720404 & F & F & F\end{array}$ $\begin{array}{lllllllll}0.9983825941477491 & 0.0000000000000000 & 0.0609119811025209 & F & F & F\end{array}$ $\begin{array}{lllllll}0.9983791497178842 & 0.33333453866366702 & 0.0609079171288016 & F & F & F\end{array}$ $\begin{array}{llllllll}0.9983791497178842 & 0.6666654613633298 & 0.0609079171288016 & F & F & F\end{array}$ 
$\begin{array}{llllllll}0.3314264860254568 & 0.1666650595955517 & 0.0918800111759310 & F & F & F\end{array}$ $\begin{array}{llllllll}0.3314281262301506 & 0.5000000000000000 & 0.0918805615057039 & \mathrm{~F} & \mathrm{~F} & \mathrm{~F}\end{array}$ $\begin{array}{llllllll}0.3314264860254568 & 0.8333349404044483 & 0.0918800111759310 & F & F & F\end{array}$ $\begin{array}{llllllll}0.6654751673008761 & 0.0000000000000000 & 0.1205095207050988 & F & F & F\end{array}$ $\begin{array}{llllllll}0.6654631938065876 & 0.33333330654881479 & 0.1205040597404121 & F & F & F\end{array}$ $\begin{array}{llllllll}0.6654631938065876 & 0.6666669345118521 & 0.1205040597404121 & F & F & F\end{array}$ $\begin{array}{lllllll}0.0009121178323070 & 0.1666669345118521 & 0.1526111031148645 & F & F & F\end{array}$ $\begin{array}{lllllll}0.0009114617504267 & 0.5000000000000000 & 0.1526112301140472 & F & F & F\end{array}$ $\begin{array}{llllllll}0.0009121178323070 & 0.8333330654881479 & 0.1526111031148645 & \mathrm{~F} & \mathrm{~F} & \mathrm{~F}\end{array}$ $\begin{array}{llllllll}0.3340214538774404 & 0.0000000000000000 & 0.1833969740329024 & F & F & F\end{array}$ $\begin{array}{llllllll}0.3340229300616713 & 0.3333327976429601 & 0.1833968470337197 & F & F & F\end{array}$ $\begin{array}{llllllll}0.3340229300616713 & 0.6666672023570399 & 0.1833968470337197 & F & F & F\end{array}$ $\begin{array}{llllllll}0.6652067687614618 & 0.1660260622257642 & 0.2117581642129120 & \mathrm{~T} & \mathrm{~T} & \mathrm{~T}\end{array}$ $\begin{array}{lllllllllll}0.6666112268661125 & 0.4996458227400032 & 0.2119678488169355 & \mathrm{~T} & \mathrm{~T} & \mathrm{~T}\end{array}$ $\begin{array}{llllllll}0.6659634727998642 & 0.8339010246993521 & 0.2119254963882900 & \mathrm{~T} & \mathrm{~T} & \mathrm{~T}\end{array}$ $\begin{array}{lllllllllll}0.9965483728025958 & 0.0006530877129741 & 0.2431953111975689 & \mathrm{~T} & \mathrm{~T} & \mathrm{~T}\end{array}$ $\begin{array}{lllllllll}-0.0001394948403334 & 0.3335991062127469 & 0.2428770173288116 & \mathrm{~T} & \mathrm{~T} & \mathrm{~T}\end{array}$ $\begin{array}{llllllll}-0.0000214143935374 & 0.6659671088440241 & 0.2427545259357554 & \mathrm{~T} & \mathrm{~T} & \mathrm{~T}\end{array}$ $\begin{array}{lllllllll}0.3325994222227879 & 0.1666639429315361 & 0.2755584668379062 & \mathrm{~T} & \mathrm{~T} & \mathrm{~T}\end{array}$ $\begin{array}{llllllll}0.3357474430119203 & 0.4982792723513034 & 0.2754832091969216 & \mathrm{~T} & \mathrm{~T} & \mathrm{~T}\end{array}$ $\begin{array}{lllllll}0.3319361501426492 & 0.8343230961211812 & 0.2739344005498196 & \mathrm{~T} & \mathrm{~T} & \mathrm{~T}\end{array}$ $\begin{array}{llllllll}0.6646240618638403 & 0.0004562453873235 & 0.3035652748013982 & \mathrm{~T} & \mathrm{~T} & \mathrm{~T}\end{array}$ $\begin{array}{lllllllll}0.6700510693230065 & 0.3287759840297700 & 0.3020667889191338 & \mathrm{~T} & \mathrm{~T} & \mathrm{~T}\end{array}$ $\begin{array}{llllllllll}0.6658343915442785 & 0.6679099398123817 & 0.3023237497390294 & \mathrm{~T} & \mathrm{~T} & \mathrm{~T}\end{array}$ $\begin{array}{llllllll}0.0002688444054577 & 0.1678067225182660 & 0.3347068440706438 & \mathrm{~T} & \mathrm{~T} & \mathrm{~T}\end{array}$ $\begin{array}{lllllllll}0.0044527745431080 & 0.4981992004458877 & 0.3347601341413541 & \mathrm{~T} & \mathrm{~T} & \mathrm{~T}\end{array}$ $\begin{array}{llllllllll}-0.0031084668870038 & 0.8312950923828641 & 0.3344620382393557 & \mathrm{~T} & \mathrm{~T} & \mathrm{~T}\end{array}$ $\begin{array}{lllllllll}0.3286362651398992 & -0.0042159488229669 & 0.3660650358483465 & \text { T } & \text { T } & \text { T }\end{array}$ $\begin{array}{lllllllll}0.3268795551249948 & 0.3304142199567579 & 0.3692866422545333 & \mathrm{~T} & \mathrm{~T} & \mathrm{~T}\end{array}$ $\begin{array}{llllllll}0.3277139890133021 & 0.6735579372191476 & 0.3641969911948855 & \mathrm{~T} & \mathrm{~T} & \mathrm{~T}\end{array}$ $\begin{array}{llllllll}0.6724902053901375 & 0.1621674089924025 & 0.3956328097408930 & \mathrm{~T} & \mathrm{~T} & \mathrm{~T}\end{array}$ $\begin{array}{llllllll}0.6713675508853488 & 0.4992180128120329 & 0.3870350954848259 & \mathrm{~T} & \mathrm{~T} & \mathrm{~T}\end{array}$ $\begin{array}{llllllll}0.6634329175548970 & 0.8348962500450262 & 0.3932780466450750 & \mathrm{~T} & \mathrm{~T} & \mathrm{~T}\end{array}$ $\begin{array}{lllllll}0.9958715053244804 & -0.0033237241002427 & 0.4204812411721088 & \mathrm{~T} & \mathrm{~T} & \mathrm{~T}\end{array}$ $\begin{array}{llllllll}-0.0000670470059774 & 0.3370599588476814 & 0.4259017916959014 & \mathrm{~T} & \mathrm{~T} & \mathrm{~T}\end{array}$ $\begin{array}{llllllll}0.9764519018850490 & 0.6678558346326093 & 0.4249906658215404 & \mathrm{~T} & \mathrm{~T} & \mathrm{~T}\end{array}$ $\begin{array}{lllllll}0.4035714243168471 & 0.5905800902576853 & 0.4824582666174612 & \mathrm{~T} & \mathrm{~T} & \mathrm{~T}\end{array}$ $\begin{array}{llllllll}0.5193082485350469 & 0.3394406089636171 & 0.4411547837462604 & \mathrm{~T} & \mathrm{~T} & \mathrm{~T}\end{array}$ $\begin{array}{llllllll}0.0565943590987361 & 0.5011089377371946 & 0.4852147774507706 & \text { T } & \text { T } & \text { T }\end{array}$ $\begin{array}{llllllllll}0.8176659049862471 & 0.8417249953457976 & 0.4550520341413495 & \mathrm{~T} & \mathrm{~T} & \mathrm{~T}\end{array}$

17) $\mathrm{H}-\mathrm{Na}_{2} \mathrm{O}-\mathrm{Co}(211)-1 \times 3$

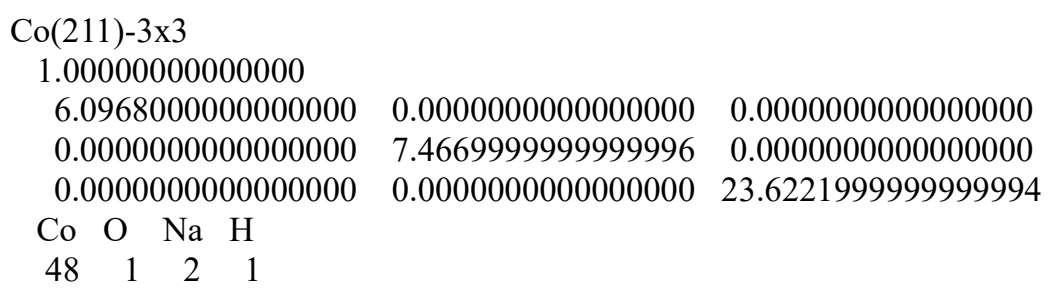

Selective dynamics

Direct

$\begin{array}{llllllll}0.0039768403096687 & 0.1666663988214765 & 0.9745822997011331 & F & F & F\end{array}$ $\begin{array}{lllllllll}0.0039765122687285 & 0.5000000000000000 & 0.9745822997011331 & F & F & F\end{array}$ $\begin{array}{llllllll}0.0039768403096687 & 0.83333336011785235 & 0.9745822997011331 & F & F & F\end{array}$ 
$\begin{array}{llllllll}0.3315888662905095 & 0.0000000000000000 & 0.0034278771663878 & F & F & F\end{array}$ $\begin{array}{llllllll}0.3315855858811219 & 0.3333350743270387 & 0.0034291471581795 & F & F & F\end{array}$ $\begin{array}{lllllllll}0.3315855858811219 & 0.6666647917503710 & 0.0034291471581795 & F & F & F\end{array}$ $\begin{array}{lllllllll}0.6664853693741009 & 0.1666655952859273 & 0.0285661792720404 & F & F & F\end{array}$ $\begin{array}{llllllll}0.6664884857630327 & 0.5000000000000000 & 0.0285654596100287 & F & F & F\end{array}$ $\begin{array}{lllllllll}0.6664853693741009 & 0.8333344047140727 & 0.0285661792720404 & F & F & F\end{array}$ $\begin{array}{llllllll}0.9983825941477491 & 0.0000000000000000 & 0.0609119811025209 & F & F & F\end{array}$ $\begin{array}{llllllll}0.9983791497178913 & 0.33333453863666702 & 0.0609079171288016 & F & F & F\end{array}$ $\begin{array}{llllllll}0.9983791497178913 & 0.6666654613633298 & 0.0609079171288016 & F & F & F\end{array}$ $\begin{array}{lllllllll}0.3314264860254568 & 0.1666650595955517 & 0.0918800111759310 & F & F & F\end{array}$ $\begin{array}{llllllll}0.3314281262301506 & 0.5000000000000000 & 0.0918805615056968 & F & F & F\end{array}$ $\begin{array}{llllllll}0.3314264860254568 & 0.83333449404044483 & 0.0918800111759310 & \mathrm{~F} & \mathrm{~F} & \mathrm{~F}\end{array}$ $\begin{array}{llllllll}0.6654751673008832 & 0.0000000000000000 & 0.1205095207050988 & F & F & F\end{array}$ $\begin{array}{llllllll}0.6654631938065876 & 0.3333330654881479 & 0.1205040597404121 & F & F & F\end{array}$ $\begin{array}{llllllll}0.6654631938065876 & 0.6666669345118521 & 0.1205040597404121 & F & F & F\end{array}$ $\begin{array}{llllllll}0.0009121178323070 & 0.1666669345118521 & 0.1526111031148716 & F & F & F\end{array}$ $\begin{array}{llllllll}0.0009114617504267 & 0.5000000000000000 & 0.1526112301140472 & F & F & F\end{array}$ $\begin{array}{llllllll}0.0009121178323070 & 0.8333330654881479 & 0.1526111031148716 & F & F & F\end{array}$ $\begin{array}{llllllll}0.3340214538774404 & 0.0000000000000000 & 0.1833969740329024 & F & F & F\end{array}$ $\begin{array}{lllllll}0.3340229300616713 & 0.3333327976429601 & 0.1833968470337197 & F & F & F\end{array}$ $\begin{array}{llllllll}0.3340229300616713 & 0.6666672023570399 & 0.1833968470337197 & F & F & F\end{array}$ $\begin{array}{llllllll}0.6659674072033428 & 0.1664263389120203 & 0.2117668895214391 & \mathrm{~T} & \mathrm{~T} & \mathrm{~T}\end{array}$ $\begin{array}{llllllll}0.6667693936345407 & 0.5002760327314161 & 0.2118509531235440 & \mathrm{~T} & \mathrm{~T} & \mathrm{~T}\end{array}$ $\begin{array}{llllllll}0.6653433747384949 & 0.8334116784821781 & 0.2118960691086768 & \mathrm{~T} & \mathrm{~T} & \mathrm{~T}\end{array}$ $\begin{array}{lllllllll}0.9973997909551519 & -0.0003122570896731 & 0.2433806437166484 & \text { T } & \text { T } & \text { T }\end{array}$ $\begin{array}{llllllll}0.9995481227378127 & 0.3336027187832307 & 0.2429975203508628 & \mathrm{~T} & \mathrm{~T} & \mathrm{~T}\end{array}$ $\begin{array}{llllllll}0.9985525395186134 & 0.6666895449455533 & 0.2432959106060703 & \mathrm{~T} & \mathrm{~T} & \mathrm{~T}\end{array}$ $\begin{array}{llllllll}0.3328580974270715 & 0.1655785705700051 & 0.2746161923291268 & \mathrm{~T} & \mathrm{~T} & \mathrm{~T}\end{array}$ $\begin{array}{llllllll}0.3332241377078415 & 0.5002930771315897 & 0.2742088353183476 & \mathrm{~T} & \mathrm{~T} & \mathrm{~T}\end{array}$ $\begin{array}{lllllllll}0.3315131961132983 & 0.8347246966246131 & 0.2751004349187668 & \mathrm{~T} & \mathrm{~T} & \mathrm{~T}\end{array}$ $\begin{array}{llllllll}0.6653396770328229 & -0.0008081309944815 & 0.3033598937086599 & \text { T } & \text { T } & \text { T }\end{array}$ $\begin{array}{llllllllll}0.6679843797271969 & 0.3331835352745552 & 0.3023765102583033 & \mathrm{~T} & \mathrm{~T} & \mathrm{~T}\end{array}$ $\begin{array}{llllllllll}0.6659635308600181 & 0.6680648032882724 & 0.3031725694016482 & \mathrm{~T} & \mathrm{~T} & \mathrm{~T}\end{array}$ $\begin{array}{llllllll}0.0000400588793651 & 0.1670658812229724 & 0.3342563432456641 & \mathrm{~T} & \mathrm{~T} & \mathrm{~T}\end{array}$ $\begin{array}{llllllllll}0.9994525148598531 & 0.5001366688914094 & 0.3353904551737359 & \mathrm{~T} & \mathrm{~T} & \mathrm{~T}\end{array}$ $\begin{array}{llllllll}-0.0018583022633239 & 0.8327100540194835 & 0.3354567905052871 & \mathrm{~T} & \mathrm{~T} & \mathrm{~T}\end{array}$ $\begin{array}{lllllll}0.3295105437939897 & 0.0006240829150966 & 0.3687839288582370 & \mathrm{~T} & \mathrm{~T} & \mathrm{~T}\end{array}$ $\begin{array}{lllllllllllll}0.3305647794254619 & 0.3319350656628934 & 0.3631585073860314 & \mathrm{~T} & \mathrm{~T}\end{array}$ $\begin{array}{llllllllll}0.3309203296335538 & 0.6681737108801794 & 0.3633547672211947 & \mathrm{~T} & \mathrm{~T} & \mathrm{~T}\end{array}$ $\begin{array}{llllllll}0.6676872512229978 & 0.1677214065306828 & 0.3906954619114150 & \mathrm{~T} & \mathrm{~T} & \mathrm{~T}\end{array}$ $\begin{array}{llllllllll}0.6621621028227725 & 0.4991538806348371 & 0.3899526772605293 & \mathrm{~T} & \mathrm{~T} & \mathrm{~T}\end{array}$ $\begin{array}{llllllll}0.6657119782042966 & 0.8354398748009635 & 0.3936715376351596 & \mathrm{~T} & \mathrm{~T} & \mathrm{~T}\end{array}$ $\begin{array}{lllllll}0.9898024429791771 & 0.0037537819084640 & 0.4214428578565002 & \mathrm{~T} & \mathrm{~T} & \mathrm{~T}\end{array}$ $\begin{array}{llllllll}0.9918042048101675 & 0.3348655085854445 & 0.4246363479335738 & \mathrm{~T} & \mathrm{~T} & \mathrm{~T}\end{array}$ $\begin{array}{llllllll}0.9798836401425487 & 0.6597002625489501 & 0.4272063463157648 & \mathrm{~T} & \mathrm{~T} & \mathrm{~T}\end{array}$ $\begin{array}{llllllll}0.1077746489631742 & 0.4930348805290349 & 0.4773843298196017 & \mathrm{~T} & \mathrm{~T} & \mathrm{~T}\end{array}$ $\begin{array}{llllllllll}0.3731510549304654 & 0.7261266000961475 & 0.4845517654860206 & \mathrm{~T} & \mathrm{~T} & \mathrm{~T}\end{array}$ $\begin{array}{lllllllll}0.3824550594976283 & 0.2686625998102146 & 0.4844913970343471 & \mathrm{~T} & \mathrm{~T} & \mathrm{~T}\end{array}$ $\begin{array}{llllllllll}0.8337157486039309 & 0.8396497384614255 & 0.4545634632904084 & \mathrm{~T} & \mathrm{~T} & \mathrm{~T}\end{array}$

18) $\mathrm{CO}-\mathrm{Co}(211)-1 \mathrm{x} 3$

$\operatorname{Co}(211)-3 \times 3$

1.00000000000000 


$$
\begin{array}{ccc}
6.0968000000000000 & 0.0000000000000000 & 0.0000000000000000 \\
0.0000000000000000 & 7.4669999999999996 & 0.0000000000000000 \\
0.0000000000000000 & 0.0000000000000000 & 23.62219999999999994
\end{array}
$$

$\mathrm{Co} \mathrm{C} \mathrm{O}$

$\begin{array}{lll}48 & 1 & 1\end{array}$

Selective dynamics

Direct

$\begin{array}{llllllll}0.0039768403096687 & 0.1666663988214836 & 0.9745822997011260 & F & F & F\end{array}$ $\begin{array}{lllllllll}0.0039765122687285 & 0.5000000000000000 & 0.9745819610366553 & F & F & F\end{array}$ $\begin{array}{lllllllll}0.0039768403096687 & 0.8333336011785164 & 0.9745822997011260 & F & F & F\end{array}$ $\begin{array}{lllllllll}0.3315888662905095 & 0.0000000000000000 & 0.0034278771663949 & F & F & F\end{array}$ $\begin{array}{llllllll}0.3315855858811148 & 0.3333350743270387 & 0.0034291471581795 & F & F & F\end{array}$ $\begin{array}{llllllll}0.3315855858811148 & 0.6666647917503710 & 0.0034291471581795 & F & F & F\end{array}$ $\begin{array}{llllllll}0.6664853693741009 & 0.1666655952859273 & 0.0285661792720404 & F & F & F\end{array}$ $\begin{array}{lllllllll}0.6664884857630256 & 0.5000000000000000 & 0.0285654596100287 & F & F & F\end{array}$ $\begin{array}{llllllll}0.6664853693741009 & 0.8333344047140727 & 0.0285661792720404 & F & F & F\end{array}$ $\begin{array}{lllllllll}0.9983825941477491 & 0.0000000000000000 & 0.0609119811025209 & F & F & F\end{array}$ $\begin{array}{llllllll}0.9983791497178842 & 0.3333345386366702 & 0.0609079171288016 & F & F & F\end{array}$ $\begin{array}{llllllll}0.9983791497178842 & 0.6666654613633298 & 0.0609079171288016 & F & F & F\end{array}$ $\begin{array}{lllllllll}0.3314264860254568 & 0.1666650595955517 & 0.0918800111759310 & F & F & F\end{array}$ $\begin{array}{lllllllll}0.3314281262301506 & 0.5000000000000000 & 0.0918805615057039 & F & F & F\end{array}$ $\begin{array}{llllllll}0.3314264860254568 & 0.8333349404044483 & 0.0918800111759310 & F & F & F\end{array}$ $\begin{array}{llllllllll}0.6654751673008761 & 0.0000000000000000 & 0.1205095207050988 & F & F & F\end{array}$ $\begin{array}{lllllllll}0.6654631938065876 & 0.3333330654881479 & 0.1205040597404121 & F & F & F\end{array}$ $\begin{array}{llllllll}0.6654631938065876 & 0.6666669345118521 & 0.1205040597404121 & F & F & F\end{array}$ $\begin{array}{llllllll}0.0009121178323070 & 0.1666669345118521 & 0.1526111031148645 & F & F & F\end{array}$ $\begin{array}{llllllll}0.0009114617504267 & 0.5000000000000000 & 0.1526112301140472 & F & F & F\end{array}$ $\begin{array}{llllllll}0.0009121178323070 & 0.8333330654881479 & 0.1526111031148645 & F & F & F\end{array}$ $\begin{array}{llllllllll}0.3340214538774404 & 0.0000000000000000 & 0.1833969740329024 & F & F & F\end{array}$ $\begin{array}{lllllllll}0.3340229300616713 & 0.3333327976429601 & 0.1833968470337197 & F & F & F\end{array}$ $\begin{array}{lllllllll}0.3340229300616713 & 0.6666672023570399 & 0.1833968470337197 & F & F & F\end{array}$ $\begin{array}{llllllll}0.6661549991465248 & 0.1671088045642204 & 0.2121370482318834 & \mathrm{~T} & \mathrm{~T} & \mathrm{~T}\end{array}$ $\begin{array}{llllllll}0.6652657502845153 & 0.5000015103498405 & 0.2121330654994987 & \mathrm{~T} & \mathrm{~T} & \mathrm{~T}\end{array}$ $\begin{array}{lllllll}0.6661124607590213 & 0.8328841079110644 & 0.2121329283428572 & \mathrm{~T} & \mathrm{~T} & \mathrm{~T}\end{array}$ $\begin{array}{lllllll}0.9994160749341340 & 0.0000052536044698 & 0.2426842257032323 & \mathrm{~T} & \mathrm{~T} & \mathrm{~T}\end{array}$ $\begin{array}{lllllll}0.9985746210866836 & 0.3340245771292583 & 0.2426360101780421 & \mathrm{~T} & \mathrm{~T} & \mathrm{~T}\end{array}$ $\begin{array}{llllllll}0.9985342947834118 & 0.6659424189576791 & 0.2426283974096319 & \mathrm{~T} & \mathrm{~T} & \mathrm{~T}\end{array}$ $\begin{array}{lllllll}0.3343453746893962 & 0.1669266906403577 & 0.2746075407314732 & \mathrm{~T} & \mathrm{~T} & \mathrm{~T}\end{array}$ $\begin{array}{lllllll}0.3333483841401463 & 0.4999846212555771 & 0.2753018031418745 & \mathrm{~T} & \mathrm{~T} & \mathrm{~T}\end{array}$ $\begin{array}{lllllllll}0.3343338649606651 & 0.8330155988231027 & 0.2746101084265001 & \mathrm{~T} & \mathrm{~T} & \mathrm{~T}\end{array}$ $\begin{array}{lllllll}0.6682658596518518 & -0.0000269536024985 & 0.3032248522666768 & \mathrm{~T} & \mathrm{~T} & \mathrm{~T}\end{array}$ $\begin{array}{llllllll}0.6671068968686519 & 0.3335707328329204 & 0.3038066872712201 & \mathrm{~T} & \mathrm{~T} & \mathrm{~T}\end{array}$ $\begin{array}{lllllllll}0.6671364186720850 & 0.6663448520771074 & 0.3038149424995706 & \mathrm{~T} & \mathrm{~T} & \mathrm{~T}\end{array}$ $\begin{array}{llllllll}0.0018695158547401 & 0.1659493767865665 & 0.3331735140198635 & \mathrm{~T} & \mathrm{~T} & \mathrm{~T}\end{array}$ $\begin{array}{lllllll}-0.0008970692702314 & 0.4999835214599412 & 0.3352222951670533 & \mathrm{~T} & \mathrm{~T} & \mathrm{~T}\end{array}$ $\begin{array}{lllllll}0.0019135053750565 & 0.8340723162933716 & 0.3331646005911059 & \mathrm{~T} & \mathrm{~T} & \mathrm{~T}\end{array}$ $\begin{array}{llllllll}0.3324077884408947 & -0.0000152370560420 & 0.3661302008868556 & \mathrm{~T} & \mathrm{~T} & \mathrm{~T}\end{array}$ $\begin{array}{llllllll}0.3344893868202183 & 0.3334477335242045 & 0.3662386227600017 & \mathrm{~T} & \mathrm{~T} & \mathrm{~T}\end{array}$ $\begin{array}{llllllll}0.3344707664866270 & 0.6664445859190905 & 0.3662489140089750 & \mathrm{~T} & \mathrm{~T} & \mathrm{~T}\end{array}$ $\begin{array}{lllllllll}0.6680993464519451 & 0.1656058362065947 & 0.3913286873459751 & \mathrm{~T} & \mathrm{~T} & \mathrm{~T}\end{array}$ $\begin{array}{llllllll}0.6659113234414806 & 0.4999704824390104 & 0.3951486098969861 & \mathrm{~T} & \mathrm{~T} & \mathrm{~T}\end{array}$ $\begin{array}{llllllll}0.6681063797376795 & 0.8343574477438920 & 0.3913235367892759 & \mathrm{~T} & \mathrm{~T} & \mathrm{~T}\end{array}$ $\begin{array}{llllll}0.9977770183830449 & -0.0000074470223833 & 0.4189927122397781 & T & T & T\end{array}$ $\begin{array}{lllllll}-0.0060628242549301 & 0.3290503972765261 & 0.4219669693703065 & \mathrm{~T} & \mathrm{~T} & \mathrm{~T}\end{array}$ $\begin{array}{lllllll}-0.0061290600394377 & 0.6709379384791161 & 0.4219736221732861 & T & T & T\end{array}$ 
$\begin{array}{lllllll}0.8437213107813394 & 0.4999488573397495 & 0.4696933805210059 & \mathrm{~T} & \mathrm{~T} & \mathrm{~T}\end{array}$ $\begin{array}{llllllll}0.7858516594498068 & 0.4998363955114090 & 0.5181969527513248 & \mathrm{~T} & \mathrm{~T} & \mathrm{~T}\end{array}$

19) $\mathrm{CO}-\mathrm{PrO}_{2}-\mathrm{Co}(211)-1 \times 3$

$\begin{array}{rrr}\operatorname{Co}(211)-3 \times 3 & & \\ 1.00000000000000 & & \\ 6.0968000000000000 & 0.0000000000000000 & 0.0000000000000000 \\ 0.0000000000000000 & 7.4669999999999996 & 0.0000000000000000 \\ 0.0000000000000000 & 0.0000000000000000 & 23.6221999999999994\end{array}$

$\mathrm{Co} \operatorname{Pr} \mathrm{O} \mathrm{C}$

$\begin{array}{llll}48 & 1 & 3 & 1\end{array}$

Selective dynamics

Direct

$\begin{array}{llllllll}0.0039768403096687 & 0.1666663988214765 & 0.9745822997011331 & F & F & F\end{array}$ $\begin{array}{lllllllllll}0.0039765122687285 & 0.5000000000000000 & 0.9745822997011331 & F & F & F\end{array}$ $\begin{array}{llllllll}0.0039768403096687 & 0.8333336011785235 & 0.9745822997011331 & F & F & F\end{array}$ $\begin{array}{lllllllll}0.3315888662905095 & 0.0000000000000000 & 0.0034278771663878 & F & F & F\end{array}$ $\begin{array}{llllllll}0.3315855858811219 & 0.3333350743270387 & 0.0034291471581795 & F & F & F\end{array}$ $\begin{array}{llllllll}0.3315855858811219 & 0.6666647917503710 & 0.0034291471581795 & F & F & F\end{array}$ $\begin{array}{llllllll}0.6664853693741009 & 0.1666655952859273 & 0.0285661792720404 & F & F & F\end{array}$ $\begin{array}{lllllllll}0.6664884857630327 & 0.5000000000000000 & 0.0285654596100287 & F & F & F\end{array}$ $\begin{array}{lllllllll}0.6664853693741009 & 0.8333344047140727 & 0.0285661792720404 & F & F & F\end{array}$ $\begin{array}{llllllll}0.9983825941477491 & 0.0000000000000000 & 0.0609119811025209 & F & F & F\end{array}$ $\begin{array}{llllllll}0.9983791497178913 & 0.3333345386366702 & 0.0609079171288016 & F & F & F\end{array}$ $\begin{array}{lllllllll}0.9983791497178913 & 0.6666654613633298 & 0.0609079171288016 & F & F & F\end{array}$ $\begin{array}{lllllllll}0.3314264860254568 & 0.1666650595955517 & 0.0918800111759310 & F & F & F\end{array}$ $\begin{array}{lllllllll}0.3314281262301506 & 0.5000000000000000 & 0.0918805615056968 & F & F & F\end{array}$ $\begin{array}{llllllll}0.3314264860254568 & 0.8333349404044483 & 0.0918800111759310 & F & F & F\end{array}$ $\begin{array}{llllllllll}0.6654751673008832 & 0.0000000000000000 & 0.1205095207050988 & F & F & F\end{array}$ $\begin{array}{llllllll}0.6654631938065876 & 0.3333330654881479 & 0.1205040597404121 & F & F & F\end{array}$ $\begin{array}{llllllll}0.6654631938065876 & 0.6666669345118521 & 0.1205040597404121 & F & F & F\end{array}$ $\begin{array}{lllllll}0.0009121178323070 & 0.1666669345118521 & 0.1526111031148716 & F & F & F\end{array}$ $\begin{array}{llllllll}0.0009114617504267 & 0.5000000000000000 & 0.1526112301140472 & F & F & F\end{array}$ $\begin{array}{llllllll}0.0009121178323070 & 0.8333330654881479 & 0.1526111031148716 & F & F & F\end{array}$ $\begin{array}{llllllll}0.3340214538774404 & 0.0000000000000000 & 0.1833969740329024 & F & F & F\end{array}$ $\begin{array}{llllllll}0.3340229300616713 & 0.3333327976429601 & 0.1833968470337197 & F & F & F\end{array}$ $\begin{array}{llllllll}0.3340229300616713 & 0.6666672023570399 & 0.1833968470337197 & F & F & F\end{array}$ $\begin{array}{llllllll}0.6653613728124529 & 0.1664578260424591 & 0.2117980719468279 & \mathrm{~T} & \mathrm{~T} & \mathrm{~T}\end{array}$ $\begin{array}{llllllll}0.6666586309066196 & 0.5000313797493514 & 0.2119408757669775 & \mathrm{~T} & \mathrm{~T} & \mathrm{~T}\end{array}$ $\begin{array}{lllllll}0.6659914870899454 & 0.8341629071782054 & 0.2119018324888515 & \mathrm{~T} & \mathrm{~T} & \mathrm{~T}\end{array}$ $\begin{array}{lllllll}0.9974934696656351 & 0.0004976302811139 & 0.2433895034643271 & \mathrm{~T} & \mathrm{~T} & \mathrm{~T}\end{array}$ $\begin{array}{lllllll}-0.0002633123707830 & 0.3337693069221044 & 0.2433310962050885 & \mathrm{~T} & \mathrm{~T} & \mathrm{~T}\end{array}$ $\begin{array}{lllllll}0.0005911106266107 & 0.6668874065175531 & 0.2430092377945242 & \mathrm{~T} & \mathrm{~T} & \mathrm{~T}\end{array}$ $\begin{array}{lllllll}0.3323331229694900 & 0.1673259507555524 & 0.2755509830768902 & \mathrm{~T} & \mathrm{~T} & \mathrm{~T}\end{array}$ $\begin{array}{lllllll}0.3355354171547904 & 0.4991583807736408 & 0.2752810473085250 & \mathrm{~T} & \mathrm{~T} & \mathrm{~T}\end{array}$ $\begin{array}{llllllll}0.3323926484615387 & 0.8349771752551072 & 0.2742738062374915 & \mathrm{~T} & \mathrm{~T} & \mathrm{~T}\end{array}$ $\begin{array}{llllllll}0.6656360126150809 & 0.0020555510077673 & 0.3030445174250208 & \mathrm{~T} & \mathrm{~T} & \mathrm{~T}\end{array}$ $\begin{array}{lllllll}0.6683208220882650 & 0.3299632273938333 & 0.3022326110535698 & \mathrm{~T} & \mathrm{~T} & \mathrm{~T}\end{array}$ $\begin{array}{llllllll}0.6661856088351116 & 0.6675800086966346 & 0.3017751356461609 & \mathrm{~T} & \mathrm{~T} & \mathrm{~T}\end{array}$ $\begin{array}{lllllll}-0.0002903970610149 & 0.1689617938794409 & 0.3346053250353859 & \mathrm{~T} & \mathrm{~T} & \mathrm{~T}\end{array}$ $\begin{array}{lllllllll}0.0039700054823608 & 0.4995107179755596 & 0.3342867432952809 & \mathrm{~T} & \mathrm{~T} & \mathrm{~T}\end{array}$ $\begin{array}{llllllll}0.9970602132120677 & 0.8336056345382030 & 0.3356768295778463 & \mathrm{~T} & \mathrm{~T} & \mathrm{~T}\end{array}$ $\begin{array}{llllllll}0.3282651795382773 & 0.9974178354674614 & 0.3656643359298637 & T & T & T\end{array}$ 
$\begin{array}{lllllll}0.3273469268939008 & 0.3330144894485049 & 0.3691608229220192 & \mathrm{~T} & \mathrm{~T} & \mathrm{~T}\end{array}$ $\begin{array}{llllllllll}0.3279534198033027 & 0.6753140657828917 & 0.3640657939966930 & \mathrm{~T} & \mathrm{~T} & \mathrm{~T}\end{array}$ $\begin{array}{lllllllll}0.6703289490984979 & 0.1641818773280594 & 0.3950580129608196 & \mathrm{~T} & \mathrm{~T} & \mathrm{~T}\end{array}$ $\begin{array}{lllllllll}0.6719650682442321 & 0.4998072543593880 & 0.3867644858655807 & \mathrm{~T} & \mathrm{~T} & \mathrm{~T}\end{array}$ $\begin{array}{lllllllll}0.6597528975219376 & 0.8344673572543855 & 0.3923723650380088 & \mathrm{~T} & \mathrm{~T} & \mathrm{~T}\end{array}$ $\begin{array}{lllllll}0.9859509994381287 & -0.0002994464043558 & 0.4228900076596769 & \text { T } & \text { T } & \text { T }\end{array}$ $\begin{array}{llllllll}0.0029436661185110 & 0.3351652724672987 & 0.4258953541330976 & \mathrm{~T} & \mathrm{~T} & \mathrm{~T}\end{array}$ $\begin{array}{lllllllllll}0.9797222719183487 & 0.6663610553999267 & 0.4266669529553186 & \mathrm{~T} & \mathrm{~T} & \mathrm{~T}\end{array}$ $\begin{array}{llllllllll}0.4173386133427402 & 0.5935132845643734 & 0.4855392530519371 & \mathrm{~T} & \mathrm{~T} & \mathrm{~T}\end{array}$ $\begin{array}{llllllllll}0.5193082221195895 & 0.3417149760160189 & 0.4403055226885715 & \mathrm{~T} & \mathrm{~T} & \mathrm{~T}\end{array}$ $\begin{array}{llllllllll}0.0644925684256396 & 0.4991640065587024 & 0.4839528006760478 & \mathrm{~T} & \mathrm{~T} & \mathrm{~T}\end{array}$ $\begin{array}{llllllll}0.7067156391182440 & 0.8277240629360844 & 0.5130051754416428 & \mathrm{~T} & \mathrm{~T} & \mathrm{~T}\end{array}$ $\begin{array}{llllllllll}0.7988236910497837 & 0.8415803443599996 & 0.4655792983481067 & \mathrm{~T} & \mathrm{~T} & \mathrm{~T}\end{array}$

\section{0) $\mathrm{CO}-\mathrm{Na}_{2} \mathrm{O}-\mathrm{Co}(211)-1 \times 3$}

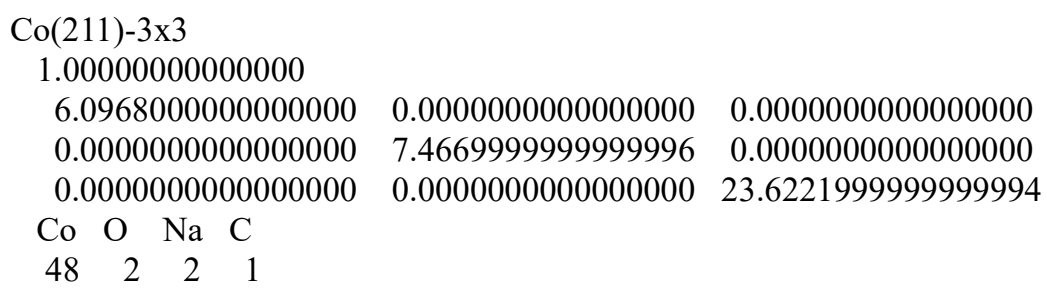

Selective dynamics

Direct

$\begin{array}{lllllllll}0.0039768403096687 & 0.1666663988214765 & 0.9745822997011331 & F & F & F\end{array}$ $\begin{array}{lllllllll}0.0039765122687285 & 0.5000000000000000 & 0.9745822997011331 & F & F & F\end{array}$ $\begin{array}{lllllllll}0.0039768403096687 & 0.8333336011785235 & 0.9745822997011331 & F & F & F\end{array}$ $\begin{array}{llllllll}0.3315888662905095 & 0.0000000000000000 & 0.0034278771663878 & F & F & F\end{array}$ $\begin{array}{llllllll}0.3315855858811219 & 0.3333350743270387 & 0.0034291471581795 & \mathrm{~F} & \mathrm{~F} & \mathrm{~F}\end{array}$ $\begin{array}{llllllll}0.3315855858811219 & 0.6666647917503710 & 0.0034291471581795 & F & F & F\end{array}$ $\begin{array}{llllllll}0.6664853693741009 & 0.1666655952859273 & 0.0285661792720404 & F & F & F\end{array}$ $\begin{array}{llllllll}0.6664884857630327 & 0.5000000000000000 & 0.0285654596100287 & F & F & F\end{array}$ $\begin{array}{lllllllll}0.6664853693741009 & 0.8333344047140727 & 0.0285661792720404 & F & F & F\end{array}$ $\begin{array}{lllllllll}0.9983825941477491 & 0.0000000000000000 & 0.0609119811025209 & F & F & F\end{array}$ $\begin{array}{llllllll}0.9983791497178913 & 0.3333345386366702 & 0.0609079171288016 & F & F & F\end{array}$ $\begin{array}{llllllll}0.9983791497178913 & 0.6666654613633298 & 0.0609079171288016 & F & F & F\end{array}$ $\begin{array}{llllllll}0.3314264860254568 & 0.1666650595955517 & 0.0918800111759310 & F & F & F\end{array}$ $\begin{array}{llllllll}0.3314281262301506 & 0.5000000000000000 & 0.0918805615056968 & F & F & F\end{array}$ $\begin{array}{llllllll}0.3314264860254568 & 0.8333349404044483 & 0.0918800111759310 & F & F & F\end{array}$ $\begin{array}{llllllll}0.6654751673008832 & 0.0000000000000000 & 0.1205095207050988 & F & F & F\end{array}$ $\begin{array}{lllllllll}0.6654631938065876 & 0.3333330654881479 & 0.1205040597404121 & F & F & F\end{array}$ $\begin{array}{lllllllll}0.6654631938065876 & 0.6666669345118521 & 0.1205040597404121 & F & F & F\end{array}$ $\begin{array}{lllllll}0.0009121178323070 & 0.1666669345118521 & 0.1526111031148716 & F & F & F\end{array}$ $\begin{array}{llllllll}0.0009114617504267 & 0.5000000000000000 & 0.1526112301140472 & F & F & F\end{array}$ $\begin{array}{lllllll}0.0009121178323070 & 0.8333330654881479 & 0.1526111031148716 & F & F & F\end{array}$ $\begin{array}{llllllllll}0.3340214538774404 & 0.0000000000000000 & 0.1833969740329024 & F & F & F\end{array}$ $\begin{array}{llllllll}0.3340229300616713 & 0.3333327976429601 & 0.1833968470337197 & F & F & F\end{array}$ $\begin{array}{llllllll}0.3340229300616713 & 0.6666672023570399 & 0.1833968470337197 & F & F & F\end{array}$ $\begin{array}{llllllllll}0.6658645422707269 & 0.1660374172918767 & 0.2117900980960461 & \mathrm{~T} & \mathrm{~T} & \mathrm{~T}\end{array}$ $\begin{array}{lllllll}0.6667366244340689 & 0.5004382308647629 & 0.2118554643162825 & \mathrm{~T} & \mathrm{~T} & \mathrm{~T}\end{array}$ $\begin{array}{lllllllllll}0.6648505018371085 & 0.8330335876520153 & 0.2119251929010326 & \mathrm{~T} & \mathrm{~T} & \mathrm{~T}\end{array}$ $\begin{array}{lllllllll}0.9976500271947548 & -0.0009152142789934 & 0.2434567089120533 & \text { T } & \text { T } & \text { T }\end{array}$ $\begin{array}{lllllllll}0.9994419541752373 & 0.3336727498136443 & 0.2433463717582243 & \mathrm{~T} & \mathrm{~T} & \mathrm{~T}\end{array}$ 
$\begin{array}{lllllll}0.9986802249148526 & 0.6672305147521883 & 0.2433768180795012 & \mathrm{~T} & \mathrm{~T} & \mathrm{~T}\end{array}$ $\begin{array}{llllllll}0.3325205505817793 & 0.1656167447402343 & 0.2744783234707141 & \mathrm{~T} & \mathrm{~T} & \mathrm{~T}\end{array}$ $\begin{array}{llllllllll}0.3323202207837498 & 0.5005203464497577 & 0.2741292923054650 & \mathrm{~T} & \mathrm{~T} & \mathrm{~T}\end{array}$ $\begin{array}{llllllllll}0.3313032653954628 & 0.8344843688483434 & 0.2754030764850086 & \mathrm{~T} & \mathrm{~T} & \mathrm{~T}\end{array}$ $\begin{array}{llllllll}0.6653438035873240 & -0.0001451086055465 & 0.3030014726379174 & \mathrm{~T} & \mathrm{~T} & \mathrm{~T}\end{array}$ $\begin{array}{llllllllll}0.6664557620174970 & 0.3336835320680231 & 0.3023131973640921 & \mathrm{~T} & \mathrm{~T} & \mathrm{~T}\end{array}$ $\begin{array}{llllllllll}0.6646856522760883 & 0.6668688022120725 & 0.3030835601043886 & \text { T } & \text { T } & \text { T }\end{array}$ $\begin{array}{lllllllll}-0.0003733117933651 & 0.1676819447939709 & 0.3335882412674660 & \text { T } & \text { T } & \text { T }\end{array}$ $\begin{array}{llllllllll}0.9983961010769162 & 0.5012002018953704 & 0.3349992166482182 & \mathrm{~T} & \mathrm{~T} & \mathrm{~T}\end{array}$ $\begin{array}{llllllllll}-0.0028299828258668 & 0.8334750718135748 & 0.3365147381389103 & \mathrm{~T} & \mathrm{~T} & \mathrm{~T}\end{array}$ $\begin{array}{llllllllll}0.3290327276351646 & 0.0015081270937850 & 0.3681854646085312 & \mathrm{~T} & \mathrm{~T} & \mathrm{~T}\end{array}$ $\begin{array}{lllllllll}0.3303835492779598 & 0.3325073003050363 & 0.3632174238355844 & \mathrm{~T} & \mathrm{~T} & \mathrm{~T}\end{array}$ $\begin{array}{llllllll}0.3302292419764821 & 0.6686320756678014 & 0.3637227706046005 & \mathrm{~T} & \mathrm{~T} & \mathrm{~T}\end{array}$ $\begin{array}{lllllllllll}0.6673245832511625 & 0.1685238972889263 & 0.3898079691366706 & \mathrm{~T} & \mathrm{~T} & \mathrm{~T}\end{array}$ $\begin{array}{llllllllll}0.6630032573437643 & 0.4975559461748061 & 0.3897105084566692 & \mathrm{~T} & \mathrm{~T} & \mathrm{~T}\end{array}$ $\begin{array}{llllllllll}0.6607745510081795 & 0.8346430522056604 & 0.3933386399598129 & \mathrm{~T} & \mathrm{~T} & \mathrm{~T}\end{array}$ $\begin{array}{llllllll}0.9834259385443012 & 0.0038732714809830 & 0.4230745867549848 & \mathrm{~T} & \mathrm{~T} & \mathrm{~T}\end{array}$ $\begin{array}{lllllllll}0.9955426878540420 & 0.3325118661102236 & 0.4238595857025066 & \mathrm{~T} & \mathrm{~T} & \mathrm{~T}\end{array}$ $\begin{array}{llllllll}0.9780984236133625 & 0.6590038108232589 & 0.4304189966252635 & \mathrm{~T} & \mathrm{~T} & \mathrm{~T}\end{array}$ $\begin{array}{llllllll}0.1171919195741866 & 0.4895854190147851 & 0.4757554388491282 & \text { T } & \text { T } & \text { T }\end{array}$ $\begin{array}{llllllll}0.7185608969788112 & 0.8439948225265722 & 0.5142339662466496 & \mathrm{~T} & \mathrm{~T} & \mathrm{~T}\end{array}$ $\begin{array}{llllllllll}0.3681024528313128 & 0.7316150541463173 & 0.4908880157680375 & \mathrm{~T} & \mathrm{~T} & \mathrm{~T}\end{array}$ $\begin{array}{lllllllllll}0.3889793510931684 & 0.2640342996130064 & 0.4860592201200274 & \mathrm{~T} & \mathrm{~T} & \mathrm{~T}\end{array}$ $\begin{array}{llllllllllll}0.8139449779826161 & 0.8430928781916557 & 0.4676575895445056 & \mathrm{~T} & \mathrm{~T} & \mathrm{~T}\end{array}$

21) $\mathrm{C}_{2} \mathrm{H}_{4}-\mathrm{Co}(111)-3 \times 3$

Co C H

1.00000000000000

$\begin{array}{llll}7.4587998390197754 & 0.0000000000000000 & 0.0000000000000000\end{array}$

$\begin{array}{llll}-3.7294002959821051 & 6.4595099249780574 & 0.0000000000000000\end{array}$

$\begin{array}{lll}-0.0000010106117090 & -0.0000017504309440 & 23.1201000213622159\end{array}$

Co C H

$\begin{array}{lll}36 & 2 & 4\end{array}$

Selective dynamics

Direct

$\begin{array}{llllllll}0.0000352770987675 & 0.9999648825964869 & 0.0907110695049838 & F & F & F\end{array}$ $\begin{array}{llllllll}0.3333557427970177 & 0.0000001793936022 & 0.0907175141137841 & F & F & F\end{array}$ $\begin{array}{llllllll}0.6666918534736581 & 0.9999938321653516 & 0.0907267268766958 & F & F & F\end{array}$ $\begin{array}{lllllllll}0.0000062578975388 & 0.3333084372988537 & 0.0907267268766958 & \mathrm{~F} & \mathrm{~F} & \mathrm{~F}\end{array}$ $\begin{array}{lllllllll}0.3333357007148621 & 0.3333426504206471 & 0.0907265971194704 & F & F & F\end{array}$ $\begin{array}{llllllllll}0.6666945969032270 & 0.3333051862794676 & 0.0907313116319486 & F & F & F\end{array}$ $\begin{array}{llllllll}0.9999998575876035 & 0.6666444062796870 & 0.0907175141137841 & F & F & F\end{array}$ $\begin{array}{llllllll}0.3333385397113773 & 0.6666614354387903 & 0.0907302303217463 & F & F & F\end{array}$ $\begin{array}{lllllllll}0.6666573639132167 & 0.6666642220269168 & 0.0907265971194704 & F & F & F\end{array}$ $\begin{array}{llllllll}0.1111175321760314 & 0.2222028177229376 & 0.1750651163386081 & F & F & F\end{array}$ $\begin{array}{llllllll}0.4444257597051688 & 0.2222195372577005 & 0.1750655488626904 & \mathrm{~F} & \mathrm{~F} & \mathrm{~F}\end{array}$ $\begin{array}{llllllll}0.7777781372906531 & 0.2222218594174734 & 0.1750735938105805 & F & F & F\end{array}$ $\begin{array}{llllllllll}0.1111123692640206 & 0.5555522552209098 & 0.1750674087162309 & F & F & F\end{array}$ $\begin{array}{llllllll}0.4444440697289238 & 0.5555561254847916 & 0.1750719502190776 & F & F & F\end{array}$ $\begin{array}{llllllll}0.7777804782179913 & 0.5555742383122606 & 0.1750655488626904 & F & F & F\end{array}$ $\begin{array}{llllllll}0.1111199658994551 & 0.8888800192425492 & 0.1750494157144900 & F & F & F\end{array}$ $\begin{array}{llllllll}0.4444476042812084 & 0.8888872953412061 & 0.1750674087162309 & F & F & F\end{array}$ $\begin{array}{llllllll}0.7777970141657633 & 0.8888823414043898 & 0.1750651163386081 & F & F & F\end{array}$ 
$\begin{array}{lllllll}0.2242657109236099 & 0.1147251015225590 & 0.2662406435787559 & \mathrm{~T} & \mathrm{~T} & \mathrm{~T}\end{array}$ $\begin{array}{llllllll}0.5559152728827738 & 0.1114184951329684 & 0.2649560784399888 & \mathrm{~T} & \mathrm{~T} & \mathrm{~T}\end{array}$ $\begin{array}{llllllll}0.8893507309390879 & 0.1112338652890922 & 0.2649449497427928 & \mathrm{~T} & \mathrm{~T} & \mathrm{~T}\end{array}$ $\begin{array}{llllllll}0.2231657041214168 & 0.4426849531089395 & 0.2661203608252982 & \mathrm{~T} & \mathrm{~T} & \mathrm{~T}\end{array}$ $\begin{array}{lllllllll}0.5534008336331755 & 0.4427252045008263 & 0.2661080749670763 & \mathrm{~T} & \mathrm{~T} & \mathrm{~T}\end{array}$ $\begin{array}{llllllllll}0.8883266731557761 & 0.4426464547152027 & 0.2657269036039966 & \mathrm{~T} & \mathrm{~T} & \mathrm{~T}\end{array}$ $\begin{array}{llllllll}0.2226238104384674 & 0.7779303986206146 & 0.2650163407038440 & \mathrm{~T} & \mathrm{~T} & \mathrm{~T}\end{array}$ $\begin{array}{lllllll}0.5556129618196056 & 0.7774697063439479 & 0.2647568674476912 & \mathrm{~T} & \mathrm{~T} & \mathrm{~T}\end{array}$ $\begin{array}{llllllllllll}0.8889388590301562 & 0.7777916307773853 & 0.2650344723436049 & \text { T } & \text { T } & \text { T }\end{array}$ $\begin{array}{llllllllll}0.9972988004568093 & 0.9960997306624617 & 0.3489202525924624 & \mathrm{~T} & \mathrm{~T} & \mathrm{~T}\end{array}$ $\begin{array}{lllllllll}0.3326711423815232 & 0.9959869578779580 & 0.3489016987708813 & \mathrm{~T} & \mathrm{~T} & \mathrm{~T}\end{array}$ $\begin{array}{lllllllll}0.6664550229248549 & 0.9989413139613537 & 0.3485803938991679 & \mathrm{~T} & \mathrm{~T} & \mathrm{~T}\end{array}$ $\begin{array}{lllllllllll}0.0003128461354784 & 0.3321571013386502 & 0.3499255771399150 & \mathrm{~T} & \mathrm{~T} & \mathrm{~T}\end{array}$ $\begin{array}{llllllll}0.3320593871651816 & 0.3298816375976920 & 0.3569928226272741 & \mathrm{~T} & \mathrm{~T} & \mathrm{~T}\end{array}$ $\begin{array}{lllllllll}0.6658598791339840 & 0.3320507389521455 & 0.3499120630842615 & \mathrm{~T} & \mathrm{~T} & \mathrm{~T}\end{array}$ $\begin{array}{lllllllll}0.0000748973193884 & 0.6661571542581171 & 0.3493934096418259 & \mathrm{~T} & \mathrm{~T} & \mathrm{~T}\end{array}$ $\begin{array}{lllllllll}0.3343202682512865 & 0.6680272310599088 & 0.3489932784145139 & \mathrm{~T} & \mathrm{~T} & \mathrm{~T}\end{array}$ $\begin{array}{llllllll}0.6676973121139330 & 0.6680096907992878 & 0.3490000993260212 & \text { T } & \text { T } & \text { T }\end{array}$ $\begin{array}{llllllll}0.4284265155475125 & 0.3309302722960558 & 0.4414517587070718 & \mathrm{~T} & \mathrm{~T} & \mathrm{~T}\end{array}$ $\begin{array}{llllllll}0.2372048197215636 & 0.3306761892232316 & 0.4414734361550463 & \mathrm{~T} & \mathrm{~T} & \mathrm{~T}\end{array}$ $\begin{array}{lllllllll}0.2371699331481144 & 0.4724245277888496 & 0.4536418322165825 & \mathrm{~T} & \mathrm{~T} & \mathrm{~T}\end{array}$ $\begin{array}{llllllllll}0.0955770535965005 & 0.1886534700467737 & 0.4535871163198738 & \mathrm{~T} & \mathrm{~T} & \mathrm{~T}\end{array}$ $\begin{array}{llllllllll}0.5700033723657081 & 0.4728383250935478 & 0.4536239012162485 & \mathrm{~T} & \mathrm{~T} & \mathrm{~T}\end{array}$ $\begin{array}{llllllllll}0.4284399043032906 & 0.1891398594818928 & 0.4535802151577402 & \mathrm{~T} & \mathrm{~T} & \mathrm{~T}\end{array}$

22) $\mathrm{C}_{2} \mathrm{H}_{4}-\mathrm{PrO}_{2}-\mathrm{Co}(111)-3 \times 3$

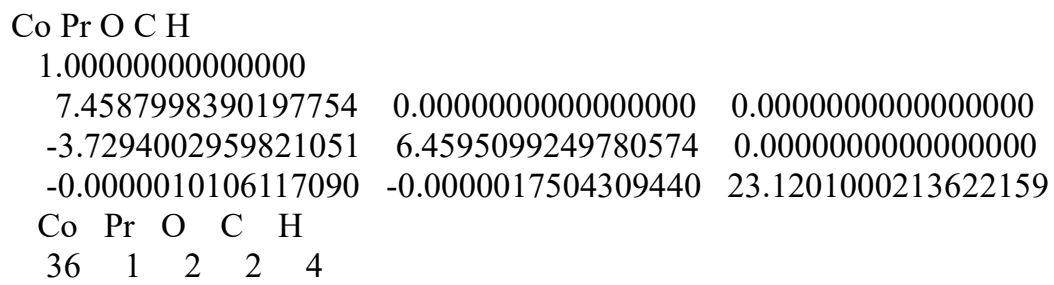

Selective dynamics

Direct

$\begin{array}{llllllll}0.0000353225881327 & 0.9999648942106703 & 0.0907110695049838 & F & F & F\end{array}$ $\begin{array}{llllllll}0.3333557427970177 & 0.0000001793936022 & 0.0907175141137841 & F & F & F\end{array}$ $\begin{array}{llllllll}0.6666917648931943 & 0.9999938437795350 & 0.0907267268766958 & F & F & F\end{array}$ $\begin{array}{llllllll}0.0000062578975388 & 0.33330843729885377 & 0.0907267268766958 & F & F & F\end{array}$ $\begin{array}{lllllllll}0.3333357007148621 & 0.3333426504206471 & 0.0907265971194704 & F & F & F\end{array}$ $\begin{array}{llllllll}0.6666945969032270 & 0.3333051862794676 & 0.0907313116319486 & F & F & F\end{array}$ $\begin{array}{llllllll}0.9999998791701969 & 0.6666444062796870 & 0.0907175141137841 & F & F & F\end{array}$ $\begin{array}{llllllll}0.3333385397113773 & 0.6666614354387903 & 0.0907302303217463 & F & F & F\end{array}$ $\begin{array}{lllllllll}0.6666573639132167 & 0.6666642220269168 & 0.0907265971194704 & F & F & F\end{array}$ $\begin{array}{llllllll}0.1111175321760314 & 0.2222028177229376 & 0.1750651163386081 & F & F & F\end{array}$ $\begin{array}{llllllll}0.4444257597051688 & 0.2222195372577005 & 0.1750655488626904 & \mathrm{~F} & \mathrm{~F} & \mathrm{~F}\end{array}$ $\begin{array}{llllllll}0.7777781372906531 & 0.2222218594174734 & 0.1750735938105805 & F & F & F\end{array}$ $\begin{array}{llllllll}0.1111123692640206 & 0.5555522552209098 & 0.1750674087162309 & F & F & F\end{array}$ $\begin{array}{llllllll}0.4444440697289238 & 0.5555561254847916 & 0.1750719502190776 & F & F & F\end{array}$ $\begin{array}{lllllll}0.7777804782179913 & 0.5555742383122606 & 0.1750655488626904 & F & F & F\end{array}$ $\begin{array}{llllllll}0.1111199658994551 & 0.8888800192425492 & 0.1750494157144900 & F & F & F\end{array}$ $\begin{array}{llllllll}0.4444476042812084 & 0.8888872953412061 & 0.1750674087162309 & F & F & F\end{array}$ $\begin{array}{llllllll}0.7777970141657633 & 0.8888823414043898 & 0.1750651163386081 & F & F & F\end{array}$ 
$\begin{array}{lllllll}0.2209980464308831 & 0.1101994349018439 & 0.2643889192036905 & \mathrm{~T} & \mathrm{~T} & \mathrm{~T}\end{array}$ $\begin{array}{lllllllll}0.5571728998362104 & 0.1160001257901519 & 0.2663384997830477 & \mathrm{~T} & \mathrm{~T} & \mathrm{~T}\end{array}$ $\begin{array}{llllllll}0.8892692583318079 & 0.1126869649624241 & 0.2647303396849227 & \mathrm{~T} & \mathrm{~T} & \mathrm{~T}\end{array}$ $\begin{array}{lllllll}0.2174945364062416 & 0.4471494546774587 & 0.2630309179290135 & \mathrm{~T} & \mathrm{~T} & \mathrm{~T}\end{array}$ $\begin{array}{lllllllll}0.5603716516117682 & 0.4425085633511537 & 0.2646716898292288 & \mathrm{~T} & \mathrm{~T} & \mathrm{~T}\end{array}$ $\begin{array}{lllllll}0.8847515255389152 & 0.4432257747752574 & 0.2685312444473849 & \mathrm{~T} & \mathrm{~T} & \mathrm{~T}\end{array}$ $\begin{array}{lllllll}0.2196055993189893 & 0.7760230650943726 & 0.2658843629749298 & \mathrm{~T} & \mathrm{~T} & \mathrm{~T}\end{array}$ $\begin{array}{llllllll}0.5552148444801831 & 0.7788503566162986 & 0.2653015789961129 & \mathrm{~T} & \mathrm{~T} & \mathrm{~T}\end{array}$ $\begin{array}{llllllll}0.8893161660892615 & 0.7776163425244919 & 0.2651935432038988 & \mathrm{~T} & \mathrm{~T} & \mathrm{~T}\end{array}$ $\begin{array}{llllllll}0.9964786908201744 & 0.9965446779370216 & 0.3505508526578509 & \mathrm{~T} & \mathrm{~T} & \mathrm{~T}\end{array}$ $\begin{array}{lllllll}0.3355403809997986 & -0.0015604060269711 & 0.3491398114134163 & \mathrm{~T} & \mathrm{~T} & \mathrm{~T}\end{array}$ $\begin{array}{lllllllll}0.6693899748280869 & 0.0023834430708399 & 0.3488177242657231 & \mathrm{~T} & \mathrm{~T} & \mathrm{~T}\end{array}$ $\begin{array}{lllllll}0.0041395945005226 & 0.3335035398073463 & 0.3510107938094524 & \mathrm{~T} & \mathrm{~T} & \mathrm{~T}\end{array}$ $\begin{array}{lllllll}0.3386463232875401 & 0.3427986816300322 & 0.3440863593790486 & \mathrm{~T} & \mathrm{~T} & \mathrm{~T}\end{array}$ $\begin{array}{llllllll}0.6681912492762042 & 0.3305418403298164 & 0.3676496887915006 & \mathrm{~T} & \mathrm{~T} & \mathrm{~T}\end{array}$ $\begin{array}{llllllll}0.0000151513921072 & 0.6729164347542672 & 0.3528951763938990 & \mathrm{~T} & \mathrm{~T} & \mathrm{~T}\end{array}$ $\begin{array}{lllllllll}0.3408136279672705 & 0.6730025336831724 & 0.3522819889174637 & \mathrm{~T} & \mathrm{~T} & \mathrm{~T}\end{array}$ $\begin{array}{lllllllll}0.6679794863839531 & 0.6662159739785040 & 0.3478501928347509 & \mathrm{~T} & \mathrm{~T} & \mathrm{~T}\end{array}$ $\begin{array}{llllllll}0.3058716848368546 & 0.2222135229001010 & 0.4506349538699512 & \mathrm{~T} & \mathrm{~T} & \mathrm{~T}\end{array}$ $\begin{array}{llllllll}0.2257725315690227 & 0.4499629951014685 & 0.4099864774721197 & \mathrm{~T} & \mathrm{~T} & \mathrm{~T}\end{array}$ $\begin{array}{llllllll}0.1179135352789193 & 0.9059269360625436 & 0.4128224797893326 & \mathrm{~T} & \mathrm{~T} & \mathrm{~T}\end{array}$ $\begin{array}{llllllll}0.7615154663577275 & 0.4542228494073270 & 0.4477574044383761 & \mathrm{~T} & \mathrm{~T} & \mathrm{~T}\end{array}$ $\begin{array}{llllllll}0.6995290885957068 & 0.2372066396317101 & 0.4481518879008186 & \mathrm{~T} & \mathrm{~T} & \mathrm{~T}\end{array}$ $\begin{array}{llllllll}0.6712986255416902 & 0.5147344354222005 & 0.4701991526925626 & \mathrm{~T} & \mathrm{~T} & \mathrm{~T}\end{array}$ $\begin{array}{llllllll}0.9259530317460827 & 0.5676923488800137 & 0.4531614619070097 & \mathrm{~T} & \mathrm{~T} & \mathrm{~T}\end{array}$ $\begin{array}{lllllll}0.5648201084523778 & 0.1191885474610434 & 0.4729009363651036 & \mathrm{~T} & \mathrm{~T} & \mathrm{~T}\end{array}$ $\begin{array}{lllllll}0.8220946084255338 & 0.1977725575493214 & 0.4518552046343371 & \mathrm{~T} & \mathrm{~T} & \mathrm{~T}\end{array}$

23) $\mathrm{C}_{2} \mathrm{H}_{4}-\mathrm{Co}(211)-1 \times 3$

$\mathrm{Co} \mathrm{C} \mathrm{H}$

1.00000000000000

$\begin{array}{llll}6.0968000000000000 & 0.0000000000000000 & 0.0000000000000000\end{array}$

$\begin{array}{llll}0.0000000000000000 & 7.4669999999999996 & 0.0000000000000000\end{array}$

$\begin{array}{llll}0.0000000000000000 & 0.0000000000000000 & 23.6221999999999994\end{array}$

$\mathrm{Co} \mathrm{C} \quad \mathrm{H}$

$\begin{array}{lll}48 & 2 & 4\end{array}$

Selective dynamics

Direct

$\begin{array}{llllllll}0.0039768403096687 & 0.1666663988214836 & 0.9745822997011260 & F & F & F\end{array}$ $\begin{array}{lllllllll}0.0039765122687285 & 0.5000000000000000 & 0.9745822997011260 & F & F & F\end{array}$ $\begin{array}{lllllllll}0.0039768403096687 & 0.8333336011785164 & 0.9745822997011260 & F & F & F\end{array}$ $\begin{array}{lllllllll}0.3315888662905095 & 0.0000000000000000 & 0.0034278771663949 & F & F & F\end{array}$ $\begin{array}{lllllllll}0.3315855858811148 & 0.3333350743270387 & 0.0034291471581795 & F & F & F\end{array}$ $\begin{array}{llllllll}0.3315855858811148 & 0.6666647917503710 & 0.0034291471581795 & F & F & F\end{array}$ $\begin{array}{llllllll}0.6664853693741009 & 0.1666655952859273 & 0.0285661792720404 & F & F & F\end{array}$ $\begin{array}{llllllll}0.6664884857630256 & 0.5000000000000000 & 0.0285654596100287 & F & F & F\end{array}$ $\begin{array}{lllllllll}0.6664853693741009 & 0.8333344047140727 & 0.0285661792720404 & F & F & F\end{array}$ $\begin{array}{lllllllll}0.9983825941477491 & 0.0000000000000000 & 0.0609119811025209 & F & F & F\end{array}$ $\begin{array}{llllllll}0.9983791497178842 & 0.3333345386366702 & 0.0609079171288016 & F & F & F\end{array}$ $\begin{array}{llllllll}0.9983791497178842 & 0.6666654613633298 & 0.0609079171288016 & F & F & F\end{array}$ $\begin{array}{llllllll}0.3314264860254568 & 0.1666650595955517 & 0.0918800111759310 & F & F & F\end{array}$ $\begin{array}{lllllllll}0.3314281262301506 & 0.5000000000000000 & 0.0918805615057039 & F & F & F\end{array}$ $\begin{array}{llllllll}0.3314264860254568 & 0.8333349404044483 & 0.0918800111759310 & F & F & F\end{array}$ 
$\begin{array}{llllllll}0.6654751673008761 & 0.0000000000000000 & 0.1205095207050988 & F & F & F\end{array}$ $\begin{array}{llllllll}0.6654631938065876 & 0.3333330654881479 & 0.1205040597404121 & F & F & F\end{array}$ $\begin{array}{llllllll}0.6654631938065876 & 0.6666669345118521 & 0.1205040597404121 & F & F & F\end{array}$ $\begin{array}{llllllll}0.0009121178323070 & 0.1666669345118521 & 0.1526111031148645 & F & F & F\end{array}$ $\begin{array}{llllllll}0.0009114617504267 & 0.5000000000000000 & 0.1526112301140472 & F & F & F\end{array}$ $\begin{array}{lllllll}0.0009121178323070 & 0.8333330654881479 & 0.1526111031148645 & F & F & F\end{array}$ $\begin{array}{llllllll}0.3340214538774404 & 0.0000000000000000 & 0.1833969740329024 & F & F & F\end{array}$ $\begin{array}{llllllll}0.3340229300616713 & 0.3333327976429601 & 0.1833968470337197 & F & F & F\end{array}$ $\begin{array}{llllllll}0.3340229300616713 & 0.6666672023570399 & 0.1833968470337197 & F & F & F\end{array}$ $\begin{array}{lllllllll}0.6659255956148380 & 0.1668387306927896 & 0.2118315399214045 & \mathrm{~T} & \mathrm{~T} & \mathrm{~T}\end{array}$ $\begin{array}{llllllll}0.6653797029869145 & 0.5001838278721612 & 0.2118774771242978 & \mathrm{~T} & \mathrm{~T} & \mathrm{~T}\end{array}$ $\begin{array}{lllllllll}0.6658769680766116 & 0.8336309042963843 & 0.2118161480787779 & \mathrm{~T} & \mathrm{~T} & \mathrm{~T}\end{array}$ $\begin{array}{lllllll}0.9984971450770040 & 0.0000574930443549 & 0.2427842079845912 & \mathrm{~T} & \mathrm{~T} & \mathrm{~T}\end{array}$ $\begin{array}{lllllll}0.9986445787685340 & 0.3335318149466585 & 0.2426302729555652 & \mathrm{~T} & \mathrm{~T} & \mathrm{~T}\end{array}$ $\begin{array}{llllllll}0.9984413789950910 & 0.6667999211793522 & 0.2426858600025979 & \mathrm{~T} & \mathrm{~T} & \mathrm{~T}\end{array}$ $\begin{array}{lllllllll}0.3333571390927925 & 0.1668517437295304 & 0.2746159989675913 & \mathrm{~T} & \mathrm{~T} & \mathrm{~T}\end{array}$ $\begin{array}{llllllllll}0.3331810360535596 & 0.5000418677656948 & 0.2748497977692068 & \mathrm{~T} & \mathrm{~T} & \mathrm{~T}\end{array}$ $\begin{array}{llllllll}0.3329914746820734 & 0.8335033693635592 & 0.2745832478074934 & \mathrm{~T} & \mathrm{~T} & \mathrm{~T}\end{array}$ $\begin{array}{llllllllll}0.6678810436205741 & 0.0005282621480566 & 0.3033322433560609 & \text { T } & \text { T } & \text { T }\end{array}$ $\begin{array}{lllllllll}0.6671233901051468 & 0.3338943697410655 & 0.3031890412687036 & \mathrm{~T} & \mathrm{~T} & \mathrm{~T}\end{array}$ $\begin{array}{llllllllll}0.6671154769249358 & 0.6676688706018699 & 0.3031573217065783 & \mathrm{~T} & \mathrm{~T} & \mathrm{~T}\end{array}$ $\begin{array}{llllllll}-0.0006674880643545 & 0.1672270440010111 & 0.3342881213233195 & \text { T } & \text { T } & \text { T }\end{array}$ $\begin{array}{llllllllll}-0.0016085515181861 & 0.5012763576783755 & 0.3343507484806075 & \mathrm{~T} & \mathrm{~T} & \mathrm{~T}\end{array}$ $\begin{array}{llllllllll}-0.0000178191281885 & 0.8334978938004244 & 0.3347584239254892 & \text { T } & \text { T } & \text { T }\end{array}$ $\begin{array}{lllllll}0.3323622929447293 & 0.0002567776546732 & 0.3657842483976530 & \mathrm{~T} & \mathrm{~T} & \mathrm{~T}\end{array}$ $\begin{array}{llllllll}0.3305193357987513 & 0.3327872460604484 & 0.3655628744050484 & \mathrm{~T} & \mathrm{~T} & \mathrm{~T}\end{array}$ $\begin{array}{llllllll}0.3300943191476738 & 0.6686190141794206 & 0.3659173082467139 & \mathrm{~T} & \mathrm{~T} & \mathrm{~T}\end{array}$ $\begin{array}{llllllll}0.6684556918528178 & 0.1674027253918159 & 0.3913955608848111 & \mathrm{~T} & \mathrm{~T} & \mathrm{~T}\end{array}$ $\begin{array}{llllllllll}0.6625703267113854 & 0.5011223272635696 & 0.3903764311001696 & \mathrm{~T} & \mathrm{~T} & \mathrm{~T}\end{array}$ $\begin{array}{lllllllllll}0.6693267388840644 & 0.8341963672512018 & 0.3917399749298996 & \mathrm{~T} & \mathrm{~T} & \mathrm{~T}\end{array}$ $\begin{array}{llllllllll}-0.0008281108981059 & 0.0016259692177587 & 0.4205329358204484 & \mathrm{~T} & \mathrm{~T} & \mathrm{~T}\end{array}$ $\begin{array}{llllllll}0.9875622956403196 & 0.3334631624437212 & 0.4235863539587836 & \mathrm{~T} & \mathrm{~T} & \mathrm{~T}\end{array}$ $\begin{array}{llllllllll}0.9898947609429594 & 0.6628118333604518 & 0.4224832592435809 & \text { T } & \text { T } & \text { T }\end{array}$ $\begin{array}{llllllll}0.0197522740231572 & 0.4870416862683511 & 0.4968718156490633 & \mathrm{~T} & \mathrm{~T} & \mathrm{~T}\end{array}$ $\begin{array}{lllllll}0.0999041887092184 & 0.3063038385448135 & 0.5024307191912123 & \mathrm{~T} & \mathrm{~T} & \mathrm{~T}\end{array}$ $\begin{array}{lllllllll}0.8665537210404497 & 0.5207644437269070 & 0.5184790034283027 & \mathrm{~T} & \mathrm{~T} & \mathrm{~T}\end{array}$ $\begin{array}{llllllll}0.1451789543060471 & 0.5952989928720477 & 0.4989389199443363 & \mathrm{~T} & \mathrm{~T} & \mathrm{~T}\end{array}$ $\begin{array}{lllllll}0.0067371951531023 & 0.2121398240844097 & 0.5284802670913448 & \mathrm{~T} & \mathrm{~T} & \mathrm{~T}\end{array}$ $\begin{array}{llllllllll}0.2765961606817176 & 0.2823001321281038 & 0.5014026261389113 & \mathrm{~T} & \mathrm{~T} & \mathrm{~T}\end{array}$

24) $\mathrm{C}_{2} \mathrm{H}_{4}-\mathrm{PrO}_{2}-\mathrm{Co}(211)-1 \times 3$

Co Pr O C H

1.00000000000000

$\begin{array}{llll}6.0968000000000000 & 0.0000000000000000 & 0.0000000000000000\end{array}$

$\begin{array}{llll}0.0000000000000000 & 7.46699999999999996 & 0.0000000000000000\end{array}$

$\begin{array}{llll}0.0000000000000000 & 0.0000000000000000 & 23.62219999999999994\end{array}$

Co $\begin{array}{lllll} & \mathrm{O} & \mathrm{C} & \mathrm{H}\end{array}$

$\begin{array}{lllll}48 & 1 & 2 & 2 & 4\end{array}$

Selective dynamics

Direct

$\begin{array}{llllllll}0.0039768400000000 & 0.1666663990000004 & 0.9745823000000016 & F & F & F\end{array}$ $\begin{array}{llllllll}0.0039765120000013 & 0.5000000000000000 & 0.9745823000000016 & F & F & F\end{array}$ $\begin{array}{llllllll}0.0039768400000000 & 0.83333336009999996 & 0.9745823000000016 & F & F & F\end{array}$ 
$\begin{array}{llllllll}0.3315888659999970 & 0.0000000000000000 & 0.0034278770000000 & F & F & F\end{array}$ $\begin{array}{llllllll}0.3315855860000028 & 0.33333507399999972 & 0.0034291469999985 & \mathrm{~F} & \mathrm{~F} & \mathrm{~F}\end{array}$ $\begin{array}{llllllllll}0.3315855860000028 & 0.6666647919999988 & 0.0034291469999985 & \mathrm{~F} & \mathrm{~F} & \mathrm{~F}\end{array}$ $\begin{array}{lllllllll}0.6664853690000001 & 0.1666655949999978 & 0.0285661790000020 & F & F & F\end{array}$ $\begin{array}{llllllllll}0.6664884859999987 & 0.5000000000000000 & 0.0285654600000029 & F & F & F\end{array}$ $\begin{array}{llllllll}0.6664853690000001 & 0.8333344050000022 & 0.0285661790000020 & F & F & F\end{array}$ $\begin{array}{lllllllll}0.9983825939999988 & 0.0000000000000000 & 0.0609119809999967 & F & F & F\end{array}$ $\begin{array}{llllllll}0.9983791499999981 & 0.3333345389999991 & 0.0609079170000015 & F & F & F\end{array}$ $\begin{array}{llllllll}0.9983791499999981 & 0.6666654610000009 & 0.0609079170000015 & F & F & F\end{array}$ $\begin{array}{llllllll}0.3314264859999980 & 0.1666650599999997 & 0.0918800110000006 & F & F & F\end{array}$ $\begin{array}{lllllllll}0.3314281259999987 & 0.5000000000000000 & 0.0918805620000001 & F & F & F\end{array}$ $\begin{array}{llllllll}0.3314264859999980 & 0.83333449400000003 & 0.0918800110000006 & F & F & F\end{array}$ $\begin{array}{llllllll}0.6654751669999968 & 0.0000000000000000 & 0.1205095210000025 & F & F & F\end{array}$ $\begin{array}{llllllll}0.6654631939999973 & 0.3333330649999979 & 0.1205040600000018 & F & F & F\end{array}$ $\begin{array}{llllllll}0.6654631939999973 & 0.6666669350000021 & 0.1205040600000018 & F & F & F\end{array}$ $\begin{array}{llllllllll}0.0009121180000022 & 0.1666669350000021 & 0.1526111029999981 & F & F & F\end{array}$ $\begin{array}{lllllllll}0.0009114619999977 & 0.5000000000000000 & 0.1526112299999980 & F & F & F\end{array}$ $\begin{array}{llllllll}0.0009121180000022 & 0.8333330649999979 & 0.1526111029999981 & F & F & F\end{array}$ $\begin{array}{lllllll}0.3340214540000019 & 0.0000000000000000 & 0.18339697399999972 & F & F & F\end{array}$ $\begin{array}{lllllll}0.3340229300000033 & 0.3333327980000007 & 0.18339684699999974 & F & F & F\end{array}$ $\begin{array}{llllllll}0.3340229300000033 & 0.6666672019999993 & 0.18339684699999974 & F & F & F\end{array}$ $\begin{array}{llllllll}0.6645038679418933 & 0.1666940828473601 & 0.2117536149345588 & \mathrm{~T} & \mathrm{~T} & \mathrm{~T}\end{array}$ $\begin{array}{lllllllll}0.6668350980161388 & 0.4999274581149993 & 0.2118850651104055 & \mathrm{~T} & \mathrm{~T} & \mathrm{~T}\end{array}$ $\begin{array}{llllllll}0.6666925967495559 & 0.8345085471319806 & 0.2116843195144681 & \mathrm{~T} & \mathrm{~T} & \mathrm{~T}\end{array}$ $\begin{array}{llllllll}0.9971402464938849 & 0.0018831507653378 & 0.2433410364505671 & \mathrm{~T} & \mathrm{~T} & \mathrm{~T}\end{array}$ $\begin{array}{lllllll}0.9991928755267638 & 0.3331416442830909 & 0.2431702507789253 & \mathrm{~T} & \mathrm{~T} & \mathrm{~T}\end{array}$ $\begin{array}{lllllll}0.0008537951422136 & 0.6668538866507475 & 0.2429867945952075 & \mathrm{~T} & \mathrm{~T} & \mathrm{~T}\end{array}$ $\begin{array}{llllllll}0.3309792965735021 & 0.1668836570767019 & 0.2762180080254098 & \mathrm{~T} & \mathrm{~T} & \mathrm{~T}\end{array}$ $\begin{array}{lllllllll}0.3354938905205690 & 0.4977321036000936 & 0.2750810653308575 & \mathrm{~T} & \mathrm{~T} & \mathrm{~T}\end{array}$ $\begin{array}{lllllllll}0.3328659922245464 & 0.8353508127889034 & 0.2731939475909700 & \mathrm{~T} & \mathrm{~T} & \mathrm{~T}\end{array}$ $\begin{array}{llllllllll}0.6652536569758811 & 0.0021836096516317 & 0.3033413720717101 & \mathrm{~T} & \mathrm{~T} & \mathrm{~T}\end{array}$ $\begin{array}{lllllll}0.6682678429565183 & 0.3294490379773405 & 0.3024289597817802 & \mathrm{~T} & \mathrm{~T} & \mathrm{~T}\end{array}$ $\begin{array}{llllllll}0.6679892472703765 & 0.6679349043341912 & 0.3021192465686219 & \mathrm{~T} & \mathrm{~T} & \mathrm{~T}\end{array}$ $\begin{array}{llllllll}0.9965622463935024 & 0.1649853497978349 & 0.3367881522626946 & \mathrm{~T} & \mathrm{~T} & \mathrm{~T}\end{array}$ $\begin{array}{lllllllll}0.0046695673829341 & 0.4967309628013341 & 0.3347662478787963 & \mathrm{~T} & \mathrm{~T} & \mathrm{~T}\end{array}$ $\begin{array}{llllllll}0.9990759922421457 & 0.8341797943545636 & 0.3341583858793399 & \mathrm{~T} & \mathrm{~T} & \mathrm{~T}\end{array}$ $\begin{array}{lllllll}0.3290719607093087 & 0.9949971320067698 & 0.3656680967764716 & \mathrm{~T} & \mathrm{~T} & \mathrm{~T}\end{array}$ $\begin{array}{llllllll}0.3237480608560939 & 0.3314327535249652 & 0.3698997535807703 & \mathrm{~T} & \mathrm{~T} & \mathrm{~T}\end{array}$ $\begin{array}{llllllllll}0.3292654083547463 & 0.6733201621147146 & 0.3621776736307801 & \mathrm{~T} & \mathrm{~T} & \mathrm{~T}\end{array}$ $\begin{array}{llllllllll}0.6665080733917150 & 0.1624685163243748 & 0.3960146142925514 & \mathrm{~T} & \mathrm{~T} & \mathrm{~T}\end{array}$ $\begin{array}{llllllllll}0.6701042555922035 & 0.5001995875147793 & 0.3874395505083134 & \mathrm{~T} & \mathrm{~T} & \mathrm{~T}\end{array}$ $\begin{array}{llllllll}0.6692529831814400 & 0.8377081214686597 & 0.3907932855460702 & \mathrm{~T} & \mathrm{~T} & \mathrm{~T}\end{array}$ $\begin{array}{llllllll}0.0025664233099282 & 0.9943275746622604 & 0.4272845971575843 & \mathrm{~T} & \mathrm{~T} & \mathrm{~T}\end{array}$ $\begin{array}{llllllll}0.9922653008024535 & 0.3348866262701052 & 0.4282177828007835 & \mathrm{~T} & \mathrm{~T} & \mathrm{~T}\end{array}$ $\begin{array}{llllllll}0.9751474400853127 & 0.6664751578039481 & 0.4210454795279717 & \mathrm{~T} & \mathrm{~T} & \mathrm{~T}\end{array}$ $\begin{array}{llllllll}0.3991738023863956 & 0.6092152523544858 & 0.4802479845734832 & \mathrm{~T} & \mathrm{~T} & \mathrm{~T}\end{array}$ $\begin{array}{llllllllll}0.5112790969425469 & 0.3452239070094069 & 0.4413606668151314 & \mathrm{~T} & \mathrm{~T} & \mathrm{~T}\end{array}$ $\begin{array}{llllllll}0.0507852023145833 & 0.5234423790341352 & 0.4843074240083908 & \mathrm{~T} & \mathrm{~T} & \mathrm{~T}\end{array}$ $\begin{array}{lllllllll}0.0113607856367539 & 0.1124415743390720 & 0.5055094849266789 & \mathrm{~T} & \mathrm{~T} & \mathrm{~T}\end{array}$ $\begin{array}{llllllll}0.1364485176296854 & 0.9503261775230339 & 0.5047320090506463 & \mathrm{~T} & \mathrm{~T} & \mathrm{~T}\end{array}$ $\begin{array}{llllllll}0.8478033399128052 & 0.1115665824968311 & 0.5243567157988805 & \text { T } & \text { T } & \text { T }\end{array}$ $\begin{array}{llllllll}0.0989325875189479 & 0.2386892141042800 & 0.5124338374812948 & \mathrm{~T} & \mathrm{~T} & \mathrm{~T}\end{array}$ $\begin{array}{llllllll}0.0719028159203490 & 0.8320885251454846 & 0.5264880555014494 & \mathrm{~T} & \mathrm{~T} & \mathrm{~T}\end{array}$ $\begin{array}{lllllllll}0.3159513489639311 & 0.9670478813435246 & 0.5044756896820537 & \mathrm{~T} & \mathrm{~T} & \mathrm{~T}\end{array}$ 
25) $\mathrm{C}-\mathrm{Co}(111)-3 \times 3$

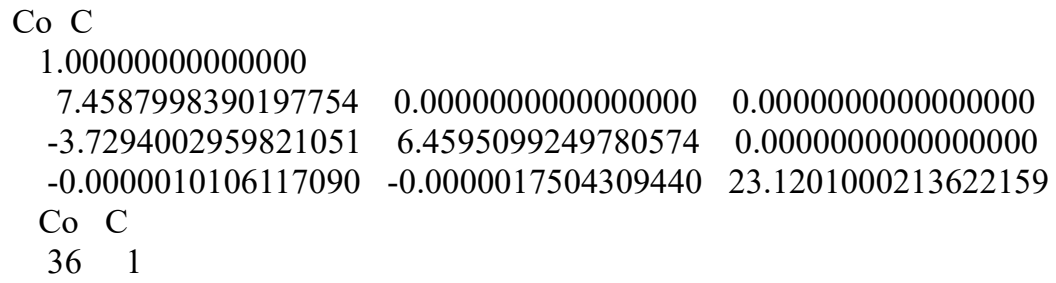

Co $\operatorname{Pr} \mathrm{C}$

1.00000000000000 


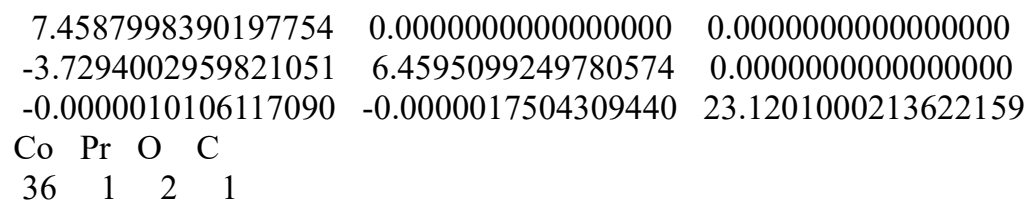

Selective dynamics

Direct

$\begin{array}{llllllll}0.0000353225881327 & 0.9999648942106703 & 0.0907110695049766 & F & F & F\end{array}$ $\begin{array}{lllllllll}0.3333557427970177 & 0.0000001793936022 & 0.0907175141137770 & F & F & F\end{array}$ $\begin{array}{lllllllll}0.6666917648932014 & 0.9999938437795421 & 0.0907267268767029 & F & F & F\end{array}$ $\begin{array}{llllllllll}0.0000062578975388 & 0.3333084372988466 & 0.0907267268767029 & \mathrm{~F} & \mathrm{~F} & \mathrm{~F}\end{array}$ $\begin{array}{lllllllll}0.3333357007148621 & 0.3333426504206471 & 0.0907265971194704 & F & F & F\end{array}$ $\begin{array}{llllllll}0.6666945969032270 & 0.3333051862794676 & 0.0907313116319486 & F & F & F\end{array}$ $\begin{array}{llllllll}0.9999998791701969 & 0.6666444062796870 & 0.0907175141137770 & F & F & F\end{array}$ $\begin{array}{lllllllll}0.3333385397113773 & 0.6666614354387903 & 0.0907302303217534 & F & F & F\end{array}$ $\begin{array}{lllllllll}0.6666573639132167 & 0.6666642220269168 & 0.0907265971194704 & F & F & F\end{array}$ $\begin{array}{llllllll}0.1111175321760314 & 0.2222028177229376 & 0.1750651163386081 & F & F & F\end{array}$ $\begin{array}{lllllllll}0.4444257597051688 & 0.2222195372577005 & 0.1750655488626904 & F & F & F\end{array}$ $\begin{array}{llllllll}0.7777781372906531 & 0.2222218594174734 & 0.1750735938105805 & F & F & F\end{array}$ $\begin{array}{lllllll}0.1111123692640206 & 0.5555522552209098 & 0.1750674087162309 & F & F & F\end{array}$ $\begin{array}{llllllll}0.4444440697289167 & 0.5555561254847916 & 0.1750719502190776 & F & F & F\end{array}$ $\begin{array}{lllllllll}0.7777804782179913 & 0.5555742383122606 & 0.1750655488626904 & F & F & F\end{array}$ $\begin{array}{llllllll}0.1111199658994622 & 0.8888800192425492 & 0.1750494157144900 & F & F & F\end{array}$ $\begin{array}{lllllll}0.4444476042812084 & 0.8888872953412132 & 0.1750674087162309 & F & F & F\end{array}$ $\begin{array}{lllllll}0.7777970141657633 & 0.8888823414043898 & 0.1750651163386081 & F & F & F\end{array}$ $\begin{array}{lllllllll}0.2218311591948475 & 0.1117782956357841 & 0.2658927108738715 & \mathrm{~T} & \mathrm{~T} & \mathrm{~T}\end{array}$ $\begin{array}{llllllll}0.5520512691347320 & 0.1087812062587375 & 0.2640215454555646 & \mathrm{~T} & \mathrm{~T} & \mathrm{~T}\end{array}$ $\begin{array}{lllllllll}0.8880998421085202 & 0.1115113265673815 & 0.2648052409722573 & \mathrm{~T} & \mathrm{~T} & \mathrm{~T}\end{array}$ $\begin{array}{lllllllll}0.2181074737776463 & 0.4452995232789953 & 0.2641144692151559 & \mathrm{~T} & \mathrm{~T} & \mathrm{~T}\end{array}$ $\begin{array}{llllllll}0.5567065898040164 & 0.4446309801731888 & 0.2634300597465954 & \mathrm{~T} & \mathrm{~T} & \mathrm{~T}\end{array}$ $\begin{array}{llllllll}0.8880566485227415 & 0.4446277832635925 & 0.2659673855499142 & \mathrm{~T} & \mathrm{~T} & \mathrm{~T}\end{array}$ $\begin{array}{lllllll}0.2184466051679525 & 0.7754253325626249 & 0.2668619563035719 & \mathrm{~T} & \mathrm{~T} & \mathrm{~T}\end{array}$ $\begin{array}{llllllll}0.5511384030787515 & 0.7751132443535852 & 0.2651419494566352 & \mathrm{~T} & \mathrm{~T} & \mathrm{~T}\end{array}$ $\begin{array}{llllllllll}0.8884637831048160 & 0.7760784028668397 & 0.2656912620923639 & \mathrm{~T} & \mathrm{~T} & \mathrm{~T}\end{array}$ $\begin{array}{lllllllll}0.9951435056618591 & 0.9965923396727504 & 0.3509055192278500 & \mathrm{~T} & \mathrm{~T} & \mathrm{~T}\end{array}$ $\begin{array}{lllllllll}0.3341710525199230 & 0.9954714925993028 & 0.3513399207434024 & \mathrm{~T} & \mathrm{~T} & \mathrm{~T}\end{array}$ $\begin{array}{llllllll}0.6643910092134667 & 0.9974657412287999 & 0.3482107449411130 & \text { T } & \text { T } & \text { T }\end{array}$ $\begin{array}{llllllll}0.9905080561891485 & 0.3270842645988612 & 0.3521765925436749 & \text { T } & \text { T } & \text { T }\end{array}$ $\begin{array}{llllllllll}0.3396942319454552 & 0.3434452599255863 & 0.3486861777846760 & \mathrm{~T} & \mathrm{~T} & \mathrm{~T}\end{array}$ $\begin{array}{llllllllll}0.6684940519241113 & 0.3238963528559608 & 0.3505221185128853 & \mathrm{~T} & \mathrm{~T} & \mathrm{~T}\end{array}$ $\begin{array}{llllllllll}0.9977726539832280 & 0.6704269371463879 & 0.3541421147737177 & \mathrm{~T} & \mathrm{~T} & \mathrm{~T}\end{array}$ $\begin{array}{llllllll}0.3338816009647281 & 0.6707543357552881 & 0.3524058768567013 & \mathrm{~T} & \mathrm{~T} & \mathrm{~T}\end{array}$ $\begin{array}{llllllllll}0.6698492751024521 & 0.6749375720923321 & 0.3508546773564742 & \mathrm{~T} & \mathrm{~T} & \mathrm{~T}\end{array}$ $\begin{array}{llllllll}0.2649230726534089 & 0.2120761078475647 & 0.4554303012850158 & \mathrm{~T} & \mathrm{~T} & \mathrm{~T}\end{array}$ $\begin{array}{lllllllll}0.2018562951935000 & 0.4433604389839184 & 0.4096996066621731 & \mathrm{~T} & \mathrm{~T} & \mathrm{~T}\end{array}$ $\begin{array}{lllllllll}0.1178049541586798 & 0.9004060786490327 & 0.4125482736580846 & \mathrm{~T} & \mathrm{~T} & \mathrm{~T}\end{array}$ $\begin{array}{lllllllllll}0.5817109227011624 & 0.4528338648051564 & 0.3978203662973674 & \mathrm{~T} & \mathrm{~T} & \mathrm{~T}\end{array}$

27) $\mathrm{C}-\mathrm{Na}_{2} \mathrm{O}-\mathrm{Co}(111)-3 \times 3$

$\mathrm{Co} \mathrm{O} \mathrm{NaC}$ 1.00000000000000 7.4587998390197754 $-3.7294002959821051$

$0.0000000000000000 \quad 0.0000000000000000$ $\begin{array}{lll}6.4595099249780574 & 0.0000000000000000\end{array}$ 


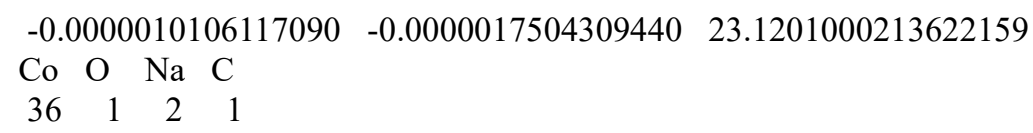

Selective dynamics

Direct

$\begin{array}{lllllll}0.0000353225881327 & 0.9999648942106703 & 0.0907110695049766 & \mathrm{~F} & \mathrm{~F} & \mathrm{~F}\end{array}$ $\begin{array}{llllllllll}0.3333557427970177 & 0.0000001793936022 & 0.0907175141137770 & \mathrm{~F} & \mathrm{~F} & \mathrm{~F}\end{array}$ $\begin{array}{llllllll}0.6666917648932014 & 0.9999938437795421 & 0.0907267268767029 & F & F & F\end{array}$ $\begin{array}{llllllll}0.0000062578975388 & 0.3333084372988466 & 0.0907267268767029 & F & F & F\end{array}$ $\begin{array}{lllllllll}0.3333357007148621 & 0.3333426504206471 & 0.0907265971194704 & F & F & F\end{array}$ $\begin{array}{llllllllll}0.6666945969032270 & 0.3333051862794676 & 0.0907313116319486 & F & F & F\end{array}$ $\begin{array}{llllllllll}0.9999998791701969 & 0.6666444062796870 & 0.0907175141137770 & F & F & F\end{array}$ $\begin{array}{lllllllll}0.3333385397113773 & 0.6666614354387903 & 0.0907302303217534 & F & F & F\end{array}$ $\begin{array}{lllllllll}0.6666573639132167 & 0.6666642220269168 & 0.0907265971194704 & F & F & F\end{array}$ $\begin{array}{llllllllll}0.1111175321760314 & 0.2222028177229376 & 0.1750651163386081 & F & F & F\end{array}$ $\begin{array}{llllllll}0.4444257597051688 & 0.2222195372577005 & 0.1750655488626904 & F & F & F\end{array}$ $\begin{array}{llllllll}0.7777781372906531 & 0.2222218594174734 & 0.1750735938105805 & F & F & F\end{array}$ $\begin{array}{llllllllll}0.1111123692640206 & 0.5555522552209098 & 0.1750674087162309 & F & F & F\end{array}$ $\begin{array}{lllllll}0.4444440697289167 & 0.5555561254847916 & 0.1750719502190776 & F & F & F\end{array}$ $\begin{array}{llllllll}0.7777804782179913 & 0.5555742383122606 & 0.1750655488626904 & F & F & F\end{array}$ $\begin{array}{lllllll}0.1111199658994622 & 0.8888800192425492 & 0.1750494157144900 & F & F & F\end{array}$ $\begin{array}{lllllll}0.4444476042812084 & 0.8888872953412132 & 0.1750674087162309 & F & F & F\end{array}$ $\begin{array}{llllllll}0.7777970141657633 & 0.8888823414043898 & 0.1750651163386081 & F & F & F\end{array}$ $\begin{array}{llllllllll}0.2220037748498481 & 0.1115485555882207 & 0.2655055258448769 & \mathrm{~T} & \mathrm{~T} & \mathrm{~T}\end{array}$ $\begin{array}{lllllllllll}0.5559359712201797 & 0.1105294894759237 & 0.2647633698581331 & \mathrm{~T} & \mathrm{~T} & \mathrm{~T}\end{array}$ $\begin{array}{lllllllllll}0.8896705835683237 & 0.1103214467638186 & 0.2643874871623338 & \text { T } & \text { T } & \text { T }\end{array}$ $\begin{array}{lllllll}0.2240601506798727 & 0.44437466689607073 & 0.2662616739022490 & \text { T } & \text { T } & \text { T }\end{array}$ $\begin{array}{lllllll}0.5537033128261247 & 0.4424357038389937 & 0.2652296646644579 & \mathrm{~T} & \mathrm{~T} & \mathrm{~T}\end{array}$ $\begin{array}{llllllllll}0.8887324712713486 & 0.4456885498305745 & 0.2638488297735581 & \mathrm{~T} & \mathrm{~T} & \mathrm{~T}\end{array}$ $\begin{array}{lllllllll}0.2225217621389413 & 0.7778236173504700 & 0.2652024980176155 & \mathrm{~T} & \mathrm{~T} & \mathrm{~T}\end{array}$ $\begin{array}{lllllllll}0.5561183791343209 & 0.7780624918752694 & 0.2655986524347366 & \mathrm{~T} & \mathrm{~T} & \mathrm{~T}\end{array}$ $\begin{array}{lllllll}0.8890649005681943 & 0.7789154017149545 & 0.2650028063374269 & \mathrm{~T} & \mathrm{~T} & \mathrm{~T}\end{array}$ $\begin{array}{lllllll}0.0058164709772389 & 0.9967811440035191 & 0.3521955464459429 & \text { T } & \text { T } & \text { T }\end{array}$ $\begin{array}{llllllll}0.3345407033212670 & 0.9994495039772926 & 0.3491452754586903 & \text { T } & \text { T } & \text { T }\end{array}$ $\begin{array}{llllllll}0.6638131432238086 & 0.0020706430029036 & 0.3515969818100077 & \mathrm{~T} & \mathrm{~T} & \mathrm{~T}\end{array}$ $\begin{array}{llllllllll}0.9972523154339522 & 0.3386025702753698 & 0.3491402376436824 & \mathrm{~T} & \mathrm{~T} & \mathrm{~T}\end{array}$ $\begin{array}{llllllll}0.3410862098954021 & 0.3345962407368051 & 0.3534016843204610 & \mathrm{~T} & \mathrm{~T} & \mathrm{~T}\end{array}$ $\begin{array}{llllllll}0.6682905611205875 & 0.3326350853795056 & 0.3483491293983694 & \mathrm{~T} & \mathrm{~T} & \mathrm{~T}\end{array}$ $\begin{array}{llllllllll}0.0018466799462907 & 0.6666606181912436 & 0.3497773171446861 & \mathrm{~T} & \mathrm{~T} & \mathrm{~T}\end{array}$ $\begin{array}{llllllllllll}0.3356023962023739 & 0.6703598678329384 & 0.3530687829710056 & \mathrm{~T} & \mathrm{~T} & \mathrm{~T}\end{array}$ $\begin{array}{llllllllll}0.6646295371270764 & 0.6572586630986715 & 0.3488948820258068 & \mathrm{~T} & \mathrm{~T} & \mathrm{~T}\end{array}$ $\begin{array}{llllllllll}0.4528424115074486 & 0.5621723341482251 & 0.4106274121490990 & \mathrm{~T} & \mathrm{~T} & \mathrm{~T}\end{array}$ $\begin{array}{lllllllll}0.1458991291032975 & 0.4395291248821114 & 0.4579951984285337 & \mathrm{~T} & \mathrm{~T} & \mathrm{~T}\end{array}$ $\begin{array}{llllllll}0.7576841110188770 & 0.7906985023430286 & 0.4575405778137371 & \mathrm{~T} & \mathrm{~T} & \mathrm{~T}\end{array}$ $\begin{array}{lllllllll}0.8951752451396521 & 0.1178376212114946 & 0.3969377749626274 & \mathrm{~T} & \mathrm{~T} & \mathrm{~T}\end{array}$

28) $\mathrm{C}-\mathrm{Co}(211)-1 \times 3$

$\operatorname{Co}(211)-3 \times 3$ 1.00000000000000 6.0968000000000000 0.0000000000000000 0.0000000000000000

$\begin{array}{lll}0.0000000000000000 & 0.0000000000000000\end{array}$ $\begin{array}{ll}7.4669999999999996 & 0.0000000000000000\end{array}$ $0.0000000000000000 \quad 23.6221999999999994$ 
Co C

$48 \quad 1$

Selective dynamics

Direct

$\begin{array}{lllllllll}0.0039768403096687 & 0.1666663988214836 & 0.9745822997011260 & F & F & F\end{array}$ $\begin{array}{llllllll}0.0039765122687285 & 0.5000000000000000 & 0.9745819610366553 & F & F & F\end{array}$ $\begin{array}{lllllllll}0.0039768403096687 & 0.8333336011785164 & 0.9745822997011260 & F & F & F\end{array}$ $\begin{array}{lllllllll}0.3315888662905095 & 0.0000000000000000 & 0.0034278771663949 & F & F & F\end{array}$ $\begin{array}{llllllll}0.3315855858811148 & 0.3333350743270387 & 0.0034291471581795 & F & F & F\end{array}$ $\begin{array}{llllllll}0.3315855858811148 & 0.6666647917503710 & 0.0034291471581795 & F & F & F\end{array}$ $\begin{array}{lllllllll}0.6664853693741009 & 0.1666655952859273 & 0.0285661792720404 & F & F & F\end{array}$ $\begin{array}{lllllllll}0.6664884857630256 & 0.5000000000000000 & 0.0285654596100287 & F & F & F\end{array}$ $\begin{array}{llllllll}0.6664853693741009 & 0.8333344047140727 & 0.0285661792720404 & F & F & F\end{array}$ $\begin{array}{llllllll}0.9983825941477491 & 0.0000000000000000 & 0.0609119811025209 & F & F & F\end{array}$ $\begin{array}{llllllll}0.9983791497178842 & 0.3333345386366702 & 0.0609079171288016 & F & F & F\end{array}$ $\begin{array}{llllllll}0.9983791497178842 & 0.6666654613633298 & 0.0609079171288016 & F & F & F\end{array}$ $\begin{array}{lllllllll}0.3314264860254568 & 0.1666650595955517 & 0.0918800111759310 & F & F & F\end{array}$ $\begin{array}{lllllllll}0.3314281262301506 & 0.5000000000000000 & 0.0918805615057039 & F & F & F\end{array}$ $\begin{array}{llllllll}0.3314264860254568 & 0.8333349404044483 & 0.0918800111759310 & F & F & F\end{array}$ $\begin{array}{lllllllll}0.6654751673008761 & 0.0000000000000000 & 0.1205095207050988 & F & F & F\end{array}$ $\begin{array}{llllllll}0.6654631938065876 & 0.3333330654881479 & 0.1205040597404121 & F & F & F\end{array}$ $\begin{array}{llllllll}0.6654631938065876 & 0.6666669345118521 & 0.1205040597404121 & F & F & F\end{array}$ $\begin{array}{llllllll}0.0009121178323070 & 0.1666669345118521 & 0.1526111031148645 & F & F & F\end{array}$ $\begin{array}{llllllll}0.0009114617504267 & 0.5000000000000000 & 0.1526112301140472 & F & F & F\end{array}$ $\begin{array}{lllllllll}0.0009121178323070 & 0.8333330654881479 & 0.1526111031148645 & F & F & F\end{array}$ $\begin{array}{lllllllll}0.3340214538774404 & 0.0000000000000000 & 0.1833969740329024 & F & F & F\end{array}$ $\begin{array}{lllllllll}0.3340229300616713 & 0.3333327976429601 & 0.1833968470337197 & F & F & F\end{array}$ $\begin{array}{llllllllll}0.3340229300616713 & 0.6666672023570399 & 0.1833968470337197 & F & F & F\end{array}$ $\begin{array}{llllllll}0.6651836834864367 & 0.1670241756699727 & 0.2121787557005698 & \mathrm{~T} & \mathrm{~T} & \mathrm{~T}\end{array}$ $\begin{array}{llllllll}0.6653551290437728 & 0.5000130881130070 & 0.2120218271879724 & \mathrm{~T} & \mathrm{~T} & \mathrm{~T}\end{array}$ $\begin{array}{lllllll}0.6651900106739954 & 0.8329630275822837 & 0.2121744677031792 & \mathrm{~T} & \mathrm{~T} & \mathrm{~T}\end{array}$ $\begin{array}{llllllll}0.9972328080415268 & 0.0000066152270395 & 0.2425129308967935 & \mathrm{~T} & \mathrm{~T} & \mathrm{~T}\end{array}$ $\begin{array}{lllllll}0.9983297269388163 & 0.3341507892971681 & 0.2427182805003978 & \mathrm{~T} & \mathrm{~T} & \mathrm{~T}\end{array}$ $\begin{array}{lllllll}0.9983315371876574 & 0.6658341425011695 & 0.2427143291968946 & \mathrm{~T} & \mathrm{~T} & \mathrm{~T}\end{array}$ $\begin{array}{llllllll}0.3324577800673333 & 0.1664097601781237 & 0.2747655903034971 & \mathrm{~T} & \mathrm{~T} & \mathrm{~T}\end{array}$ $\begin{array}{llllllll}0.3331241199194607 & 0.4999919888602291 & 0.2744422805143385 & \mathrm{~T} & \mathrm{~T} & \mathrm{~T}\end{array}$ $\begin{array}{llllllll}0.3324810389836140 & 0.8335852469511348 & 0.2747659202661329 & \mathrm{~T} & \mathrm{~T} & \mathrm{~T}\end{array}$ $\begin{array}{lllllll}0.6643907520026215 & 0.0000068255952465 & 0.3039697132717603 & \mathrm{~T} & \mathrm{~T} & \mathrm{~T}\end{array}$ $\begin{array}{llllllll}0.6672905079228441 & 0.3339344911607027 & 0.3033625663218367 & \mathrm{~T} & \mathrm{~T} & \mathrm{~T}\end{array}$ $\begin{array}{lllllll}0.6672824856403432 & 0.6660727410027502 & 0.3033569694191066 & \mathrm{~T} & \mathrm{~T} & \mathrm{~T}\end{array}$ $\begin{array}{lllllll}-0.0026704406428508 & 0.1654753587475361 & 0.3330968920647463 & \mathrm{~T} & \mathrm{~T} & \mathrm{~T}\end{array}$ $\begin{array}{lllllllll}0.0002328669983543 & 0.4999894400047976 & 0.3346721302241367 & \mathrm{~T} & \mathrm{~T} & \mathrm{~T}\end{array}$ $\begin{array}{lllllll}-0.0026720503707607 & 0.8345075545028593 & 0.3330944240998204 & \mathrm{~T} & \mathrm{~T} & \mathrm{~T}\end{array}$ $\begin{array}{lllllll}0.3278840689635764 & -0.0000003774673127 & 0.3675056990710739 & \mathrm{~T} & \mathrm{~T} & \mathrm{~T}\end{array}$ $\begin{array}{lllllll}0.3341810212159383 & 0.3332111924702934 & 0.3650289057677424 & \mathrm{~T} & \mathrm{~T} & \mathrm{~T}\end{array}$ $\begin{array}{llllllll}0.3341551568536060 & 0.6667969758752127 & 0.3650343529446300 & \mathrm{~T} & \mathrm{~T} & \mathrm{~T}\end{array}$ $\begin{array}{lllllll}0.6648317572615734 & 0.1654293860992728 & 0.3915219353401320 & \mathrm{~T} & \mathrm{~T} & \mathrm{~T}\end{array}$ $\begin{array}{llllllll}0.6604604430964073 & 0.4999781943597921 & 0.3938993571935223 & \mathrm{~T} & \mathrm{~T} & \mathrm{~T}\end{array}$ $\begin{array}{lllllllll}0.6648061086327516 & 0.8345536393561751 & 0.3915132344610947 & \mathrm{~T} & \mathrm{~T} & \mathrm{~T}\end{array}$ $\begin{array}{lllllll}0.9862308419131331 & -0.0000215381943695 & 0.4196414186557375 & \mathrm{~T} & \mathrm{~T} & \mathrm{~T}\end{array}$ $\begin{array}{llllllll}0.0132257522051061 & 0.3201874704431405 & 0.4223733261680466 & \mathrm{~T} & \mathrm{~T} & \mathrm{~T}\end{array}$ $\begin{array}{lllllll}0.0131448589671364 & 0.6797592893502391 & 0.4223662268661467 & \mathrm{~T} & \mathrm{~T} & \mathrm{~T}\end{array}$ $\begin{array}{lllllll}0.8577264256830258 & 0.4999465704763640 & 0.4500626887079267 & \mathrm{~T} & \mathrm{~T} & \mathrm{~T}\end{array}$ 


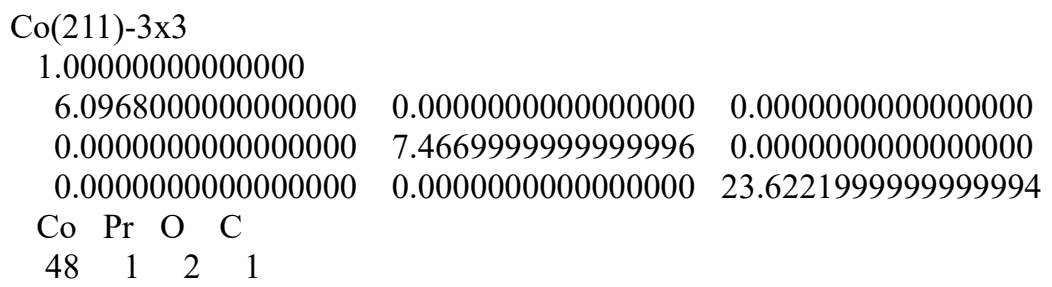

Selective dynamics

Direct

$\begin{array}{llllllll}0.0039768403096687 & 0.1666663988214765 & 0.9745822997011331 & F & F & F\end{array}$ $\begin{array}{llllllll}0.0039765122687285 & 0.50000000000000000 & 0.9745822997011331 & F & F & F\end{array}$ $\begin{array}{llllllll}0.0039768403096687 & 0.8333336011785235 & 0.9745822997011331 & F & F & F\end{array}$ $\begin{array}{lllllllll}0.3315888662905095 & 0.0000000000000000 & 0.0034278771663878 & \mathrm{~F} & \mathrm{~F} & \mathrm{~F}\end{array}$ $\begin{array}{llllllll}0.3315855858811219 & 0.3333350743270387 & 0.0034291471581795 & F & F & F\end{array}$ $\begin{array}{llllllll}0.3315855858811219 & 0.6666647917503710 & 0.0034291471581795 & F & F & F\end{array}$ $\begin{array}{lllllllll}0.6664853693741009 & 0.1666655952859273 & 0.0285661792720404 & F & F & F\end{array}$ $\begin{array}{llllllll}0.6664884857630327 & 0.50000000000000000 & 0.0285654596100287 & F & F & F\end{array}$ $\begin{array}{llllllll}0.6664853693741009 & 0.8333344047140727 & 0.0285661792720404 & F & F & F\end{array}$ $\begin{array}{llllllll}0.9983825941477491 & 0.0000000000000000 & 0.0609119811025209 & F & F & F\end{array}$ $\begin{array}{llllllll}0.9983791497178913 & 0.3333345386366702 & 0.0609079171288016 & F & F & F\end{array}$ $\begin{array}{lllllllll}0.9983791497178913 & 0.6666654613633298 & 0.0609079171288016 & F & F & F\end{array}$ $\begin{array}{lllllll}0.3314264860254568 & 0.1666650595955517 & 0.0918800111759310 & F & F & F\end{array}$ $\begin{array}{lllllll}0.3314281262301506 & 0.5000000000000000 & 0.0918805615056968 & F & F & F\end{array}$ $\begin{array}{llllllll}0.3314264860254568 & 0.8333349404044483 & 0.0918800111759310 & F & F & F\end{array}$ $\begin{array}{lllllll}0.6654751673008832 & 0.0000000000000000 & 0.1205095207050988 & F & F & F\end{array}$ $\begin{array}{llllllll}0.6654631938065876 & 0.3333330654881479 & 0.1205040597404121 & F & F & F\end{array}$ $\begin{array}{lllllll}0.6654631938065876 & 0.6666669345118521 & 0.1205040597404121 & F & F & F\end{array}$ $\begin{array}{lllllllll}0.0009121178323070 & 0.1666669345118521 & 0.1526111031148716 & F & F & F\end{array}$ $\begin{array}{lllllll}0.0009114617504267 & 0.5000000000000000 & 0.1526112301140472 & F & F & F\end{array}$ $\begin{array}{lllllll}0.0009121178323070 & 0.8333330654881479 & 0.1526111031148716 & F & F & F\end{array}$ $\begin{array}{llllllll}0.3340214538774404 & 0.0000000000000000 & 0.1833969740329024 & F & F & F\end{array}$ $\begin{array}{lllllllll}0.3340229300616713 & 0.3333327976429601 & 0.1833968470337197 & F & F & F\end{array}$ $\begin{array}{lllllllll}0.3340229300616713 & 0.6666672023570399 & 0.1833968470337197 & F & F & F\end{array}$ $\begin{array}{llllllll}0.6646579422798129 & 0.1664127234338406 & 0.2117747334176793 & \mathrm{~T} & \mathrm{~T} & \mathrm{~T}\end{array}$ $\begin{array}{lllllllll}0.6657979986372220 & 0.4997842039222730 & 0.2119388020351741 & \mathrm{~T} & \mathrm{~T} & \mathrm{~T}\end{array}$ $\begin{array}{llllllll}0.6657221584832392 & 0.8340930578824738 & 0.2118341186016801 & \mathrm{~T} & \mathrm{~T} & \mathrm{~T}\end{array}$ $\begin{array}{lllllllll}0.9968876559786963 & -0.0000544936298271 & 0.2432890268429820 & \text { T } & \text { T } & \text { T }\end{array}$ $\begin{array}{lllllll}-0.0019255420666949 & 0.3339311181973192 & 0.2433260902881982 & \mathrm{~T} & \mathrm{~T} & \mathrm{~T}\end{array}$ $\begin{array}{llllllllll}0.0003481260475894 & 0.6665824410356554 & 0.2429495377698467 & \mathrm{~T} & \mathrm{~T} & \mathrm{~T}\end{array}$ $\begin{array}{lllllllllll}0.3317136711743734 & 0.1671248175961575 & 0.2755390373540217 & \mathrm{~T} & \mathrm{~T} & \mathrm{~T}\end{array}$ $\begin{array}{llllllll}0.3345576999534116 & 0.4981509905998547 & 0.2754092151077924 & \mathrm{~T} & \mathrm{~T} & \mathrm{~T}\end{array}$ $\begin{array}{llllllllll}0.3315810143970591 & 0.8339799110526186 & 0.2737337162740914 & \mathrm{~T} & \mathrm{~T} & \mathrm{~T}\end{array}$ $\begin{array}{lllllll}0.6648905315398171 & 0.0018473417868035 & 0.3030084900945898 & \mathrm{~T} & \mathrm{~T} & \mathrm{~T}\end{array}$ $\begin{array}{lllllll}0.6669167571740654 & 0.3296021255006357 & 0.3023121363358954 & \mathrm{~T} & \mathrm{~T} & \mathrm{~T}\end{array}$ $\begin{array}{llllllll}0.6655731368409288 & 0.6674684992350458 & 0.3020357481165770 & \mathrm{~T} & \mathrm{~T} & \mathrm{~T}\end{array}$ $\begin{array}{llllllll}0.9964315355195292 & 0.1684671024551909 & 0.3342324829624961 & \mathrm{~T} & \mathrm{~T} & \mathrm{~T}\end{array}$ $\begin{array}{llllllll}0.0033513996031114 & 0.5001131019420725 & 0.3349380697803835 & \mathrm{~T} & \mathrm{~T} & \mathrm{~T}\end{array}$ $\begin{array}{llllllll}0.9966926634306925 & 0.8321960938931868 & 0.3350348849175179 & \mathrm{~T} & \mathrm{~T} & \mathrm{~T}\end{array}$ $\begin{array}{lllllllll}0.3271289452940551 & 0.9956131632329416 & 0.3648450865122700 & \mathrm{~T} & \mathrm{~T} & \mathrm{~T}\end{array}$ $\begin{array}{lllllll}0.3265666387023504 & 0.3304931924421498 & 0.3696424071447743 & \mathrm{~T} & \mathrm{~T} & \mathrm{~T}\end{array}$ $\begin{array}{llllllll}0.3248105943413145 & 0.6724509135259389 & 0.3636382600404359 & \mathrm{~T} & \mathrm{~T} & \mathrm{~T}\end{array}$ $\begin{array}{lllllll}0.6686430754909513 & 0.1638154590033586 & 0.3957732472969617 & \mathrm{~T} & \mathrm{~T} & \mathrm{~T}\end{array}$ $\begin{array}{llllllll}0.6714961208660476 & 0.4964449535058141 & 0.3865363404952531 & \mathrm{~T} & \mathrm{~T} & \mathrm{~T}\end{array}$ 
$\begin{array}{lllllll}0.6529073982275546 & 0.8359636206798809 & 0.3911859104607936 & \mathrm{~T} & \mathrm{~T} & \mathrm{~T}\end{array}$ $\begin{array}{lllllllll}0.9979768015573773 & 0.0065381239752620 & 0.4223591589198578 & \mathrm{~T} & \mathrm{~T} & \mathrm{~T}\end{array}$ $\begin{array}{lllllllll}0.0019643069072233 & 0.3321168766854257 & 0.4259412802248017 & \mathrm{~T} & \mathrm{~T} & \mathrm{~T}\end{array}$ $\begin{array}{lllllllllll}0.9799101016835695 & 0.6641205640235570 & 0.4296190706180286 & \mathrm{~T} & \mathrm{~T} & \mathrm{~T}\end{array}$ $\begin{array}{llllllllll}0.4224615086570892 & 0.6015398808323551 & 0.4824064236727929 & \mathrm{~T} & \mathrm{~T} & \mathrm{~T}\end{array}$ $\begin{array}{llllllll}0.5193008568252225 & 0.3429196448161501 & 0.4413762011409862 & \mathrm{~T} & \mathrm{~T} & \mathrm{~T}\end{array}$ $\begin{array}{llllllll}0.0764276318399706 & 0.4938548935519562 & 0.4835555458943468 & \mathrm{~T} & \mathrm{~T} & \mathrm{~T}\end{array}$ $\begin{array}{llllllllll}0.8143935753273973 & 0.8451092670500731 & 0.4545985771968322 & \mathrm{~T} & \mathrm{~T} & \mathrm{~T}\end{array}$

30) $\mathrm{C}-\mathrm{Na}_{2} \mathrm{O}-\mathrm{Co}(211)-1 \times 3$

$\operatorname{Co}(211)-3 \times 3$ 1.00000000000000 $\begin{array}{llll}6.0968000000000000 & 0.0000000000000000 & 0.0000000000000000\end{array}$ $\begin{array}{llll}0.0000000000000000 & 7.46699999999999996 & 0.0000000000000000\end{array}$ $\begin{array}{llll}0.0000000000000000 & 0.0000000000000000 & 23.6221999999999994\end{array}$

Co $\mathrm{O} \quad \mathrm{Na} \mathrm{C}$

$\begin{array}{llll}48 & 1 & 2 & 1\end{array}$

Selective dynamics

Direct

$\begin{array}{llllllll}0.0039768403096687 & 0.1666663988214765 & 0.9745822997011331 & \mathrm{~F} & \mathrm{~F} & \mathrm{~F}\end{array}$ $\begin{array}{llllllllll}0.0039765122687285 & 0.5000000000000000 & 0.9745822997011331 & F & F & F\end{array}$ $\begin{array}{llllllll}0.0039768403096687 & 0.8333336011785235 & 0.9745822997011331 & F & F & F\end{array}$ $\begin{array}{llllllll}0.3315888662905095 & 0.0000000000000000 & 0.0034278771663878 & F & F & F\end{array}$ $\begin{array}{llllllll}0.3315855858811219 & 0.3333350743270387 & 0.0034291471581795 & F & F & F\end{array}$ $\begin{array}{llllllll}0.3315855858811219 & 0.6666647917503710 & 0.0034291471581795 & \mathrm{~F} & \mathrm{~F} & \mathrm{~F}\end{array}$ $\begin{array}{lllllllll}0.6664853693741009 & 0.1666655952859273 & 0.0285661792720404 & F & F & F\end{array}$ $\begin{array}{lllllllll}0.6664884857630327 & 0.5000000000000000 & 0.0285654596100287 & \mathrm{~F} & \mathrm{~F} & \mathrm{~F}\end{array}$ $\begin{array}{llllllllll}0.6664853693741009 & 0.8333344047140727 & 0.0285661792720404 & F & F & F\end{array}$ $\begin{array}{llllllll}0.9983825941477491 & 0.0000000000000000 & 0.0609119811025209 & F & F & F\end{array}$ $\begin{array}{lllllll}0.9983791497178913 & 0.3333345386366702 & 0.0609079171288016 & F & F & F\end{array}$ $\begin{array}{llllllllll}0.9983791497178913 & 0.6666654613633298 & 0.0609079171288016 & F & F & F\end{array}$ $\begin{array}{lllllllllll}0.3314264860254568 & 0.1666650595955517 & 0.0918800111759310 & \mathrm{~F} & \mathrm{~F} & \mathrm{~F}\end{array}$ $\begin{array}{lllllllll}0.3314281262301506 & 0.5000000000000000 & 0.0918805615056968 & \mathrm{~F} & \mathrm{~F} & \mathrm{~F}\end{array}$ $\begin{array}{llllllllll}0.3314264860254568 & 0.8333349404044483 & 0.0918800111759310 & \mathrm{~F} & \mathrm{~F} & \mathrm{~F}\end{array}$ $\begin{array}{llllllll}0.6654751673008832 & 0.0000000000000000 & 0.1205095207050988 & F & F & F\end{array}$ $\begin{array}{lllllllll}0.6654631938065876 & 0.3333330654881479 & 0.1205040597404121 & F & F & F\end{array}$ $\begin{array}{llllllllll}0.6654631938065876 & 0.6666669345118521 & 0.1205040597404121 & F & F & F\end{array}$ $\begin{array}{lllllll}0.0009121178323070 & 0.1666669345118521 & 0.1526111031148716 & F & F & F\end{array}$ $\begin{array}{llllllll}0.0009114617504267 & 0.5000000000000000 & 0.1526112301140472 & F & F & F\end{array}$ $\begin{array}{llllllll}0.0009121178323070 & 0.8333330654881479 & 0.1526111031148716 & F & F & F\end{array}$ $\begin{array}{llllllllll}0.3340214538774404 & 0.0000000000000000 & 0.1833969740329024 & F & F & F\end{array}$ $\begin{array}{lllllllllll}0.3340229300616713 & 0.3333327976429601 & 0.1833968470337197 & \mathrm{~F} & \mathrm{~F} & \mathrm{~F}\end{array}$ $\begin{array}{llllllll}0.3340229300616713 & 0.6666672023570399 & 0.1833968470337197 & F & F & F\end{array}$ $\begin{array}{lllllllllll}0.6660229830382043 & 0.1664180394931851 & 0.2118080413153266 & \mathrm{~T} & \mathrm{~T} & \mathrm{~T}\end{array}$ $\begin{array}{lllllllll}0.6664426563819583 & 0.5004865387322640 & 0.2118496763119750 & \mathrm{~T} & \mathrm{~T} & \mathrm{~T}\end{array}$ $\begin{array}{llllllllll}0.6650819728261590 & 0.8329395921601445 & 0.2118653610911357 & \mathrm{~T} & \mathrm{~T} & \mathrm{~T}\end{array}$ $\begin{array}{llllllllll}0.9982951932438143 & 0.9994225565787256 & 0.2432422663639840 & \mathrm{~T} & \mathrm{~T} & \mathrm{~T}\end{array}$ $\begin{array}{llllllll}0.9984396644800451 & 0.3340788787620101 & 0.2433175370757806 & \mathrm{~T} & \mathrm{~T} & \mathrm{~T}\end{array}$ $\begin{array}{llllllll}0.9990398098020749 & 0.6674718252495289 & 0.2435036232656308 & \mathrm{~T} & \mathrm{~T} & \mathrm{~T}\end{array}$ $\begin{array}{lllllllll}0.3330188721966958 & 0.1656560887983264 & 0.2744756479918403 & \mathrm{~T} & \mathrm{~T} & \mathrm{~T}\end{array}$ $\begin{array}{llllllllll}0.3321828815277689 & 0.5003364737550637 & 0.2741907694773036 & \mathrm{~T} & \mathrm{~T} & \mathrm{~T}\end{array}$ $\begin{array}{llllllllll}0.3317471108629577 & 0.8343049750536432 & 0.2747477952110169 & \mathrm{~T} & \mathrm{~T} & \mathrm{~T}\end{array}$ 
$\begin{array}{lllllll}0.6662758850264000 & 0.9996581887327556 & 0.3026024407681248 & \mathrm{~T} & \mathrm{~T} & \mathrm{~T}\end{array}$ $\begin{array}{llllllllll}0.6654621338386121 & 0.3335342855802528 & 0.3027331869064074 & \mathrm{~T} & \mathrm{~T} & \mathrm{~T}\end{array}$ $\begin{array}{lllllllllll}0.6652426871261138 & 0.6671541227364572 & 0.3032493960256671 & \mathrm{~T} & \mathrm{~T} & \mathrm{~T}\end{array}$ $\begin{array}{llllllllll}0.9982388363238468 & 0.1675822064598327 & 0.3336601089753727 & \mathrm{~T} & \mathrm{~T} & \mathrm{~T}\end{array}$ $\begin{array}{llllllllll}0.9976017636900938 & 0.5015922075961459 & 0.3355140442157161 & \mathrm{~T} & \mathrm{~T} & \mathrm{~T}\end{array}$ $\begin{array}{llllllll}0.9986305562196982 & 0.8340520750107976 & 0.3356327237275910 & \mathrm{~T} & \mathrm{~T} & \mathrm{~T}\end{array}$ $\begin{array}{llllllll}0.3300248634253058 & 0.0002047020037566 & 0.3674997441658528 & \mathrm{~T} & \mathrm{~T} & \mathrm{~T}\end{array}$ $\begin{array}{llllllll}0.3298426701306598 & 0.3311673307645195 & 0.3633138532849471 & \mathrm{~T} & \mathrm{~T} & \mathrm{~T}\end{array}$ $\begin{array}{llllllll}0.3289739607705178 & 0.6670065843298526 & 0.3630568908775860 & \mathrm{~T} & \mathrm{~T} & \mathrm{~T}\end{array}$ $\begin{array}{lllllllll}0.6664791600617851 & 0.1700704161599977 & 0.3898430881548156 & \mathrm{~T} & \mathrm{~T} & \mathrm{~T}\end{array}$ $\begin{array}{lllllllll}0.6616953667926030 & 0.4960781099051799 & 0.3898620023270907 & \mathrm{~T} & \mathrm{~T} & \mathrm{~T}\end{array}$ $\begin{array}{lllllllll}0.6580597562294932 & 0.8358733027627758 & 0.3927417749190200 & \mathrm{~T} & \mathrm{~T} & \mathrm{~T}\end{array}$ $\begin{array}{llllllll}0.9984268947398636 & 0.0142724128821413 & 0.4236781404465580 & \mathrm{~T} & \mathrm{~T} & \mathrm{~T}\end{array}$ $\begin{array}{llllllll}0.9902844231834672 & 0.3346277470016989 & 0.4247344140392910 & \mathrm{~T} & \mathrm{~T} & \mathrm{~T}\end{array}$ $\begin{array}{llllllll}0.9914828848889192 & 0.6578119681192123 & 0.4303955930214121 & \text { T } & \text { T } & \text { T }\end{array}$ $\begin{array}{llllllllll}0.1219335239543362 & 0.4872827295581384 & 0.4764193173124881 & \mathrm{~T} & \mathrm{~T} & \mathrm{~T}\end{array}$ $\begin{array}{llllllllll}0.3923367510265897 & 0.7254957157231614 & 0.4852715219231505 & \mathrm{~T} & \mathrm{~T} & \mathrm{~T}\end{array}$ $\begin{array}{llllllllll}0.3826205856606456 & 0.2585279674893396 & 0.4845501869723537 & \mathrm{~T} & \mathrm{~T} & \mathrm{~T}\end{array}$ $\begin{array}{llllllllll}0.8311763562963463 & 0.8427626796994238 & 0.4542526628392116 & \mathrm{~T} & \mathrm{~T} & \mathrm{~T}\end{array}$ 